\title{
EVALUATING THE COMPARABILITY OF ENVIRONMENTAL PRODUCT DECLARATIONS FOR USE AS A DECISION-MAKING TOOL FOR BUILDING DESIGNERS
}

\author{
by
}

\begin{abstract}
Matthew David Canario Gelowitz
Bachelor of Architectural Science,

Ryerson University, Toronto, 2014
\end{abstract}

\author{
A thesis \\ presented to Ryerson University \\ in partial fulfillment of the \\ requirements for the degree of \\ Master of Applied Science \\ in the Program of \\ Building Science
}

Toronto, Ontario, Canada, 2016

(c) Matthew Gelowitz 2016 


\section{Author's Declaration for Electronic Submission of a Thesis}

I hereby declare that I am the sole author of this thesis.

I authorize Ryerson University to lend this thesis to other institutions or individuals for the purpose of scholarly research

I further authorize Ryerson University to reproduce this thesis by photocopying or by other means, in total or in part, at the request of other institutions of individuals for the purpose of scholarly research.

I understand that my thesis may be made electronically available to the public. 
EVALUATING THE EFFECTIVENESS OF ENVIRONMENTAL PRODUCT DECLARATIONS AS A DECISION-MAKING TOOL FOR BUILDING DESIGNERS

\author{
by \\ Matthew David Canario Gelowitz \\ Master of Applied Science in Building Science, 2016 \\ Ryerson University, Toronto
}

\begin{abstract}
With its inclusion to LEED®, use of EPDs in the construction industry will accelerate over time. This research examines current practices surrounding the use of EPDs in construction and addresses a key need arising from a case study completed on the first Canadian project to use EPDs. Findings suggest that lack of comparability between claims hinders the ability for them to be used as true, decisive comparative tools on projects. This informed the development of a semi-automated comparison tool for EPDs and PCRs. Three separate construction product categories were chosen for comparison to develop this tool: insulation, flooring, and cladding systems. Comparability was more evident in categories that have had early involvement in publishing environmental claims (such as flooring, because of its human health implications). However, there was concerning evidence regarding the comparability of EPDs that were comparable according to international standards were incomparable according to the comparison framework developed.
\end{abstract}




\section{Acknowledgements}

I would like to extend my sincere gratitude to Professor Jenn McArthur at Ryerson University for her continuous guidance throughout this research process, along with deepening my understanding of building science research. I would also like to thank Professors Dr. Mark Gorgolewski and Vera Straka for their insight throughout.

I wish like to thank the Ontario Centres of Excellence and Julie Scarcella at EcoSpex for funding this project through the VIP1 program, and the financial support received from Ryerson University.

Lastly, thank you to my parents Lyle and Margarita, who have made everything in life possible. Thank you to my family, friends, and loved ones, especially Sahel, who have provided me with their utmost support. 


\section{Contents}

Author's Declaration for Electronic Submission of a Thesis ...............................................

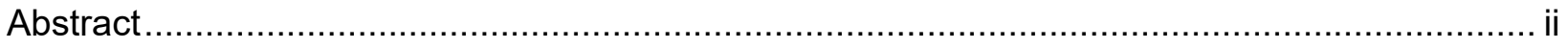

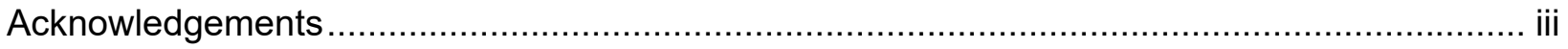

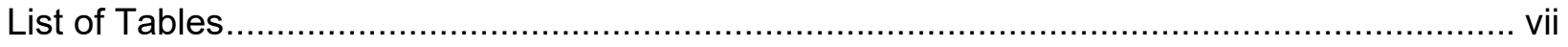

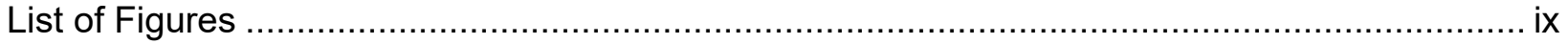

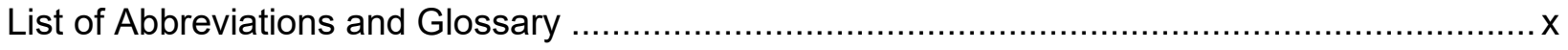

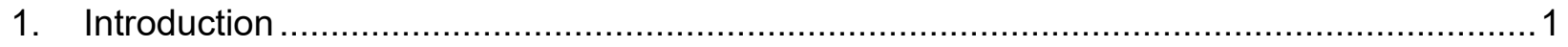

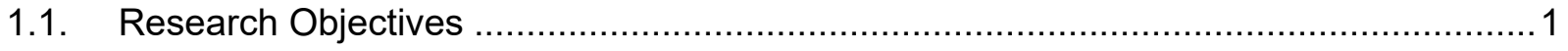

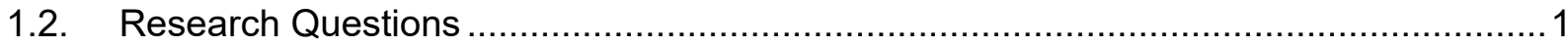

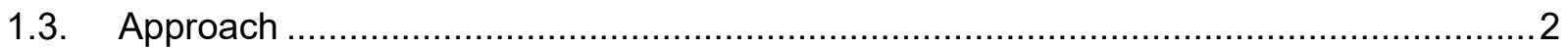

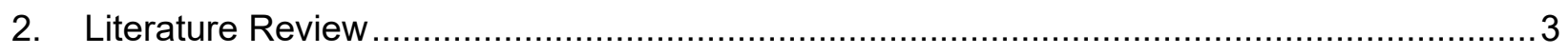

2.1. Key Terms and Definitions: The Simplified Claim Process..................................... 3

2.2. Materials and the Built Environment ........................................................... 3

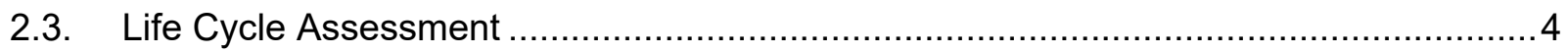

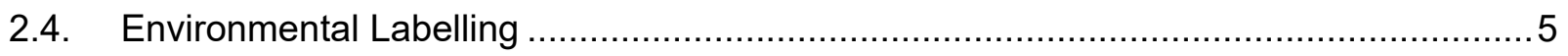

2.5. ISO 14025 and other Associated Standards and Documents ..............................6

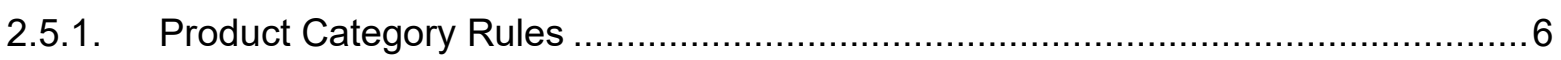

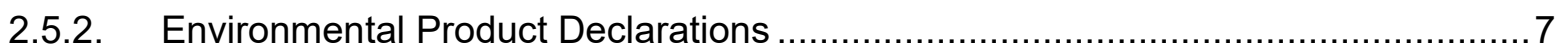

2.6. Drivers for Environmental Product Declaration Use in Construction ............................9

2.7. Environmental Product Declaration Adoption Hindrances........................................ 9

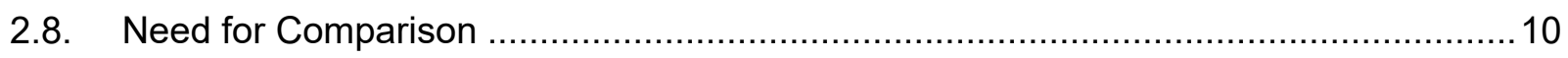

2.8.1. Previous Comparison Work ................................................................... 11

3. Case Study: Canada Green Building Council National Office, Vancouver, BC ..................15

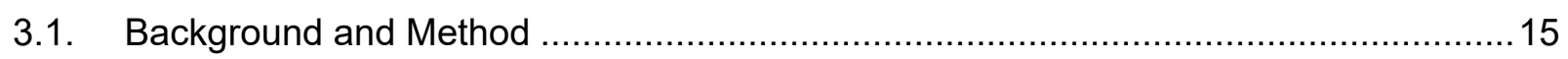

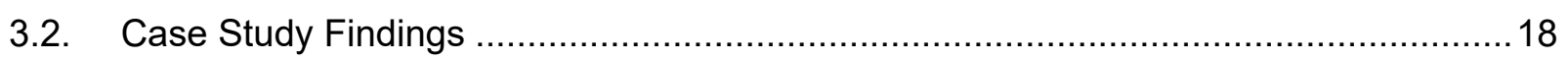

3.2.1. Experience with Environmental Product Declarations.................................... 18

3.2.2. Driving Forces Behind LEED® Credit ..................................................... 18

3.2.3. Impacts of Using Environmental Product Declarations on a Project ....................18

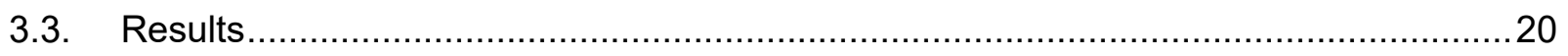

3.3.1. Benefits of Using Environmental Product Declarations on a Project....................20

3.3.2. Drawbacks of Using Environmental Product Declarations on a Project...............20

3.3.3. Concerns with Aspects of Environmental Product Declarations........................21

3.3.4. Willingness to Work with Environmental Product Declarations in the Future ........23 


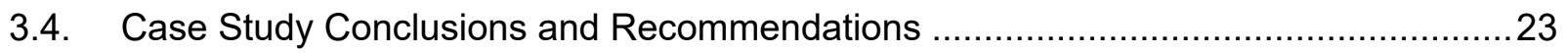

4. Design of the Environmental Product Declaration Comparison Matrix .............................25

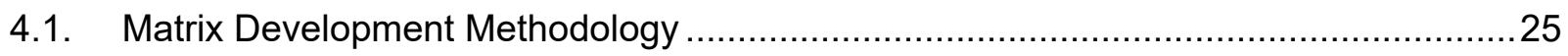

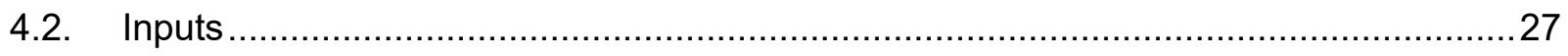

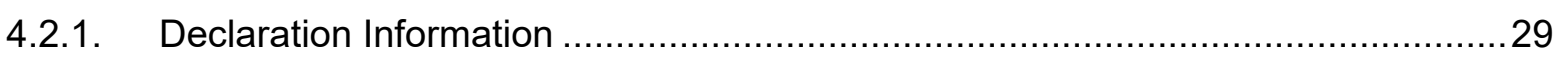

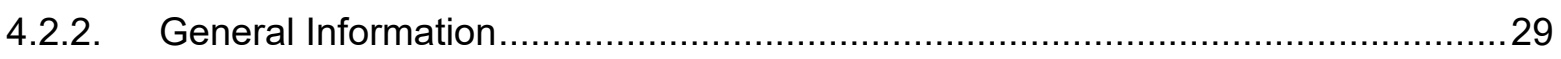

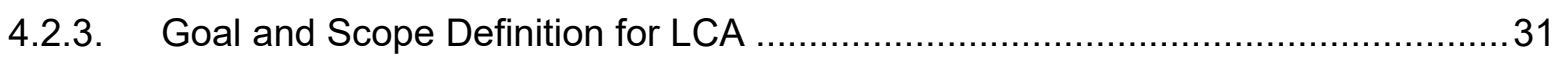

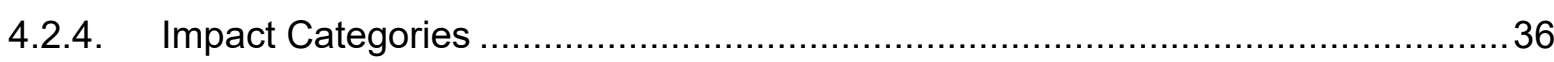

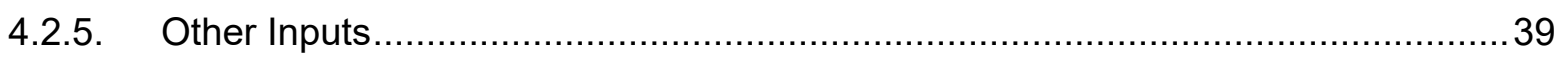

4.3. Environmental Product Declaration Comparison ............................................... 39

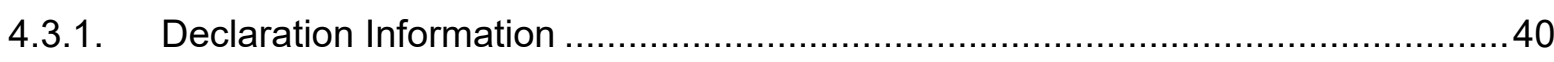

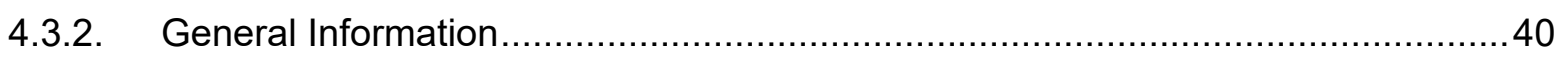

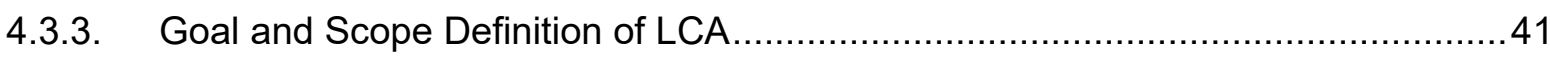

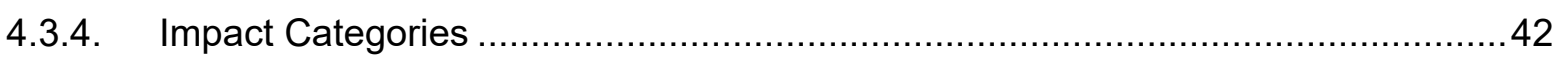

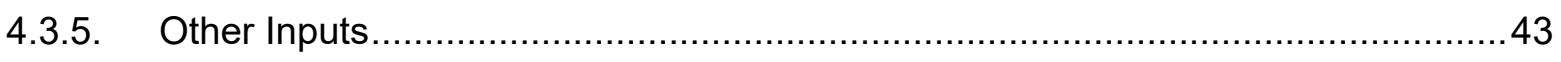

4.4. Environmental Product Declaration Summarization ........................................... 43

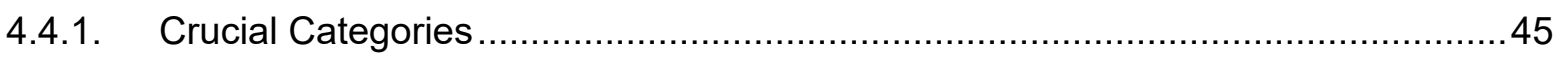

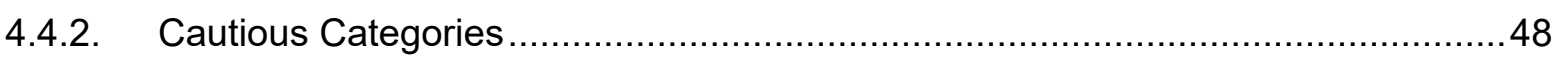

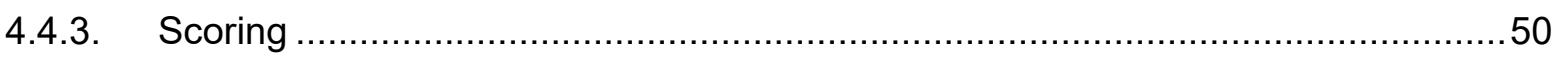

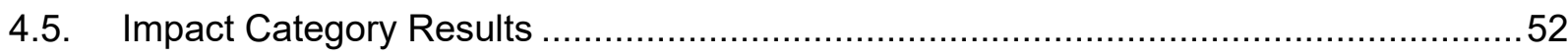

5. Design of the Product Category Rule Comparison Matrix ........................................5

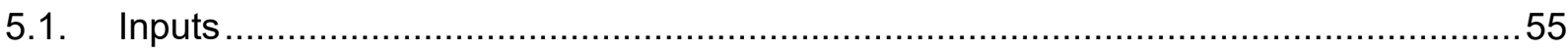

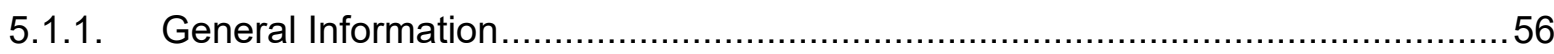

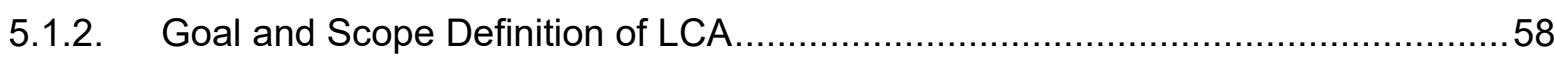

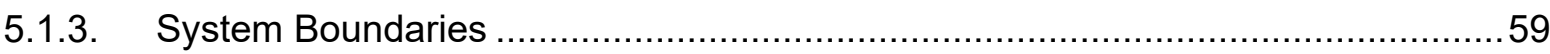

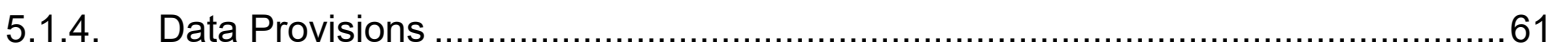

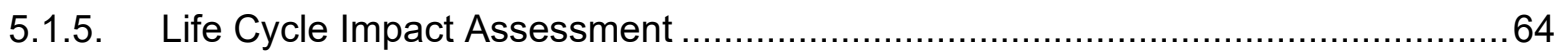

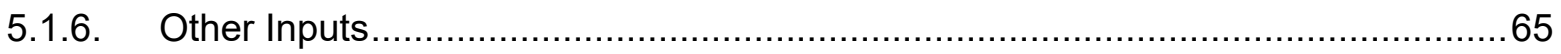

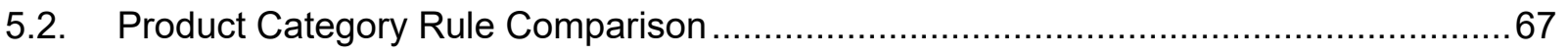

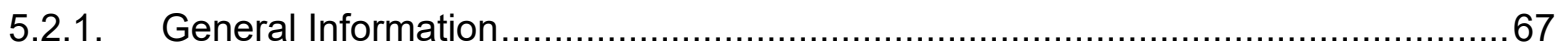

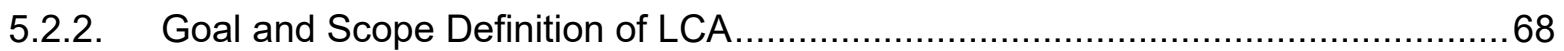

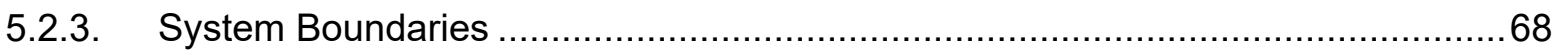

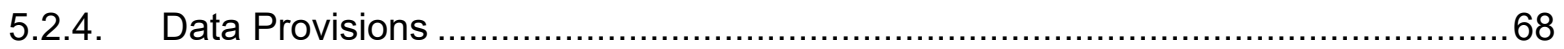

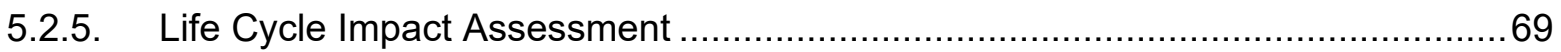

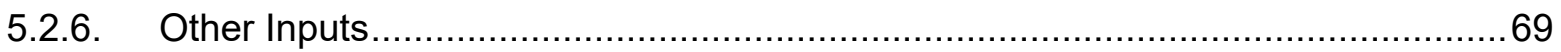




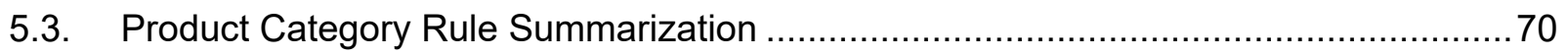

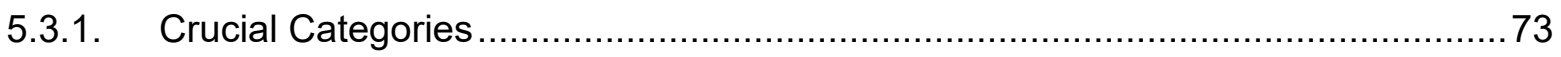

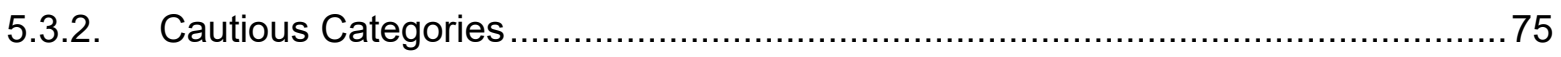

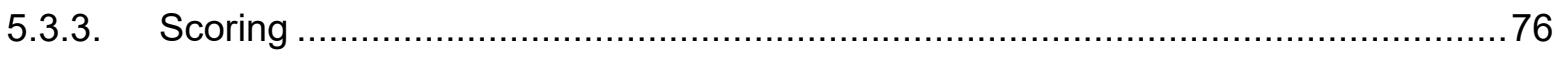

6. Using the Environmental Product Declaration Comparison Matrix ...................................78

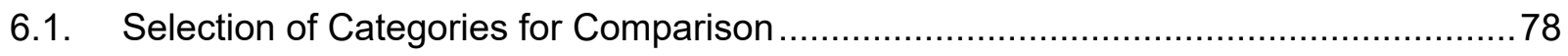

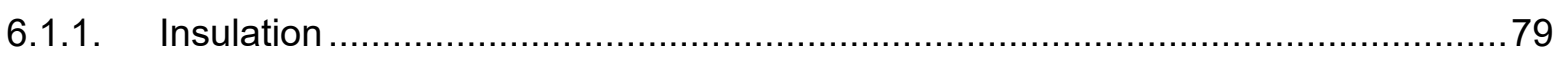

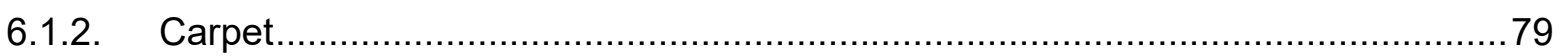

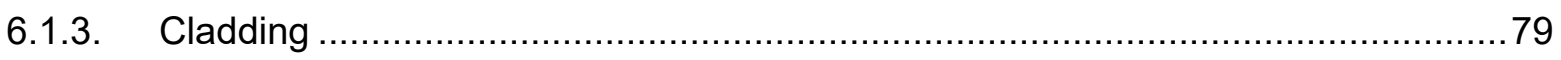

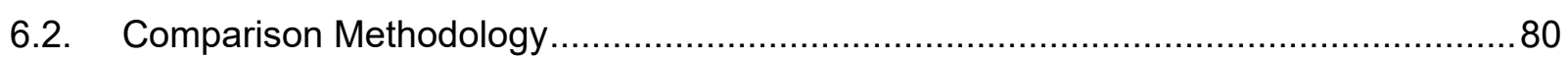

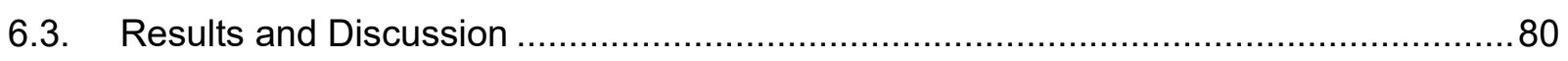

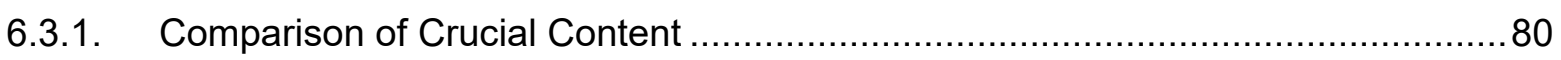

6.3.2. Comparison of Non-Critical Content Requiring Caution ......................................86

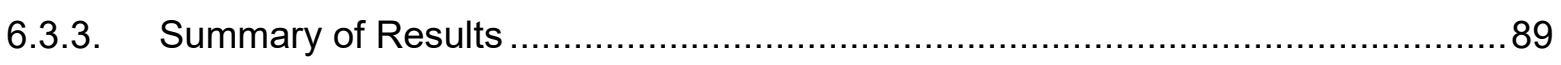

7. Using the Product Category Rule Comparison Matrix ……...........................................92

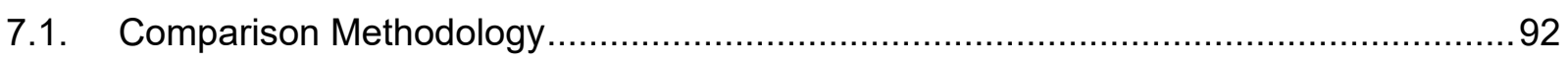

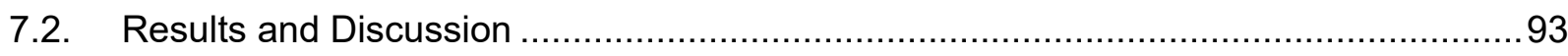

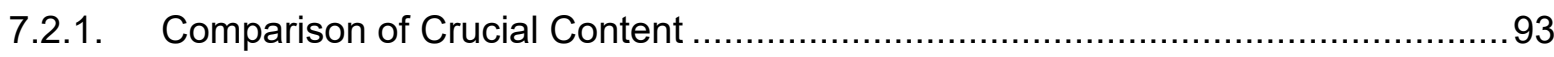

7.2.2. Comparison of Non-Critical Content Requiring Caution ....................................97

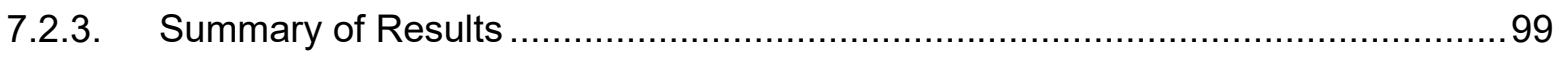

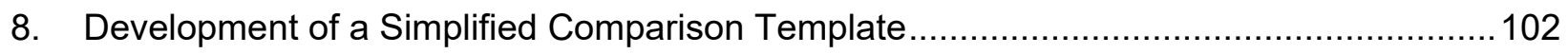

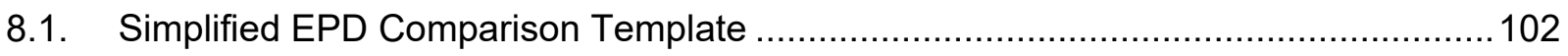

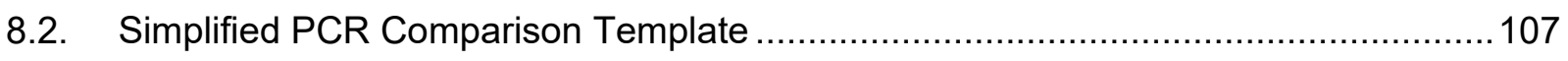

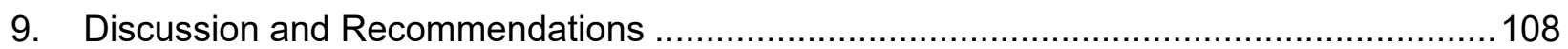

9.1. Issues Associated with Environmental Product Declarations ...................................108

9.2. Issues Associated with Product Category Rules ….............................................113

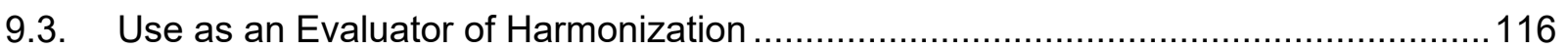

9.4. Suggested Areas of Policy Improvement to Improve Comparability ........................119

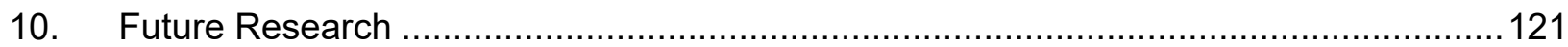

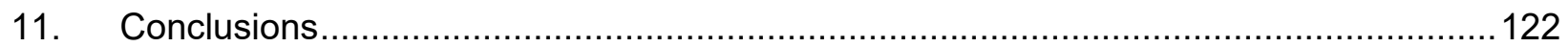

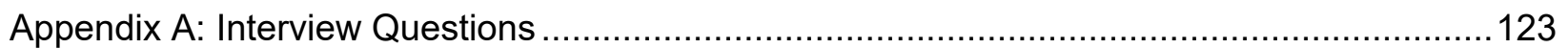

Appendix B: Comparison Matrices, Templates, and Documents Used Within .......................127

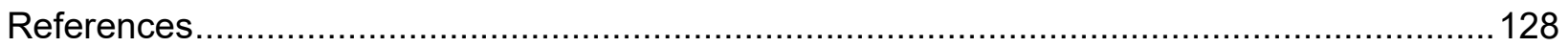




\section{List of Tables}

Table 1: Glossary of EPD-specific technical jargon .....................................................

Table 2: Titles and Definitions of Standards Related to EPDs and PCRs ............................ 8

Table 3: PCR Comparison Template (Subramanian, Ingwersen \& Hensler, 2011)..................12

Table 4: Building Disclosure and Optimization - Environmental Product Declarations Credit

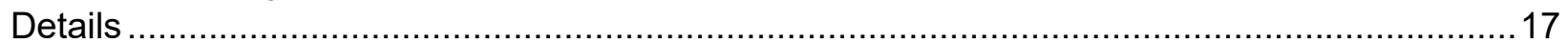

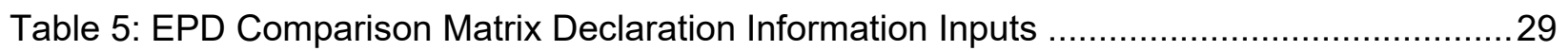

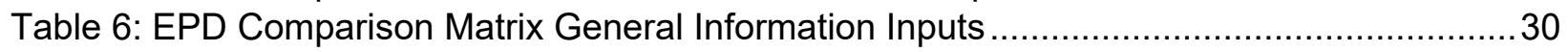

Table 7: Necessary declaration content from ISO 140257.2 with adaptation .........................31

Table 8: EPD Comparison Matrix Functional Unit Inputs...................................................... 32

Table 9: EPD Comparison Matrix System Boundaries First Inputs ........................................ 32

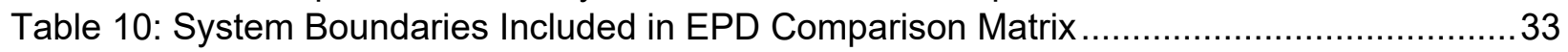

Table 11: EPD Comparison Matrix System Boundaries Second Inputs ...................................33

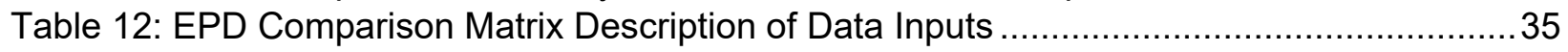

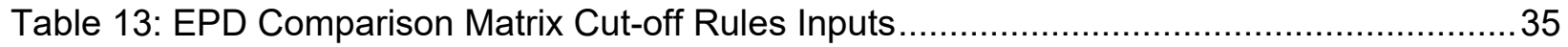

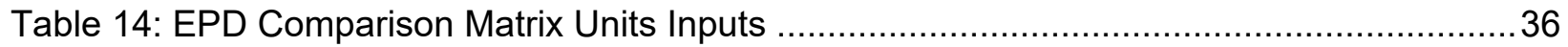

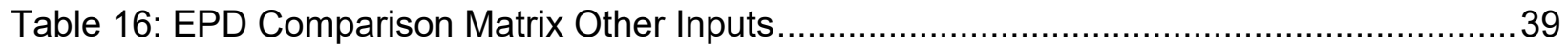

Table 17: EPD Comparison Matrix Comparison Section .................................................... 40

Table 18: System Boundaries Comparison Section Illustrative Example .................................41

Table 19: Summarization Factors in EPD Comparison Matrix.................................................44

Table 20: EPD Summarization Sample Chart.......................................................................46

Table 21: EPD Comparison Matrix Scoring Example ......................................................50

Table 22: EPD Comparison Matrix Scoring Sample ......................................................... 51

Table 23: EPD Comparison Matrix Impact Category Results Sample .................................52

Table 24: PCR Comparison Template based on (Subramanian, Ingwersen \& Hensler, 2011) ..53

Table 25: Sample entries from PCR Comparison Matrix, General Information section ..............56

Table 26: Sample entries from PCR Comparison Matrix, Goal and Scope Definition of LCA

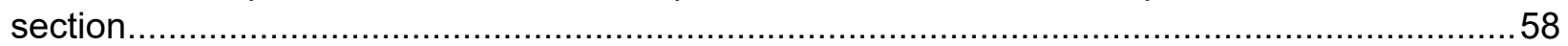

Table 27: Sample entries from PCR Comparison Matrix, System Boundaries section..............60

Table 28: Sample entries from PCR Comparison Matrix, Data Provisions I section..................61

Table 29: Sample entries from PCR Comparison Matrix, Data Provisions II section..................63

Table 30: Sample entries from PCR Comparison Matrix, Life Cycle Impact Assessment section

Table 31: Sample entries from PCR Comparison Matrix, Other Inputs section ..............................66

Table 32: Sample entries from PCR Comparison Matrix Comparison Section.........................67

Table 33: Summarization Factors in EPD Comparison Matrix ............................................71

Table 34: Sample entries from PCR Comparison Matrix, Summarization section....................72

Table 35: Sample entries from PCR Comparison Matrix, Scoring section................................77

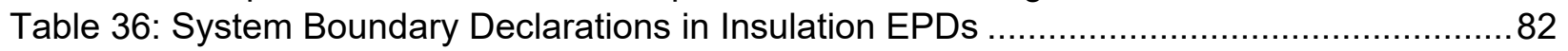

Table 37: Characterization Factors in Insulation EPDs .............................................. 84

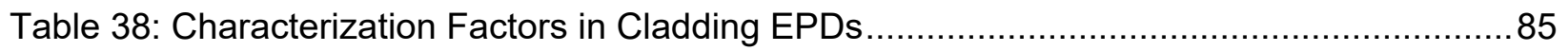

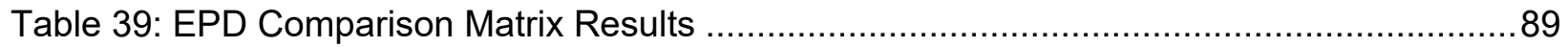

Table 40: EPDs with Same PCR, Invalid Comparison Results .............................................91

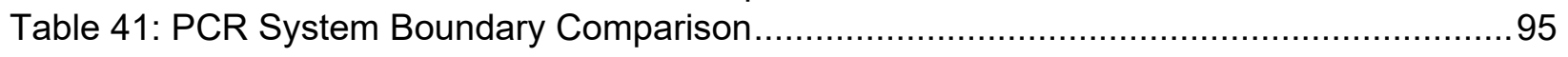

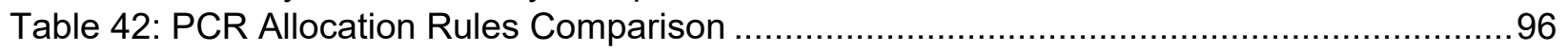


Table 43: PCR Comparison Matrix Results ................................................................ 100

Table 44: PCR Invalid Comparison Results ................................................................ 100

Table 45: Omissions from Simplified EPD Comparison Template ........................................ 104

Table 46: Simplified EPD Comparison Template Excerpt .................................................. 105

Table 47: Reasoning for Invalid Comparisons in EPD Comparison Matrix, Insulation Category

Table 48: Impact Category Characterization Factor Invalidation Comparison, B vs J.............109

Table 49: Impact Category Characterization Factors from PCR, for B vs J Comparison .........110

Table 50: Environmental Aspects that "will" be Declared from PCR, for B vs J Comparison ...110

Table 51: Cut-Off Rules from PCR, B vs J............................................................. 111

Table 52: Best Practice Impact Categories and Characterization Factors ........................... 115

Table 53: Invalidations in Comparisons, EPDs Both Following EN $15804 \ldots \ldots \ldots \ldots \ldots \ldots \ldots \ldots \ldots . . . \ldots 117$

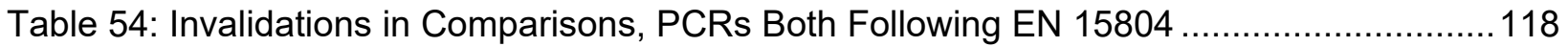




\section{List of Figures}

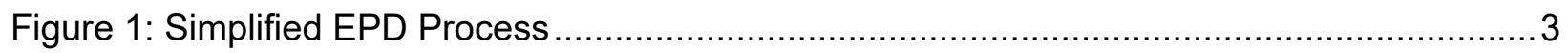

Figure 2: Axonometric drawing of the CaGBC National Office (DIALOG) .............................16

Figure 3: Process Map for Developed Comparison Techniques .............................................25

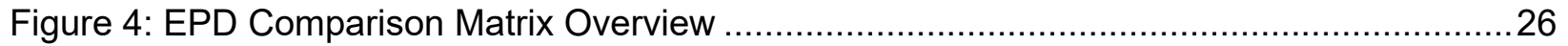

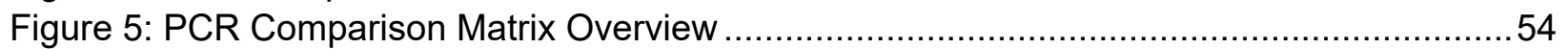

Figure 6: EPD Comparison Matrix Crucial Comparison Results ........................................... 81

Figure 7: EPD Comparison Matrix Non-Critical Content Requiring Caution Results ..................87

Figure 8: PCR Comparison Matrix Crucial Comparison Results...........................................93

Figure 9: PCR Comparison Matrix Non-Critical Content Requiring Caution Comparison Results

Figure 10: Simplified EPD Comparison Template: Tiered Comparison Demonstration 


\section{List of Abbreviations and Glossary}

There are a number of key terms and definitions that must be understood as background for this research. As EPDs are standards-driven documents, they have a unique vernacular, some of which is not present in other sectors of the construction industry, as illustrated in Table 1 below.

Term
Environmental Product
Declaration (EPD)

\section{Definition}

Sometimes called a Type III product declaration, EPDs “[provide] quantified environmental data using predetermined parameters and, where relevant, additional environmental information" (ISO 14025, 2006)

“... compilation and evaluation of the inputs, outputs and the

Life Cycle Analysis (LCA) potential environmental impacts of a product system throughout its lifecycle" (ISO 14040, 2006)

“...phase of life cycle assessment involving the compilation and

Life Cycle Inventory Analysis (LCl)

Life Cycle Impact Assessment (LCIA)

Product Category Rules (PCR)

Program Operator (PO) quantification of inputs and outputs for a product throughout its lifecycle" (ISO 14040, 2006)

“...phase of life cycle assessment aimed at understanding and evaluating the magnitude and significance of the potential environmental impacts for a product system throughout the life cycle of the product" (ISO 14040, 2006)

“...set of specific rules, requirements and guidelines for developing Type III environmental declarations" (ISO 14025, 2006)

"...body or bodies that conduct a Type III environmental declaration programme ... a voluntary programme for the development and use of Type III environmental declarations" (ISO 14025, 2006)

Table 1: Glossary of EPD-specific technical jargon

Other abbreviations used throughout this thesis are listed below.

CaGBC: Canada Green Building Council

EN: Prefix for standard from the European Committee for Standardization

EPD: Environmental Product Declaration

IDP: Integrated Design Process 
ID+C: Interior Design and Construction (LEED® rating system type)

ISO: International Organization for Standardization

LCA: Life Cycle Analysis

LCI: Life Cycle Inventory

LCIA: Life Cycle Impact Assessment

LEED®: Leadership in Energy and Environmental Design

PCR: Product Category Rules

PO: Program Operator 


\section{Introduction}

Industry adoption of Environmental Product Declarations (EPDs, an internationally standardized document providing quantified environmental impacts over the life cycle of a product) is increasing as LEED® $\vee 4$ material credits promote reliance on their content for product comparison and decision-making. This raises the question as to whether this reliance is appropriate, as well as larger questions about how it is affecting the wider construction industry. This research project answers this through two phases of investigation: one, a case study which investigates the use of EPDs on a construction project, and two, a comparability study of construction product EPDs and their underlying PCRs, including insulation, flooring, and cladding products currently on the market.

\subsection{Research Objectives}

The overarching objectives of this thesis are to:

- Develop a better understanding of how environmental product declarations are used in a construction setting to provide best practice guidelines for practitioners;

- Assess currently available environmental product declarations for construction products in a declaration library, and;

- Build a framework for the detailed comparison of environmental product declarations and product category rules based on previous efforts, standards requirements, and ease of use.

\subsection{Research Questions}

To achieve these objectives, this thesis explores the following research questions:

- What are the existing obstacles regarding environmental product declaration adoption and use in the construction industry?

- What are the processes that may enable the comparison of environmental product declarations of two similar products developed using different PCRs?

- To what extent can and should environmental product declarations and product category rules be compared? 


\subsection{Approach}

First, a case study on the first LEED® project in Canada to use EPDs on a construction project and achieve the LEED $®$ Credit Building Product Disclosure and Optimization - Environmental Product Declarations was undertaken. Secondly, small EPD libraries of building construction materials were realized to judge the validity of comparison between EPDs created under different PCRs.

The case study included interviews with three major stakeholders on the project: the Owner/Client, the Designer, and the Contractor. This case study was used to understand how EPDs were used on a construction project: their impacts, their benefits, potential concerns with the methodology and creation of EPDs both now and looking into the future, and the reliance placed on the environmental information within EPDs. The case study is necessarily limited by the fact that the building analyzed is - to the Canada Green Building Council's (CaGBC) knowledge - the first and only LEED $®$ v4 project in Canada to achieve the Building Disclosure and Optimization - Environmental Product Declarations credit. Scorecards from Gold and Platinum LEED® v4 projects from Europe and the United States were examined to identify other potential projects had achieved the credit for additional case studies, but no potential projects were identified.

Based on the findings from this case study, small libraries of EPDs in various product categories specific to construction were created: Insulation, Cladding, and Carpet. These libraries were used to assess the comparability that exists in EPDs and PCRs for construction products using stipulations in standards and guidance documents while awaiting harmonization in North America. A framework for the comparison of EPDs and PCRs was constructed, using these libraries to determine whether sets of EPDs or PCRs are comparable for use by design and construction practitioners. This framework contains matrices for comparing both EPDs and product category rules (PCRs, a set of rules and/or requirements for developing EPDs) for construction products with the same and different PCRs. The level of harmonization of EPDs and PCRs in each product category is analyzed and discussed. Out of this, a comparison template is created in both comprehensive and simplified versions, which will allow practitioners and the industry at large to assess the comparability of two EPDs or PCRs. Products used to populate these libraries were collected from program operator's databases and from general online searches. There was no direct contact with the manufacturers of the products or the publishers of the EPDs and PCRs used in the libraries, and it is not the intention of this research to determine whether the reported information is correct. 


\section{Literature Review}

\subsection{Key Terms and Definitions: The Simplified Claim Process}

The simplified process for creating and publishing an EPD for a product is displayed in Figure 1.

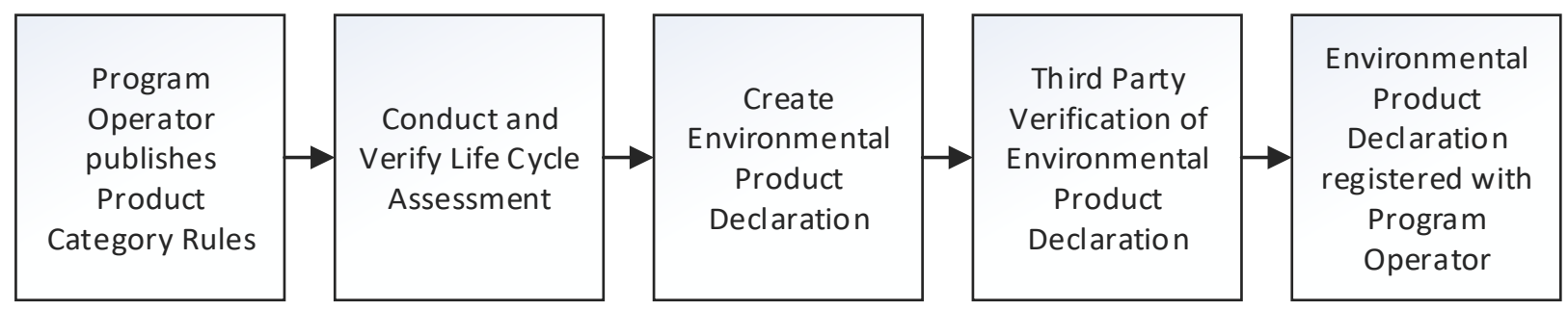

Figure 1: Simplified EPD Process

A Program Operator (PO) is a body overseeing an EPD program by helping manufacturers with the EPD process and registering and publishing EPDs once they are completed. Under the structure of ISO 14025, there is no limit to who can act as a program operator. From the standard: "A programme operator can be a company or a group of companies, industrial sector or trade association, public authorities or agencies, or an independent scientific body or other organization".

Program operators are also responsible for writing and publishing Product Category Rules (PCRs). These PCRs are written for a product category, i.e. a group of products with equivalent functions. PCRs define a specific product category and establish the criteria for elements to be included in an EPD.

In order to obtain the data required in the EPD, a life cycle analysis (LCA) - a methodology to determine environmental impacts of processes and material ingredients throughout a product's life cycle - must be completed and verified by a third party. Once this is done, the EPD is created according to the PCR, verified by a third-party, and subsequently registered with and published by the PO.

\subsection{Materials and the Built Environment}

The emissions stemming from the embodied and operational energy use of the built environment has a substantial effect on the natural environment. In Canada, residential and commercial buildings account for $1 / 3^{\text {rd }}$ of the country's energy use, $50 \%$ of the extracted natural resources, $25 \%$ of landfill waste, $10 \%$ of airborne particulates, and $35 \%$ of greenhouse gas emissions (ISED Canada, 2015), producing 87.2 megatonnes of $\mathrm{CO}_{2}$ eq in 2014 . This is a $20 \%$ 
increase from emissions in 1990 (Environment and Climate Change Canada, 2016). There is a pressing need to reverse the trend of emissions resulting from building construction and operation, and improving the performance of the building stock has been identified as one of the most cost-effective mitigation options of any sector (BPIE, 2011).

Materials used in building construction can help this situation in multiple ways. Environmentallyconscious building products can reduce material use and solid waste through the use of reclaimed, recycled, or reused material, and reduce greenhouse gas emissions through reductions at the manufacturing plant and through construction processes (Ortiz, Castells, Sonnemann, 2008). One of the challenges the construction industry is facing is to be able to identify construction products to assist with this, with trusted and transparent information.

\subsection{Life Cycle Assessment}

Life Cycle Assessment (LCA) is the process by which the global and regional environmental impacts of a product are be quantified. An LCA study can be applied to building materials to decide that is preferable for the project, based on resource use, human health, and ecological consequences.

LCA is used in the manufacturing and design sectors for two separate, but associated reasons by providing quantified data to both: help reduce the environmental impact of products and their associated manufacturing processes, and to help make informed decisions about the embodied energy of buildings, respectively (O'Conner et al., 2012).

There still seems to be a challenge, especially regarding the skills and knowledge gap between the design and engineering industry and life cycle assessment. Glass, et al. (2013) found that while LCA is incredibly useful for robust information on environmental impacts, the use of LCA studies in the engineering industry can be limited due to this knowledge gap. A systematic approach to education, and closer interaction with the manufacturing community can help alleviate this gap. Product documentation is one possible avenue of communication between manufacturer and designer.

There are a number of possible ways LCA data can be communicated in the form of documentation. Jonsson (2000) completed an LCA study as an environmental assessment of flooring products alongside an eco-label, two eco-guides, a product declaration, (Type II environmental claim) and an "environmental concept" (a qualitative evaluation of a product). The study noted that there needed to be balance within the scope and procedure of the document or 
method of study: between high transparency and keeping trade secrets confidential, and between comprehensive results that could be confusing and highly aggregated results that could be misconstrued. Additionally, it was noted that a standardized procedure (such as environmental labelling schemes) may have high credibility but low flexibility, and the use of specific data can provide more useful results. One of the main takeaways from this comparison is that LCA data, and the environmental claims that use LCA data, are the only types of assessments that provide quantitative data; all other assessment schemes or tools can only provide qualitative data.

Even without formal documentation, LCA can also be used to make decisions about materials in the design phase. Means and Guggemos (2015) proposed a framework for LCA-based decision making for buildings in the design phase, but found this type of decision making was more appropriate for materials (like in the case of EPDs for products) rather than whole buildings. Similar findings were found by Haapio and Viitaniemi (2008), where a number of currently available tools for whole building environmental assessment are compared. It was found that most current tools are designed for different types of buildings, rely on different databases, and emphasize different stages of a building's life cycle, limiting the potential for it to be used for whole buildings.

\subsection{Environmental Labelling}

There are three types of environmental labels for products based on LCA data. These all exist within ISO's 14000-series, and include:

1. Type I. Governed by ISO 14024, these are awarded by a third party claiming an environmental preference for a product based on a set of predetermined criteria (ISO 14024:1999). Examples of this are FSC Chain of Custody certification or the EU Ecolabel.

2. Type II. Governed by ISO 14021, these are for self-declared environmental claims. This standard mandates the inclusion of certain information within the claim.

3. Type III, often referred to as EPDs. Governed by ISO 14025, these are third-party verified transparent claims that provide quantified life-cycle information about a product. This thesis focuses on this specific version of environmental claims.

The use of LCA data in environmental labelling schemes has been in use since the early 1990s, and development of the ISO 14000 suite of standards began in 1996 (Ball, 2002). The first 
registered EPD was published for water taps and electrical appliances through the International EPD System in Sweden in 1999. In 2000, the Institute for Environmental Research and Education founded Earthsure in the United States, becoming the first EPD program in North America The use of LCA data in environmental labelling schemes has been in use since the early 1990s (Bergman, Taylor, 2011). Since its inception, the number of EPDs on the market has grown considerably. Currently, there are 3,614 EPDs, 349 of which are for construction products as of 2014 (Hunsager, Bach, Breuer, 2014). The amount of EPD programs has increased across all product categories. For construction products specifically, the amount of EPDs programs has increased from 1 in 2002 to 14 in 2013. Currently, there are 39 different EPD programs, 14 of which (36\%) are for construction products, the sector with the largest share (Minkov, Schneider, Lehmann et al., 2015).

\subsection{ISO 14025 and other Associated Standards and Documents}

As part of this thesis, a detailed investigation of the standards and guidelines surrounding EPDs was completed. The purpose of this section is to provide detailed background on EPD standards and guidelines, when then informs the case study and comparison methodology described in latter sections of this thesis.

\subsubsection{Product Category Rules}

The majority of the existing international standards that govern PCRs also govern EPDs and are described fully in Section 2.5.2.

The Guidance for Product Category Rule Development (2013) is a document that provides instruction to current and potential program operators on how to prepare, publish, and maintain PCRs, self-described as follows:

"This guidance document is a response to an internationally recognized need for additional instruction on the development of rules specific to a category of products for making claims based on a life cycle assessment (LCA). The purpose is to supplement existing standards for LCA-based claims that require the development of product category rules (PCRS) or their equivalents. The aim is that PCRs can be developed in a consistent manner and used to support claims based on multiple standards... The Guidance embodies the efforts of individuals with expertise in LCA and LCA-based product claims from more over 40 organizations in 13 countries and regions under the name of The Product Category Rule Guidance Development Initiative." (PCR GDI, 2013) 
The Guidance for Product Category Rule Development is not an international standard, and does not attempt to pre-empt ISO 14025 or any other standard on environmental claims. Instead, its purpose is to fill gaps in the guidance on PCRs and make the claims process easier, less costly, and less time-intensive. The guidance document does this by supporting adaptation of PCRs and improving comparability of claims through the verbiage and content within the document. The first version of the document was published in 2013 and is described as a living document that will continue to improve as EPD use increases. The document has a strong emphasis on harmonization, a process which unifies current PCRs or makes current PCRs mutually intelligible. This would inherently increase the level of comparability across the PCRs, and strengthen the use of EPDs as true comparative documents.

\subsubsection{Environmental Product Declarations}

Some of the standard documents presented in this section pertain to both PCRs and EPDs, but are included because of the importance within them to EPDs. There are no standards or guidance documents that focus solely on EPDs; there are typically documents that govern PCRs and EPDs in conjunction.

The applicable standards are summarized in Table 2.

There are a number of minor standards that exist that are oft referenced in the above standards, such as "PD CEN/TR 15941:2010 - Sustainability of construction works - Environmental product declarations - Methodology for selection and use of generic data", a supporting document for EN 15804 that provides guidance for the selection and use of generic data within the LCA for an EPD.

A North American standard similar to EN 15804 in intent is currently under development. It is a revision of ISO 21930, with a proposed renaming to "Sustainability in buildings and civil engineering works - Core rules for environmental declaration of construction products and services used in any type of construction works". The draft was published February $11^{\text {th }}, 2016$ and was eligible to be voted on for acceptance until May $10^{\text {th }}, 2016$. At the time of writing, it is still at the close of voting stage as per ISO's directory. 


\begin{tabular}{|c|c|c|}
\hline rd \# & Title & Scope \\
\hline ISO 14025 & $\begin{array}{l}\text { Environmental labels and } \\
\text { declarations - Type III } \\
\text { environmental declarations - } \\
\text { Principles and procedures }\end{array}$ & $\begin{array}{l}\text { An international standard that "establishes the } \\
\text { principles and specifies the procedures for } \\
\text { developing Type III environmental product } \\
\text { declaration programmes and Type III } \\
\text { environmental declarations". This standard } \\
\text { provided most of the guidance for the comparison } \\
\text { chart. }\end{array}$ \\
\hline ISO 14044 & $\begin{array}{l}\text { Environmental management - } \\
\text { Life cycle assessment - } \\
\text { Requirements and guidelines }\end{array}$ & $\begin{array}{l}\text { An oft-cited standard within ISO 14025, which } \\
\text { "specifies requirements and provides guidelines for } \\
\text { life cycle assessment". }\end{array}$ \\
\hline ISO 21930 & $\begin{array}{l}\text { Sustainability in building } \\
\text { construction - Environmental } \\
\text { declaration of building } \\
\text { products }\end{array}$ & $\begin{array}{l}\text { An international standard that "provides the } \\
\text { principles and requirements for Type III } \\
\text { environmental declarations of building products". } \\
\text { This differs from ISO } 14025 \text { because it is building } \\
\text { product specific. }\end{array}$ \\
\hline EN 15804 & $\begin{array}{l}\text { Sustainability of construction } \\
\text { works - Environmental product } \\
\text { declarations - Core rules for } \\
\text { the product category of } \\
\text { construction products }\end{array}$ & $\begin{array}{l}\text { A European standard that "provides core product } \\
\text { category rules (PCR) for Type III environmental } \\
\text { declarations for any construction product and } \\
\text { construction service". This differs from ISO } 14025 \\
\text { and } 21930 \text { because it can act as a PCR for a } \\
\text { product. EN } 15804 \text { was established as a "core } \\
\text { PCR" to establish a higher level of harmonization in } \\
\text { the European building and construction product } \\
\text { market. EN } 15804 \text { is a suite of standards for the } \\
\text { sustainability of construction works, part of which } \\
\text { includes the processes of developing EPDs } \\
\text { (Erlandsson, et al. } 2013 \text { ). Because the } \\
\text { comparability of EPDs is based on having } \\
\text { equivalent PCRs, theoretically, every EPD formed } \\
\text { with EN } 15804 \text { acting as a core PCR will be } \\
\text { comparable. }\end{array}$ \\
\hline
\end{tabular}

Table 2: Titles and Definitions of Standards Related to EPDs and PCRs 


\subsection{Drivers for Environmental Product Declaration Use in Construction}

Increased awareness and concern about environmental impacts, the increasing importance of embodied energy, and the increased awareness of the importance of evaluating product impacts over their life cycle have resulted from increased influence of sustainability and rating systems such as LEED® in the built environment.

With the Building Material Disclosure and Optimization - Environmental Product Declarations credit in LEED $\otimes$ v4, these concerns are addressed by creating incentives for the use of EPDs in green buildings. In this credit, there are two points available. One point ("Option 1") requires the use of 20 or more different products from 5 or more different manufacturers that have EPDs, but dooes not require any specific material performance. A second point is available for the use of products that fall below industry average in three of six impact categories: global warming potential, ozone depletion, acidification, eutrophication, ozone formation, and non-renewable energy depletion. The credit's intent is to transform the market and push manufacturers to create EPDs for products to be used on LEED $®$ projects, and "support(ing) a transition from a single-attribute approach to one that relies on more comprehensive reporting and rewards manufacturers whose products are less harmful to the environment" (LEED $®, 2014)$.

EPDs can also be used as a tool during the design process to allow for comparisons between products of the same product category (Fet, Skaar, Michelsen, 2009. Fet, Skaar, 2006). These comparisons can be completed on the basis that certain criteria within the EPDs are identical and equivalent. This is established within ISO 14025 Section 6.7.2 Requirements for comparability (2006). Examples of EPDs that can be compared are those that are based on LCA covering all life cycle stages, or EPDs based on the same PCR.

\subsection{Environmental Product Declaration Adoption Hindrances}

Given the lack of restrictions on program operators, the number of overlapping PCRs has increased, resulting in inconsistencies between very similar products using dissimilar rules for their EPDs such as differences in LCA methodology or reporting (Ingwersen, Subramanian, 2014). The extent and validity of comparison between such products are limited to the extent that the underlying PCR parameters are comparable. The solution - harmonization - requires the development of EN 15804, mutual recognition of PCRs (Del Borghi, 2012), and/or alignment of PCRs by program operators (Ingwersen, Stevenson, 2012). 
Use of generic rather than specific data can have unfavorable effects on the EPD document (Fet, Skaar, Michelsen 2009), as can poor data quality (Modahl, et al. 2013). ISO 14025 states that some data quality requirements should be "equivalent", but not necessarily identical. Since 'equivalency' is not defined within the standard, generic data could act in place of specific data, even if not recommended (PD CEN/TR 15941, 2010). The use of generic datasets has been found to result in up to $500 \%$ variation in results across environmental impact categories compared with EPDs developed with specific datasets, with this variation directly proportional to the distinctiveness of the process or material (Modahl et al., 2013).

Financial constraints impose another barrier, particularly for smaller manufacturers. Zackrisson (2013) and Fet \& Skaar (2006) identified the lack of EPDs from small and medium-sized enterprises and developed tools for the creation of EPDs for companies and manufacturers who lack the expertise, finances, and personnel to create EPDs for their products. Finally, there is an under-representation of PCRs of North American origin given the size of the construction industry (28\% of PCRs and $20 \%$ of global construction spending) when compared with Europe (55\% of PCRs and $30 \%$ of construction spending) (Minkov et al., 2015. IHS, 2013). The resulting proportional lack of products with EPDs available in North America can make it more challenging for project teams interested in this information at this time, while the volume of products with EPDs in North America is in the process of catching up to the more mature European market.

A lack of harmonized data also poses a hindrance to EPD adoption. As described by Frischknecht, Wyss, Knopfel, and Stolz (2014), life cycle inventory methodology, environmental indicators and life cycle inventory background databases are the key areas for harmonization. However, it was determined that harmonization in these areas is difficult, and may ultimately be unlikely. These conclusions strengthen the need for a tool that could both determine if the comparison of products is appropriate, and facilitate the comparison of products' environmental impacts in lieu of harmonization.

\subsection{Need for Comparison}

This section will explain the methodology for comparing two of the major documents governed by the international standard ISO 14025 - Environmental labels and declarations - Type III environmental declarations - Principles and procedures: EPDs and PCRs.

The definition of comparison in regards to the matrices are: 
- EPD Comparison Matrix: a framework that determine declarations' comparability, and allows for direct comparison, if appropriate.

- PCR Comparison Matrix: a framework that enables the comparison of PCRs to determine large-scale comparability between products and declarations adhering to the specific PCRs.

There are various scenarios in which these matrices would be used. The EPD Comparison Matrix might be of use to an architect, designer, or other practitioner who is interested in comparing the environmental impacts of two or more construction products for use on a project. The PCR Comparison Matrix may be of use to a firm or company that is constantly sourcing a large amount of construction products, and would rather determine the comparability between two or more PCRs to develop a harmonized database, only selecting products from comparable PCRs.

In this thesis, the comparison of EPDs was completed before the comparison of PCRs. This is mainly due to the method of sourcing used: suitable EPDs in each product category were found before PCRs as this was a more efficient method of searching. It is much easier to identify EPDs and find the underlying PCRs used than identify PCRs and find EPDs that have been published using the PCRs.

\subsubsection{Previous Comparison Work}

To determine the direction for the comparison work in this thesis, an analysis of previous comparison work, in regards to both PCRs and EPDs, was completed. The comparison framework was developed as a matrix from a number of standards related to PCRs and EPDs with cues from previous research.

\subsubsection{Comparisons of Product Category Rules}

There have been two known instances of research based on the comparison of PCRs.

The first was the development of the PCR Comparison Template by Subramanian, Ingwersen, and Hensler (2011). This document is "intended for general use in comparison of product category rules (as defined in ISO 14025) or ... for creation of quantitative, life-cycle based environmental labels or declarations of products" (2011). The document is supported by the article titled Comparing product category rules from different programs: learned outcomes towards global alignment (2012), which uses the PCR Comparison Template to compare PCRs from different program operators in multiple sectors, including milk/dairy, horticultural products, 
wood-particleboard, and laundry detergent. The PCR Comparison Template is a Word document that has five main columns: a criteria column (the component being compared in the row), a description column (a short description of the information required within the row), two columns for the required information from both PCRs being compared, and a comments column for any general notes not to be included in the other columns. An excerpt from this chart is shown below.

\begin{tabular}{|c|c|c|c|c|}
\hline CRIIER/A & DESCRPION & PCR\#1 & PCR\#2 & COMMENTS \\
\hline \multicolumn{5}{|l|}{$\begin{array}{l}\text { General } \\
\text { Information }\end{array}$} \\
\hline Product & $\begin{array}{l}\text { Provide the name of the } \\
\text { product category that } \\
\text { this } P C R \text { is targeted at }\end{array}$ & & & \\
\hline $\begin{array}{l}\text { CPC Module (if } \\
\text { applicable) }\end{array}$ & $\begin{array}{l}\text { Provide the entire } \\
\text { hierarchy (names and } \\
\text { codes) that leads up to } \\
\text { the product category } \\
\text { and its categories if } \\
\text { applic able }\end{array}$ & & & \\
\hline $\begin{array}{l}\text { UNSPSC } \\
\text { Module (if } \\
\text { applicable) }\end{array}$ & $\begin{array}{l}\text { Provide the entire } \\
\text { hierarchy (names and } \\
\text { codes) that leads up to } \\
\text { the product category } \\
\text { and itscategories if } \\
\text { applic able }\end{array}$ & & & \\
\hline $\begin{array}{l}\text { GPC Module (if } \\
\text { applicable) }\end{array}$ & $\begin{array}{l}\text { Provide the entire } \\
\text { hierarchy (names and } \\
\text { codes) that leads up to } \\
\text { the product category } \\
\text { and itscategores if } \\
\text { applic able }\end{array}$ & & & \\
\hline
\end{tabular}

Table 3: PCR Comparison Template (Subramanian, Ingwersen \& Hensler, 2011)

The chart contains six major categories of information required: General Information (some of which is shown in the table above), Goal and Scope (the majority of the components within the LCA: system boundaries, data quality, etc), Life Cycle Inventory Analysis (inventory parameters and analysis), Life Cycle Impact Assessment (impact indicators and characterization factors, normalization and weighting rules), Communication (format and structure of the EPD), and Miscellaneous (additional environmental information, safety information, glossary, referenced literature).

The major conclusion found by this study was that the deficiencies in comparison ranged from general differences in scope, system boundaries, environmental impacts. Even with all the variances identified, the authors saw that there was no clear rationale as to why the PCRs analyzed (and the PCR sector in general) could not be consistent in the future if alignment 
procedures were followed. It was concluded that most of these problems could be attributed to the independent development of PCRs, differences in purpose, and variance in overarching standards.

The second attempt at PCR Comparison was completed by Minkov et al. (2015) in the article titled Type III Environmental Programmes and harmonization of product category rules: status quo and practical challenges. This is less of a review of existing program operators, their EPD programs, and their conformance to ISO 14025 rather than a comparison of PCRs. It found that $75 \%$ of PCRs are fully ISO-conformant, with the deficiencies due to differences in terminology and non-compliance to ISO 14025 based on criteria established in the Conformity Assessment Form, which supplements the Guidance for Product Category Rule Development by extracting the requirements and recommendations for PCRs from the guidance document.

\subsubsection{Comparisons of Environmental Product Declarations}

Hunsager, Bach, and Breuer (2014) completed a study to identify harmonization potential by mapping existing PCR and EPD documents. The authors identified 27 programs, 556 different PCRs, and 3,614 EPD declarations, and analyzed each in regards to their region, validity, and sector, among others. The study found that construction products and services was the fourth largest sector for EPDs, and the program operators in the sector were relatively efficient. However, the authors acknowledged there was a problem in the categorization of products in the construction industry, as construction products do not easily fit into the UN CPC (United Nations Central Product Classification, an international classification system for goods and services) material based system. The nature of the study only allowed for a focus on these types of macro characteristics but included an interesting discussion regarding improvements to EPD verification and harmonization. Regarding verification, the largest improvement to be gained is through a greater level of transparency in EPD verification to assure consumers that the published data are correct. This could be accomplished by guaranteeing the involvement of certain stakeholders in the process, and ensuring that third-party verification was completed by a separate and independent third-party, with requirements for reviewers to declare possible conflicts of interest. With harmonization, the most important component is procedural alignment, an example of which is the creation of a "core PCR" (EN 15804) in Europe for construction products. However, this core PCR is carried out in differing program structures (program operators will use the PCR but adapt it for their own market/geographic needs), and may be in conflict with non-European programs and products that do not follow the core PCR. For these bodies, it is recommended that the mandatory involvement of consumer and environmental 
interests in the review panel be considered, and implementing a mandatory external third-party verification process for all declarations for use by the general public.

\subsubsection{Gap Analysis}

The basis of comparability within EPDs is found in ISO 14025 Section 5.6: "Type III environmental declarations not based on an LCA covering all life cycle stages, or based on different PCR, are examples of declarations that have limited comparability". This clause, however, precludes the majority of comparison that would happen between EPDs.

Currently, there is no known research on two key topics of interest to the study: first, research regarding the comparability between EPDs based on the content within rather than whether they are based on the same PCR, and secondly, user-friendly comparison for designers, rather than LCA practitioners. Additionally, most of the EPD comparison work has been done on a macroscale, comparing a large number of EPDs to focus on market trends. There is minimal research based on micro-scale comparison, an attempt to compare EPDs while awaiting an appropriate level of harmonization in North America.

The micro-scale comparison that exists a) only exists for the comparison of PCRs and b) is manual and dependent on the users' skill, knowledge, and familiarity levels. There is a gap in user-friendly, semi-automated comparison techniques with the potential for inputs for comparing multiple products that would be of great use to design practitioners. This is the basis on which the Comparison Matrices in this thesis have been developed, using factorial comparison to compare each EPD or PCR within the matrix to every other (i.e. $A$ vs $B, A$ vs $C, A$ vs $D, B$ vs $C$, $B$ vs $D, C$ vs D) with automation based on the user's input. 


\section{Case Study: Canada Green Building Council National}

\section{Office, Vancouver, BC}

In order to identify ways to improve how EPDs are used in the construction industry, it is important to understand how they have been previously implemented. To do this, a case study of a Canadian construction project has been undertaken to investigate how these declarations are used through the design and construction process. This case study was used to frame and inform the succeeding steps within this thesis, which aim to ease the process of material selection for designers and identify key areas for practitioners to be critical for comparison within EPDs.

Only one case study regarding the use of EPDs has been completed. This is because at the time of writing this project was the first, and to the best of CaGBC's knowledge, the only LEED尺 v4 project in Canada to achieve the Building Disclosure and Optimization credit for EPDs. There were attempts to identify potential projects from the United States and Europe that have achieved the credit but none could be found.

\subsection{Background and Method}

The CaGBC office relocation to the newly constructed MNP Tower in downtown Vancouver is slated to be the first LEED® v4 Interior Design and Construction (ID+C) Gold project in Canada, with Platinum as a stretch target. At the time of writing, the certification process is still ongoing. The project obtained occupancy on September 8th, 2015. Located on the south side of the tower on the fifth floor, the project has a total area of $3,100 \mathrm{ft}^{2}\left(288 \mathrm{~m}^{2}\right)$. As the office for the CaGBC, this space is intended to serve as a showcase for other LEED® projects in Canada, CaGBC members, visitors, and others who are interested in sustainability in the built environment. The building achieved this by considering transparency in the office, and flexibility in static and dynamic exhibit areas. Key goals of this ID+C project involved strengthening the link with the CaGBC Ottawa office, providing a space for meeting and collaboration that CaGBC staff could be proud of, showcasing LEED® strategies in a professional setting, providing a healthy space that promotes the well-being of the staff, and showing regional nature through materiality. 


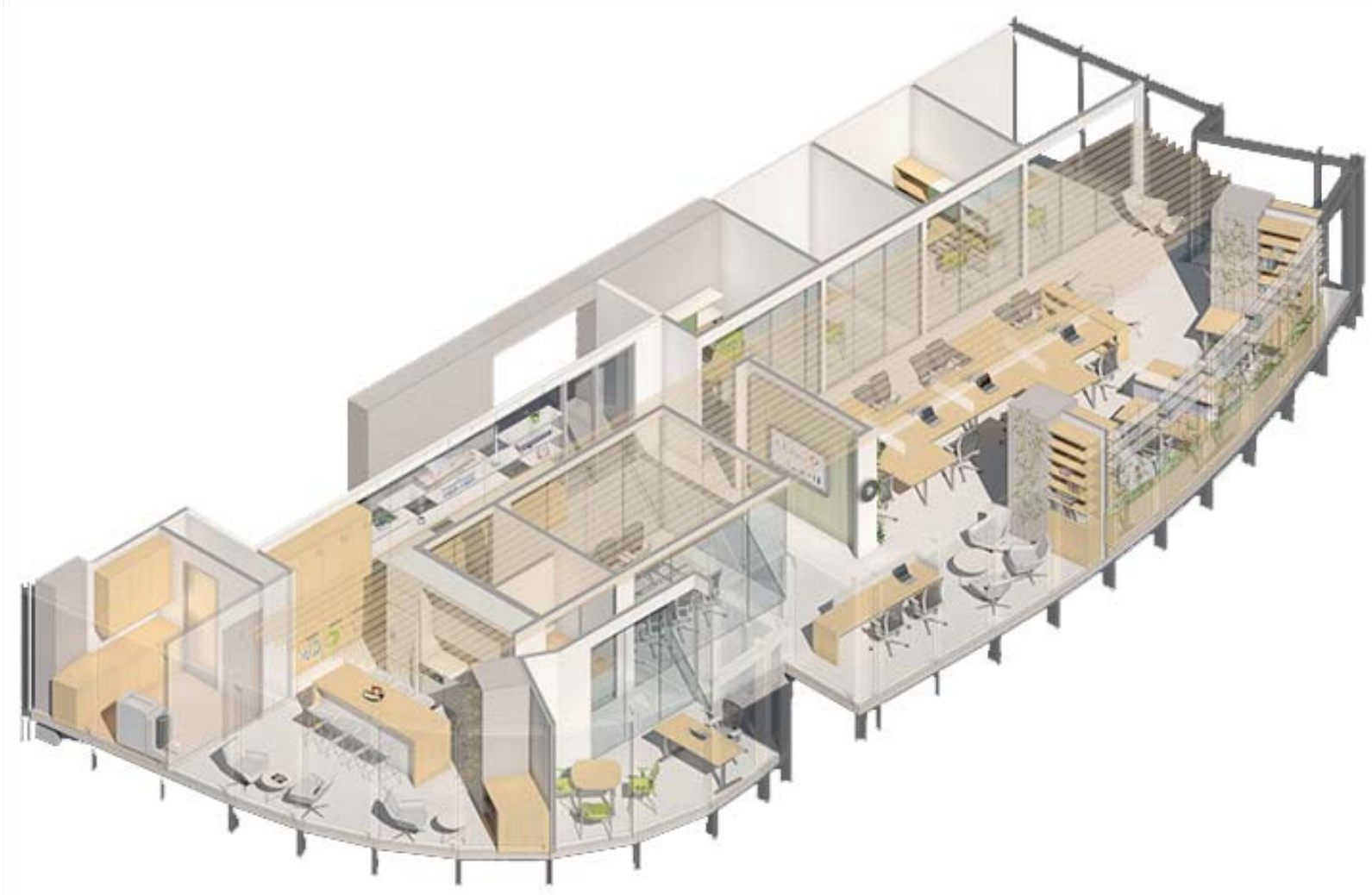

Figure 2: Axonometric drawing of the CaGBC National Office (DIALOG)

This renovation was selected for this case study as it is set to be the first Canadian project to achieve the Building Disclosure and Optimization credit focused on EPDs. This credit category has two options for credits, presented in the Table 4 below (LEED®, 2014).

Three key project participants - the Owner/Client (CaGBC), the Designer (DIALOG), and the Contractor (Ledcor) - agreed to participate in semi-structured interviews. One employee from CaGBC, two employees from DIALOG, and two employees from Ledcor, each of whom were significantly involved with the project were recruited to complete the interviews. For confidentiality reasons, their names and specific job titles have been omitted from this paper.

These interviews focused on key topics related to the use of EPDs in a construction project, including experience with EPDs and material sourcing before the CaGBC National Office project, driving forces behind pursuing the LEED $®$ credit, the impacts of EPDs on the project, benefits of using EPDs on the project, drawbacks of using EPDs on the project, concerns with certain aspects of EPDs, and willingness to work with EPDs in the future. 


\begin{tabular}{|c|c|}
\hline $\begin{array}{l}\text { Option 1: Environmental Product } \\
\text { Declaration (1 point) }\end{array}$ & Option 2: Multi-Attribute Optimization (1 point) \\
\hline $\begin{array}{l}\text { Use at least } 20 \text { different permanently } \\
\text { installed products sourced from at least } \\
\text { five different manufacturers that meet } \\
\text { one of the disclosure criteria below. }\end{array}$ & $\begin{array}{l}\text { Use products that comply with one of the } \\
\text { criteria below for } 50 \% \text {, by cost, of the total } \\
\text { value of permanently installed products in the } \\
\text { project. Products will be valued as below. }\end{array}$ \\
\hline $\begin{array}{l}\text { Product-specific declaration } \\
\text { Products with a publicly available, } \\
\text { critically reviewed life-cycle } \\
\text { assessment conforming to ISO } 14044 \\
\text { that have at least a cradle to gate } \\
\text { scope are valued as one quarter }(1 / 4) \\
\text { of a product for the purposes of credit } \\
\text { achievement calculation. }\end{array}$ & \\
\hline $\begin{array}{l}\text { Environmental Product Declarations which } \\
\text { conform to ISO 14025, 14040, 14044, and } \\
\text { EN } 15804 \text { or ISO } 21930 \\
\text { and have at least a cradle to gate scope. } \\
\text { - Industry-wide (generic) EPD - } \\
\text { Products with third-party certification } \\
\text { (Type III), including external } \\
\text { verification, in which the manufacturer } \\
\text { is explicitly recognized as a participant } \\
\text { by the program operator are valued as } \\
\text { one half (1/2) of a product for } \\
\text { purposes of credit achievement } \\
\text { calculation. } \\
\text { Product-specific Type III EPD - Products with } \\
\text { third-party certification (Type III), including } \\
\text { external verification in which the manufacturer } \\
\text { is explicitly recognized as the participant by } \\
\text { the program operator are valued as one } \\
\text { whole product for purposes of credit } \\
\text { achievement calculation. }\end{array}$ & $\begin{array}{l}\text { Third party certified products that } \\
\text { demonstrate impact reduction below industry } \\
\text { average in at least three of the following } \\
\text { categories are valued at } 100 \% \text { of their cost for } \\
\text { credit achievement calculations. } \\
\text { - global warming potential (greenhouse } \\
\text { gases), in } \mathrm{CO}_{2} \mathrm{e} \text {; } \\
\text { - depletion of the stratospheric ozone layer, } \\
\text { in kg CFC-11; } \\
\text { - } \quad \text { acidification of land and water sources, in } \\
\text { moles } \mathrm{H}+\text { or kg } \mathrm{SO}_{2} \text {; } \\
\text { - eutrophication, in kg nitrogen or kg } \\
\text { phosphate; } \\
\text { - formation of tropospheric ozone, in kg } \\
\mathrm{NO}_{\mathrm{x}}, \mathrm{kg} \mathrm{O}_{3}, \text { or kg ethene; } \\
\text { depletion of nonrenewable energy } \\
\text { resources, in } \mathrm{MJ} \text {. }\end{array}$ \\
\hline $\begin{array}{l}\text { USGBC approved program } \\
\text { Products that comply with other USGBC } \\
\text { approved environmental product declaration } \\
\text { frameworks. }\end{array}$ & $\begin{array}{l}\text { USGBC approved program } \\
\text { Products that comply with other USGBC approved } \\
\text { multi-attribute frameworks. }\end{array}$ \\
\hline
\end{tabular}

Table 4: Building Disclosure and Optimization - Environmental Product Declarations Credit Details 


\subsection{Case Study Findings}

\subsubsection{Experience with Environmental Product Declarations}

All interviewees noted they were familiar with EPDs, but had no experience working with them on a previous project. DIALOG indicated material sourcing was a day-to-day task, but EPDs did not enter the conversation of material sourcing and selection until the CaGBC National Office project.

\subsubsection{Driving Forces Behind LEED® Credit}

The CaGBC wanted the designer to understand and minimize the environmental impact of their material selections and identified EPDs as the preferred tool to do so. In an ID+C project such as this one, there is minimal ability to decrease the environmental impact of the structure or envelope, so the tenant must focus on reducing environmental impact in the interior finishes and furnishings used on the project.

The Owner had three additional objectives for pursuing this credit, aimed at demonstrating the implementation of EPDs on an exemplary project to the green building community. First, they wished to reward manufacturers who had been pioneers in creating EPDs for building products, noting that such manufacturers would have been thinking about lessening their environmental impact and tracking the resource extraction and manufacturing process data to generate the LCA for the EPDs for a substantial period of time. Second, they wanted to demonstrate the feasibility of pursuing and achieving the credit to the green building industry. Third, CaGBC wanted to understand what the challenges of meeting this credit were from first-hand experience and better understand how the credit affected the overall process including design and material sourcing.

Apart from CaGBC's request for sustainable materials, DIALOG saw the EPD credit as necessary to achieve the LEED® Platinum "stretch goal" for the project. Using EPDs was part of the team's strategy to maximize the number of credits wherever possible, paying close attention to the Materials and Resources credits new to LEED® v4 and yet to be widely adopted.

\subsubsection{Impacts of Using Environmental Product Declarations on a Project}

For DIALOG, one impact of using EPDs was conducting much more material research than they would have on a comparable project that did not rely on EPDs. Not only did this change the material choices for the project, but it changed the conversation for all of DIALOG's current and 
future projects. Using EPDs forces designers to look at LCA and strategies within the EPD much more in-depth than previously possible with other labeling schemes.

DIALOG noted a necessary increase in interaction with manufacturers due to the documentation requirements for the EPD credit. The team found that manufacturers interested in having their product used on the project were helpful, providing all the required documentation promptly. DIALOG stated that products that have EPDs come from manufacturers who have an environmental story they want to tell, which the team found helpful. However, they believed that as EPDs increase in both use and availability, these early adopter characteristics are unlikely to hold and thus practitioners will need to be more cautious regarding who they are working with. In addition to the manufacturer interaction, DIALOG found that EPD inclusion required specifications to be written differently than in other projects. Usually, the team would write an open-ended specification where contractors could choose any manufacturer alternate from a generic term, as long as the product met or exceeded performance criteria. However, when EPDs were used, the specification had to be written very tightly. Instead of using the standard specification language seen in most projects, the product(s) named in the specification had to be the product(s) the contractors used. Further, Ledcor was required to source compliant products where none were listed.

EPDs affected discussions related to materials within the project team. DIALOG did not verify whether the PCRs of the declarations and the LCA of the products lined up but used the information and data from EPDs towards analysis at a whole-building perspective: EPDs were referred to as one source of information about a product's overall performance taken into consideration along with other sources of product information, rather than being used to directly compare two similar products. Many EPDs state that comparability of their product to others is reliant upon EPDs having the same PCRs, because different PCRs will have varying requirements for the LCA data reported in the EPDs. DIALOG did mention that if the team was attempting to meet the second point in the credit category regarding environmental performance, the data in the EPD would have had influence on material selection more.

DIALOG stressed the importance of the Integrative Design Process, a holistic approach to the design and construction of a building, by learning about EPDs and the nuances of the credit at the beginning of the project timeline. This was helpful not only for the overall design goals, but for the goals specific to material sourcing and use. The team reiterated that if a project had LEED $®$ Platinum as a goal or target, the project team would "need to learn (about EPDs) first, as it has a trickle-down effect to the rest of (the) project." In addition to this, the team found 
products needed to achieve two or more objectives. It was not enough for the product to only have an EPD to be useful to the project. The best products would have documentation that would help achieve all of the Building Product Disclosure and Optimization credits, including manufacturer inventories, health product declarations, Cradle to Cradle certifications, or corporate sustainability reports.

The overall project team found that overall, the EPD credit was a difficult credit to achieve. As an ID+C project, 20 materials is a relatively large number of products. DIALOG noted on wholebuilding projects (such as $B D+C$ ) where the structure and envelope could be included, the EPD credit would be easier to achieve. As a precautionary measure, DIALOG included a buffer of approximately 5 products in the material selections in case one of the materials that was submitted for approval did not qualify for the credit. DIALOG had to make use of this buffer during the construction project and found this worked to their advantage to achieve the credit.

\subsection{Results}

\subsubsection{Benefits of Using Environmental Product Declarations on a Project}

Key benefits from DIALOG's perspective were that: (1) the fact that EPDs were verified documents about environmental impacts, (2) EPDs helped the team make informed decisions, adding depth to selection discussions by providing the necessary information, and (3) the use of EPDs also raised the awareness and education level in the office, as it increased the availability of transparent material information and data. DIALOG thus concluded that EPDs gave them the opportunity to speak to the sustainability of the project much more comprehensively, providing quantifiable data to reinforce their claims where no policy or advocacy already existed to do so.

The key benefits to the contractor were (1) improved transparency on material performance claims, and (2) consistency through the use of a standard protocol (ISO 14025:2006). In addition, one interviewee noted that those materials with EPDs met or exceeded the expectations for overall quality and sustainability.

\subsubsection{Drawbacks of Using Environmental Product Declarations on a Project}

Both DIALOG and Ledcor noted drawbacks with the use of EPDs: Ledcor focused on logisticstype responses and DIALOG on sourcing and design issues.

While many of the products were donated to $\mathrm{CaGBC}$ and thus it would be hard to determine a total cost increase, Ledcor noticed a definite upcharge with some of the products with EPDs on 
their end and when speaking with their subcontractors. In addition to this, there were some products that had to come from longer distances than on other projects, which lead to increased shipping costs and longer lead-times incurred on the project. Products were also difficult to source, and this process took longer at the beginning of the project. The subtrades first submitted documentation that was LEED® 2009 - but not v4 - compliant. As the project went on, the team was better prepared to find compliant products to get the EPD credit.

Some products, such as carpet or tile, have their warranties dependent on the use of certain adhesives or sealants that created problems for Ledcor. These adhesives and sealants did not carry EPDs with them. Further, some did not meet testing requirements for other LEED® credits, such as the Low Emitting Materials credit. This makes the entire process more difficult, since aligning multiple products with these various requirements becomes a time-intensive task. An employee from Ledcor noted that ideally a product with an EPD should be LEEDRcompliant, and have EPDs for all the required components of that same product. This would also help project teams, since a carpet and an adhesive would count as two separate products towards the credit total. In closing, Ledcor said that best practice on future projects would be to jump on all of the material ordering first to make sure all products arrived on time.

During the design process, DIALOG started to look at products from Europe, but found balancing the different rating and reporting systems to be cost-prohibitive and challenging. Other products were considered based on the assumption of availability in North America, only to learn they were manufactured or only available in Europe. Additionally, some manufacturers positioned themselves to focus on the European market rather than the North American market. The products from North America were preferable in terms of availability, cost (shipping and lead-times), and environmental impact (related to transport distances).

DIALOG also indicated there were fewer EPDs available from small- to medium-sized manufacturers. Larger manufacturers sometimes have someone on staff who is knowledgeable and can take on the process for creating EPDs, or at least have the financial capabilities to do so externally. Small- to medium-sized manufacturers are restricted in this regard (Fet, Skaar, Michelsen, 2009. PE CEN/TR, 2010) and this has an effect on the total availability of EPDs in the building product market.

\subsubsection{Concerns with Aspects of Environmental Product Declarations}

The effect PCRs and LCA have on the use of EPDs has been extensively studied in the literature, concerning PCR variability (Minkov et al., 2015. Subramanian, Ingwersen, Hensler, 
2012) and LCA methodology (Del Borghi, 2012. Modahl et al., 2013). DIALOG staff held a neutral opinion on the variability of PCRs, as they believe they need to see more products with differing PCRs to get a stronger sense of the variability. Regarding LCA methodology, they believed there needed to be some flexibility in order for products to get EPDs. However, they mentioned there was some concern with trusting manufacturers to make EPDs with consistent, comparable, or true LCA data, noting that as soon as more manufacturers start the process and learn the rules of creating EPDs, they will find shortcuts or workarounds that may affect their quality.

DIALOG also had concerns about transparency and how EPDs are analyzed. While acknowledging the positive impact of EPDs on the building industry and as a good source of information, they stressed the importance of reading them critically. One example the interviewees gave was a hypothetical product that had an independently verified Type III EPD and scored exceptionally well in the environmental impact criteria, but may have a process or material that is socially unethical in the manufacturing chain. Some EPDs disclose locations of material sources, or other relevant information regarding manufacturing, while others do not. Making decisions about socially ethical products would be hard to ascertain for someone who is not extremely knowledgeable on the topic.

One particular area under scrutiny by DIALOG is that Option 1 - worth one LEED® credit - is available for the use of 20+ different products from 5 different manufacturers, regardless of the environmental excellence (or lack thereof) of the product. This option is focused on market transformation and is intended simply to incentivize manufacturers to create EPDs for building products. As a result, a product could have very poor environmental performance, but still qualify for the LEED $®$ credit, provided that it has an EPD. This was of concern to the team, however they recognized the implementation of transparency into LEED $®$ as a good introduction into the rating system, and predict rewarding environmental performance within EPDs as the next step. While this is currently part of the LEED® EPD credit, the team sees this emerging as the key qualifier for the credit in future versions of LEED $\circledast$.

Ledcor's main concerns dealt with the shipping distances, costs, and lengthy lead-time products with EPDs had. One interviewee noted that it was hard to see the real benefit of EPDs, as materials were shipped long distances (thus increasing embodied energy due to transportation) simply because they carried an EPD. 


\subsubsection{Willingness to Work with Environmental Product Declarations in the Future}

Both the DIALOG and Ledcor indicated they would work with EPDs in the future on LEED $\circledast$ projects. The Contactor believed it is a credit they should be targeting, especially in regards to the quality of the materials with EPDs. However, Ledcor noted there would need to be a process change, requiring an early thorough specification review to identify specific products with longer lead-times and potential incompatibility between associated products (like adhesives and sealants) and LEED $®$ requirements.

DIALOG felt they spent a lot of time learning and becoming familiar with the concept and were in favor of a big push in the use of EPDs in future work. They added to this by saying in the next whole-building project, products with EPDs will help inform whole-building LCA and make material selection easier. DIALOG also indicated a key impact of the case study project was to trigger an overhaul of their material specification process to incorporate EPDs, including how they both specify materials and categorize specifications.

However, both companies felt there would be hesitation with using EPDs on non-LEED® projects. Ledcor mentioned the scheduling and budget impacts associated with their use, while DIALOG noted a lot of clients would want the background knowledge that comes with EPDs, but would not necessarily want to implement it in a non-LEED $®$ project. Even though this is true, the DIALOG stated that as they work on more LEED® projects, the non-LEED® projects benefit from this over time, since knowing which products are environmentally friendly because of their EPDs and other product documentation only makes professionals better.

\subsection{Case Study Conclusions and Recommendations}

The following preliminary conclusions were drawn from this case study:

1) EPDs are helpful for designers as the independently-verified data is useful to justify specific material selections based on environmental performance; similarly, this transparency is of value to contractors.

2) By using EPDs to make whole building decisions rather directly compare materials and thus inform selections, issues of comparability and level of harmonization between EPDs may be avoided. 
3) The number of EPDs in the North American market is limited, further restricting both comparison and material choice, adding a level of complexity to interior renovation projects seeking to use a large number of EPD-labelled products.

4) The lack of EPD harmonization between some products and warranty requirements (e.g. specified sealants) can result in an otherwise well-performing product being excluded from consideration.

5) The LEED\& $\vee 4$ credit provides incentives both for manufacturers to create EPDs and practitioners to implement it in construction projects.

The authors acknowledge that a single case study does not allow conclusions to be drawn to the industry as a whole, however, the completion of additional case studies as EPD use increases will validate or correct the above conclusions. Future research could potentially revisit this project once LEED® certification has been achieved to identify any additional issues arising and consolidate lessons learned.

There is a strong need for harmonization in PCRs that would lead to increased comparability in EPDs, an advancement that would strengthen the role of EPDs as a design tool. In this stage before harmonization, practitioners should be cautioned with total reliance on EPDs for direct comparison, unless the underlying LCA and PCRs have been thoroughly reviewed. This has led to the development of a formal methodology to compare EPDs without harmonization, including a template for the comparison of underlying PCRs, which is presented in the following sections of this thesis. 


\section{Design of the Environmental Product Declaration}

\section{Comparison Matrix}

\subsection{Matrix Development Methodology}

The development of the EPD Comparison Matrix has been informed by the previous literature (see Section 2.8.1.1 and 2.8.1.2). The process for the development of the EPD Comparison Matrix, PCR Comparison Matrix, and the resulting Simplified Templates within this thesis is illustrated in Figure 3.

INFORMATIVE RESEARCH

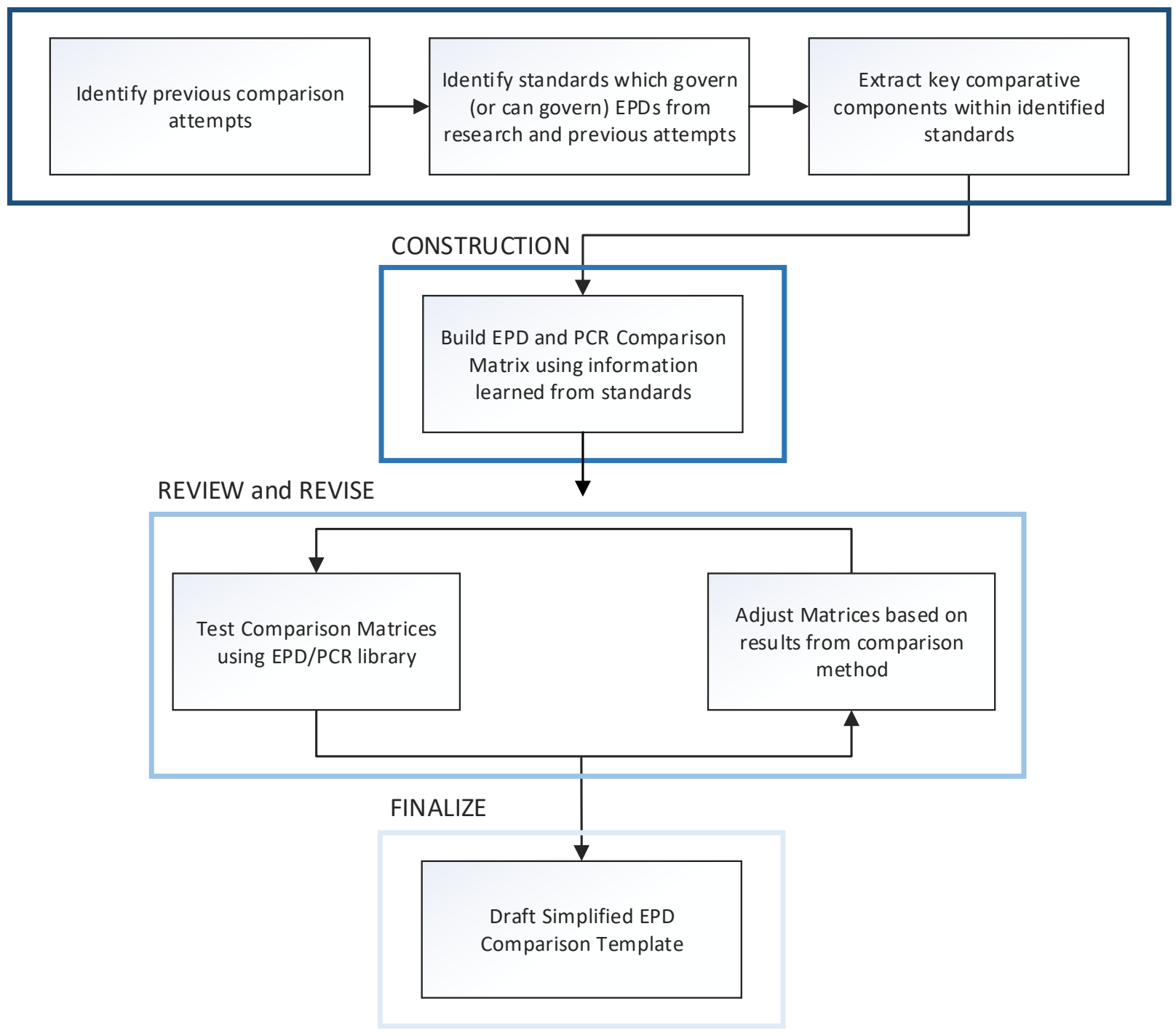

Figure 3: Process Map for Developed Comparison Techniques 
The matrix includes cues from the following standards (refer to Section 2.5.2): ISO 14025, ISO 14044, ISO 21930, and EN 15804. The EPDs analyzed in this chart did not necessarily follow each of these standards. However, in this context, the cues from each standard are included as best practice guides for good quality EPDs for construction products. Each EPD must follow ISO 14025 (theoretically, there are instances where documents are mislabelled as EPDs that do not follow ISO 14025). However, there are some components of a good quality EPD that are not explicitly stipulated in ISO 14025, so other similar standards were also referenced to build the matrix. An example of this is the System Boundaries section, which was adapted from EN 15804 Section 6.2. ISO 14025 does not stipulate the possible system boundaries that can be used in the EPD, as the standard is written for all possible products/services that can achieve EPDs. EN 15804 is a standard specific to construction and has system boundaries for construction products within the standard (e.g. installation, de-construction).

Figure 4 below illustrates an overview of the EPD Comparison Matrix. Each of the chart's separate sections and functions are explained in this section. The following sections discuss each component.

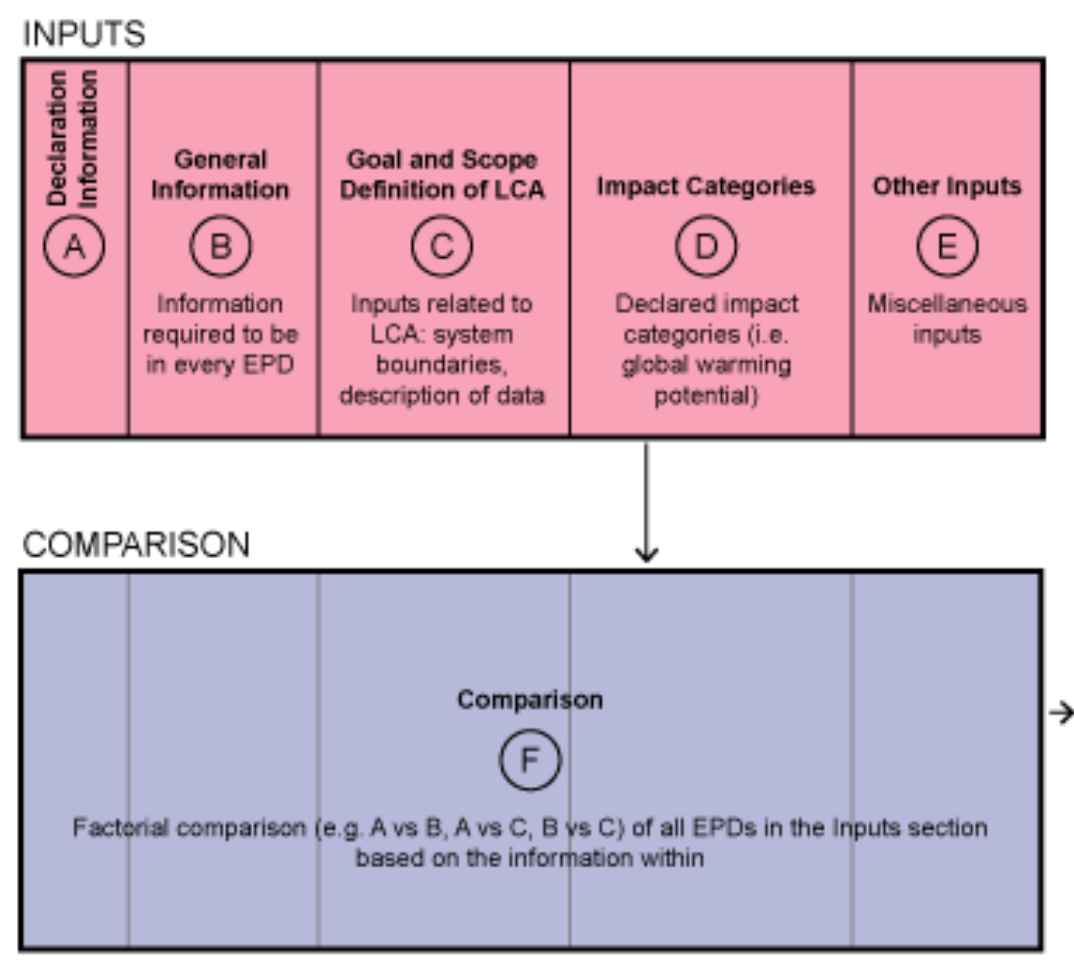

IMPACT CATEGORY RESULTS

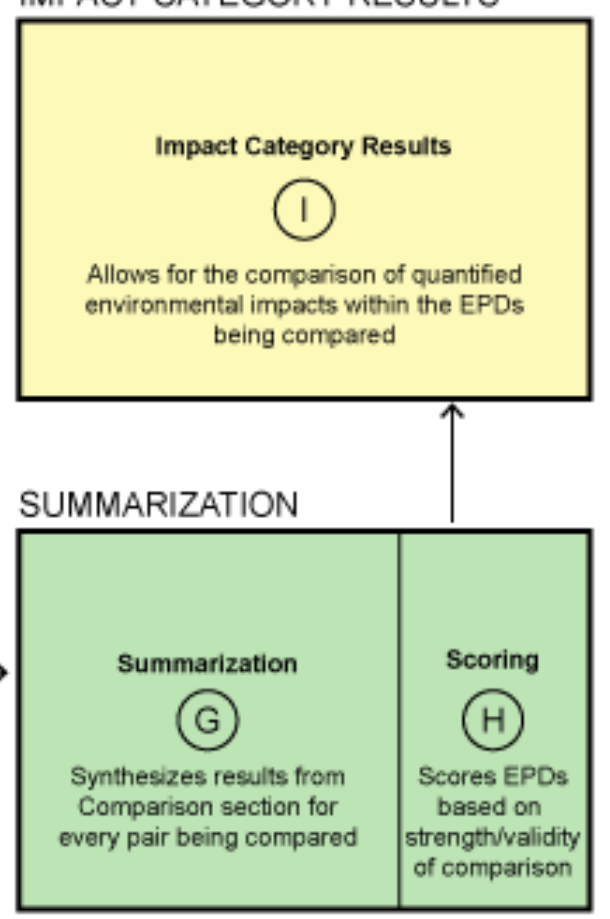

Figure 4: EPD Comparison Matrix Overview 
Parts A-E (described in Section 4.1) discuss the inputs. Part A contains Declaration Information, which includes basic information on the declaration (such as the product and manufacturer name) and the governing standards; this is discussed in Section 4.1.1. Part B covers General Information, i.e. required information in each EPD as defined by ISO 14025 Section 7.2; this is discussed in Section 4.1.2. Part C contains elements from the Goal and Scope Definition for LCA as defined by ISO 14025 Section 6.7.2 and is elaborated on in Section 4.1.3. Part D focuses on Impact Categories, based on information within the standard EN 15804 Section 6.4.3; this is discussed in Section 4.1.4. Part E is comprised of Other Inputs for comparison that do not fit into any of the other categories, and is discussed in Section 4.1.5.

Part $\mathrm{F}$ describes the Comparison process that uses the information within the Inputs section to make detailed comparisons for every critical component of the EPD Comparison Matrix, and is discussed in Section 4.2. Each section that is included in Section 4.1 is reflected in Section 4.2 as subsections, where the comparison process is described in detail.

Parts $\mathrm{G}$ and $\mathrm{H}$ (described in Section 4.3) focus on the Summarization stage of the matrix, which uses the detailed Comparison section to provide overviews for items within the matrix whose inclusions are crucial, or should be compared with caution. Part $G$ focuses on the summarization aspect of this section and is described in Section 4.3.1, while Part $\mathrm{H}$ discusses the scoring process for each comparison in the matrix and is elaborated on in Section 4.3.2.

Part I presents the Impact Category Results section, and is discussed in Section 4.4. Essentially, this table compiles the impact categories placed in the matrix in the Inputs stage, and allows the user to input the quantified impacts from the EPD to use for comparative purposes.

\subsection{Inputs}

To begin the process of comparing EPDs, the content necessary for comparison needed to be identified. Cues were taken from the standards outlined in Section 3.1, which include ISO 14025, ISO 14044, ISO 21930, and EN 15804. The content, language, and terms used in the matrix were taken from the relevant international standards, with the majority of the content for the matrix taken from ISO 14025; the other remaining standards and guidance documents added other metrics for comparison where appropriate for specifically for construction products. Additionally, some comparison metrics that were not included in any of these standards, but were judged as important characteristics for the comparability of EPDs were included in the matrix. These comparison metrics did not add a new component for comparison or overrule a 
standard in any case, but rather clarified or added depth to the component being analyzed (e.g. Section 4.1.3.2 Table 11). In addition to identifying content necessary for comparison, each element needed to be assessed to determine whether the content within the matrix should be: a) compared with caution, and b) crucial for comparison, or c) irrelevant for comparison.

ISO 14025 Section 6.7.2, Requirements for comparability, states that Type III environmental declarations shall be deemed to have achieved comparability when a list of conditions has been met. This list includes conditions that are required to be equivalent, and conditions required to be identical. This was directly implemented in the chart to inform whether a section should be compared with caution, crucial for comparison, or is irrelevant towards comparison. One major deficiency in ISO 14025 is that equivalency is not defined, and therefore it is up to the user to decide whether the information is equivalent or not. For the purposes of this comparison, equivalence is defined as statements with the same scope and fulfill the same purpose or goal.

Crucial elements are those that if not present, not adequately defined, or accounted for differently preclude the comparison of products using the EPDs. Typically, these are those that are required to be identical as per ISO 14025. An example of this is the functional/declared unit, the unit used to quantify the environmental impacts of a product in the LCA, such as $1 \mathrm{~m}^{2}$ of a flooring product. However, this is not always the case; where deviations are proposed, this is discussed.

Elements requiring caution are those where there is the potential for errors to be introduced into the comparison. For example, impact category values that use different characterization factors ( $\mathrm{mol} \mathrm{H}+$ eq as opposed to $\mathrm{kg} \mathrm{SO}_{2} \mathrm{eq}$ ) that require manipulation or conversion could introduce error into the comparison.

Elements that are irrelevant are those that have no effect on the results or the validity of the comparison between products. These are included in the EPD Comparison Matrix as they can provide insight on the degree of compliance with ISO 14025 and other governing standards. Examples of this is the notes section, which is a cell for additional information pertaining to the EPD that does not fit into any other categories within the EPD Comparison Matrix. 


\subsubsection{Declaration Information}

\begin{tabular}{|c|c|c|c|c|c|c|c|c|}
\hline & & & & & & \multicolumn{3}{|c|}{ Governing Standards } \\
\hline & $\begin{array}{c}\text { Product } \\
\text { Name }\end{array}$ & $\begin{array}{c}\text { Declaration } \\
\text { Holder }\end{array}$ & $\begin{array}{c}\text { Programme } \\
\text { Operator }\end{array}$ & $\begin{array}{c}\text { Country } \\
\text { of } \\
\text { Origin }\end{array}$ & $\begin{array}{c}\text { Product } \\
\text { Category } \\
\text { Rules }\end{array}$ & $\begin{array}{c}\text { ISO } \\
14025\end{array}$ & $\begin{array}{c}\text { ISO } \\
21930\end{array}$ & $\begin{array}{c}\text { EN } \\
15804\end{array}$ \\
\hline STONE & $\begin{array}{l}\text { Rolan } \\
\text { Rockwool } \\
\text { Insulation } \\
\text { Board }\end{array}$ & Rolan & $\begin{array}{l}\text { International } \\
\text { EPD System }\end{array}$ & Mexico & $\begin{array}{l}2012.01 \\
\text { Version } 1.2 \text { for } \\
\text { Construction } \\
\text { Products }\end{array}$ & & & \\
\hline WOOL & $\begin{array}{l}\text { Mineral } \\
\text { Wool } \\
\text { Board }\end{array}$ & $\begin{array}{l}\text { North American } \\
\text { Insulation } \\
\text { Manufacturers } \\
\text { Association }\end{array}$ & $\begin{array}{l}\text { UL } \\
\text { Environment }\end{array}$ & USA & $\begin{array}{l}\text { Building } \\
\text { Envelope } \\
\text { Thermal } \\
\text { Insulation v1.2 }\end{array}$ & & & \\
\hline
\end{tabular}

Table 5: EPD Comparison Matrix Declaration Information Inputs

The information within the declaration information section is general and introductory. Inputs in this section include: a) Product Name, b) Declaration Holder (the company/product association who manufactures the product), c) Program Operator, d) Country of Origin (of product), e) Product Category Rules, and f) the governing standards that the EPD is generated under. These include standards ISO 14025, ISO 21930, and EN 15804.

\subsubsection{General Information}

ISO 14025 7.2, Declaration content, outlines parameters that must be included in a Type III EPD as identified in the PCR provided by the program operator. In the EPD Comparison Matrix, these parameters are separated into two sections: a) must be included, not crucial for comparison, and b) must be included, crucial for comparison. The separation is generated from the difference between information that would affect an EPD's completeness (caution), as opposed to information that would jeopardize a comparison between two or more EPDs (crucial). These parameters and their separation are displayed in Table 7. 

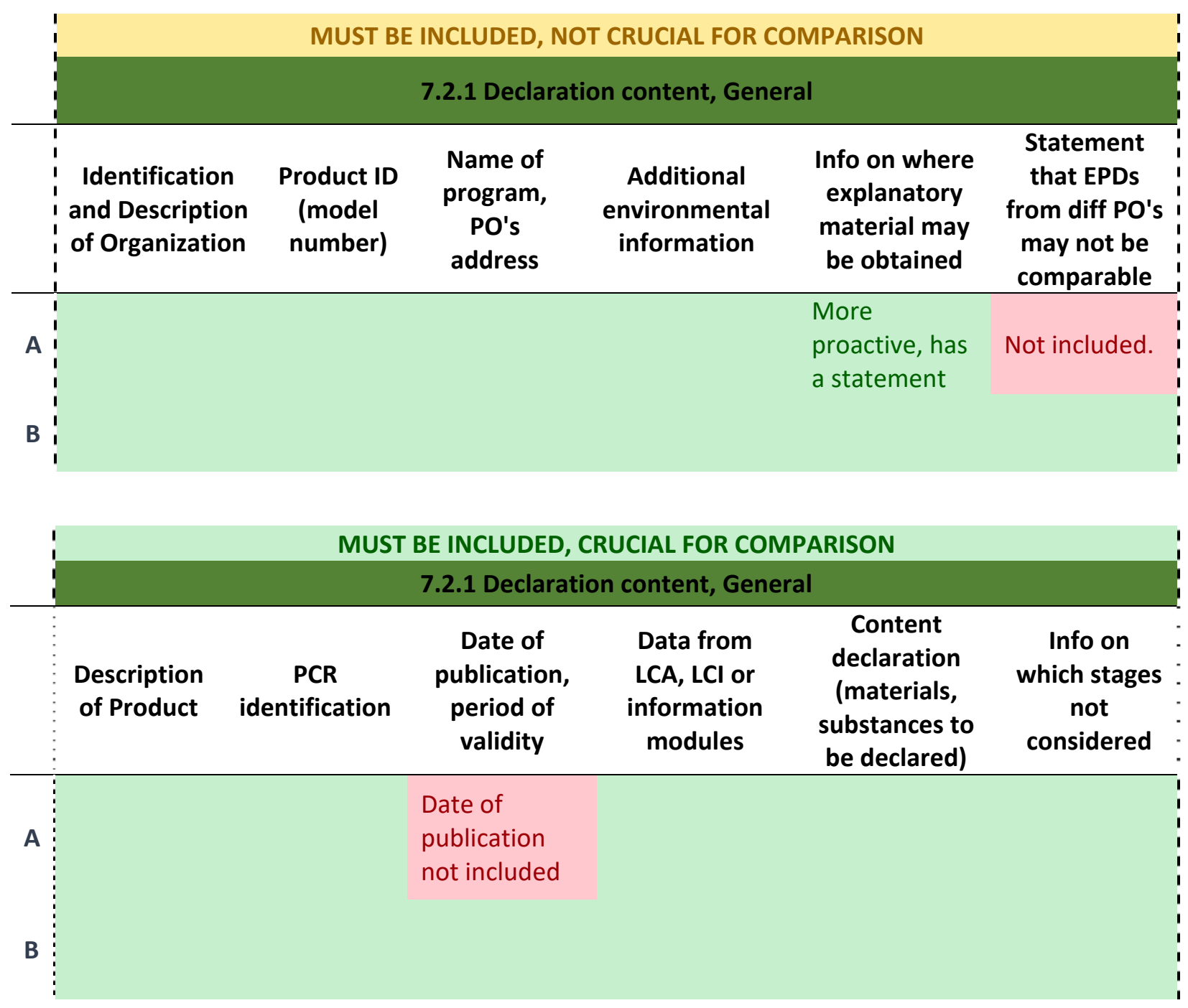

Table 6: EPD Comparison Matrix General Information Inputs

Within the EPD Comparison Matrix, column a) is to be compared with caution, and column b) is crucial for comparison. This separation does not exist in the standard, but has been adapted in the EPD Comparison Matrix. All items in column a) are those that are secondary to the comparison of two different EPDs. They may be important to the strength of a declaration, but have no effect when comparing two products. However, items in column b) have a direct correlation to the comparability of EPDs, as the items are focused on components that either describe the product in detail, the validity of the declaration, or the inclusion of vital material and environmental information within the declaration. For each column, the EPD is required to have all of the items within the list to be deemed comparable. 


\begin{tabular}{ll}
\multicolumn{1}{c}{$\begin{array}{c}\text { a) Must be included, not crucial for } \\
\text { comparison }\end{array}$} & \multicolumn{1}{c}{ b) Must be included, crucial for } \\
comparison
\end{tabular}

Table 7: Necessary declaration content from ISO 140257.2 with adaptation

\subsubsection{Goal and Scope Definition for LCA}

ISO 14025 6.7.2 b) Requirements for comparability, sets the goal and scope definition for the LCA of the product according to ISO 14040. This includes the following stipulations for identicalness and equivalency.

- The functional unit is identical;

- The system boundary is equivalent;

- The description of data is equivalent;

- The criteria for the inclusion of inputs and outputs are identical, and;

- The units are identical.

\subsubsection{Functional Unit}

The functional unit is defined as the "quantified performance of a product system for use as a reference unit" (ISO 14025). For example, a functional unit for carpeting could be stated as "1 square meter of installed flooring for a building". In the EPD Comparison Matrix, the functional unit is required to be identical and is crucial for comparison. Additionally, a declared unit (used when the LCA does not cover the entire life cycle, or when the exact function of the product in a building context is unknown) from one product that matches a functional unit from another product is deemed to be identical, and therefore comparable. 


\section{IDENTICAL}

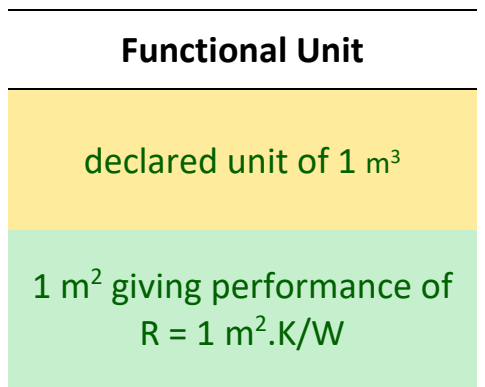

Table 8: EPD Comparison Matrix Functional Unit Inputs

Conversely, different functional units that cannot be easily converted - such as the example presented in Table 8 (volume determined by performance vs volume), are not directly comparable.

\subsubsection{System Boundaries}

\section{EQUIVALENT}

System Boundaries (ISO 14044) $\quad$ (MND = module not declared, MNR = module not relevant)

\begin{tabular}{|c|c|c|c|c|c|c|c|}
\hline 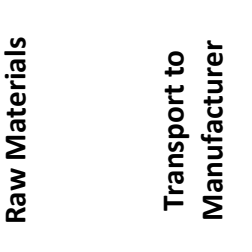 & 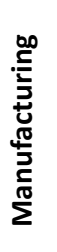 & 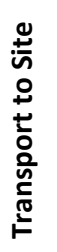 & 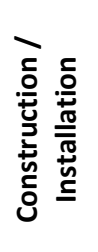 & 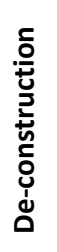 & 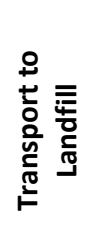 & 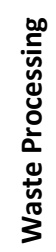 & 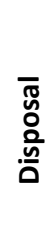 \\
\hline
\end{tabular}

\begin{tabular}{|c|c|c|c|c|c|c|c|c|c|}
\hline$x$ & $x$ & $x$ & $x$ & $x$ & $x$ & $x$ & $x$ & $x$ & MND \\
\hline$x$ & $x$ & $x$ & $x$ & $x$ & $x$ & $\begin{array}{c}x \\
20 \text { miles }\end{array}$ & $x$ & $x$ & MND \\
\hline
\end{tabular}

Table 9: EPD Comparison Matrix System Boundaries First Inputs

The system boundaries are the manufacturing processes that make up a product's LCA. System boundaries that should be included in an EPD for equivalency are not included in ISO 14025 , and are only illustrated as an example in ISO 21930. The system boundaries included in the EPD Comparison Matrix are a simplified version of the parameters mandated in EN 15804 6.4.3 System Boundaries: 


\begin{tabular}{|l|l|}
\hline Raw Material Acquisition (A1) & Refurbishment (B5) \\
\hline Transport to Manufacturer (A2) & Operational Water Use (B6) \\
\hline Manufacturing (A3) & Operational Energy Use (B7) \\
\hline Transport to Site (A4) & De-construction/Demolition (C1) \\
\hline Construction/Installation (A5) & Transport to Landfill (C2) \\
\hline Use (B1) & Waste Processing (C3) \\
\hline Maintenance (B2) & Disposal (C4) \\
\hline Repair (B3) & $\begin{array}{l}\text { Reuse-Recovery-Recycling Potential beyond } \\
\text { the product system boundary (D1) }\end{array}$ \\
\hline
\end{tabular}

Table 10: System Boundaries Included in EPD Comparison Matrix

Also included as part of this section are two columns, neither of which are included in any of the standards, but are components deemed necessary for fair comparison. This is illustrated in Table 11 and described below.

\begin{tabular}{|c|c|}
\hline IDENTICAL & IDENTICAL \\
\hline $\begin{array}{l}\text { Are Impacts Separated by } \\
\text { System Boundary Modules? }\end{array}$ & $\begin{array}{l}\text { Are System Boundaries the exact } \\
\text { same? }\end{array}$ \\
\hline NO & N/A \\
\hline YES & N/A \\
\hline
\end{tabular}

Table 11: EPD Comparison Matrix System Boundaries Second Inputs

The first column is the separation of LCA environmental impact data reporting by system boundary in the EPD. EPDs will report the environmental impact data in one of three ways, in order of granularity: as a combined total, per module (product stage, use stage, end-of-life stage) or per life cycle stage (raw material acquisition, transport to manufacturer, manufacturing, etc). This column in the EPD Comparison Matrix asks if the impacts are separated, at the very least, by life cycle modules. As per EN 15804 Section 6.4.3 System Boundaries, the modules are as follows:

- A1-A3: Product stage;

- A4-A5: Construction stage;

- B1-B7: Use stage; 
- C1-C4: End-of-life stage;

- D: Reuse-Recovery-Recycling Potential beyond the product system boundary.

A "yes" is inserted if the EPD separates the environmental impacts of the product, at the very least, per module (EPDs separated per life cycle stage also qualify for "yes"s). A "no" is inserted if the EPD declares the environmental impacts of the product as a total.

The second column asks if the system boundaries used are identical. This column is not filled out in the Inputs section, but is here because the column is asking for information to be completed within the Comparison section (i.e. the column asks for information related to comparison, not a singular EPD).

\subsubsection{Description of Data}

Description of data is a discussion that must be present in every EPD, describing which processes in the LCA used specific data, and which used generic data. EN 15804 6.3.6 Selection of Data states that as a general rule, specific data shall be the first choice for calculating an EPD, and an EPD describing a specific product shall be calculated using specific data for at least the processes the manufacturer has direct control over. In most cases, this is only the manufacturing stage. In the EPD Comparison Matrix, each system boundary described in Section 4.1.3.2 is repeated. Rows are filled out by describing the products use of specific or generic data for each system boundary. A sample table from the EPD Comparison Matrix is shown in Table 12. 


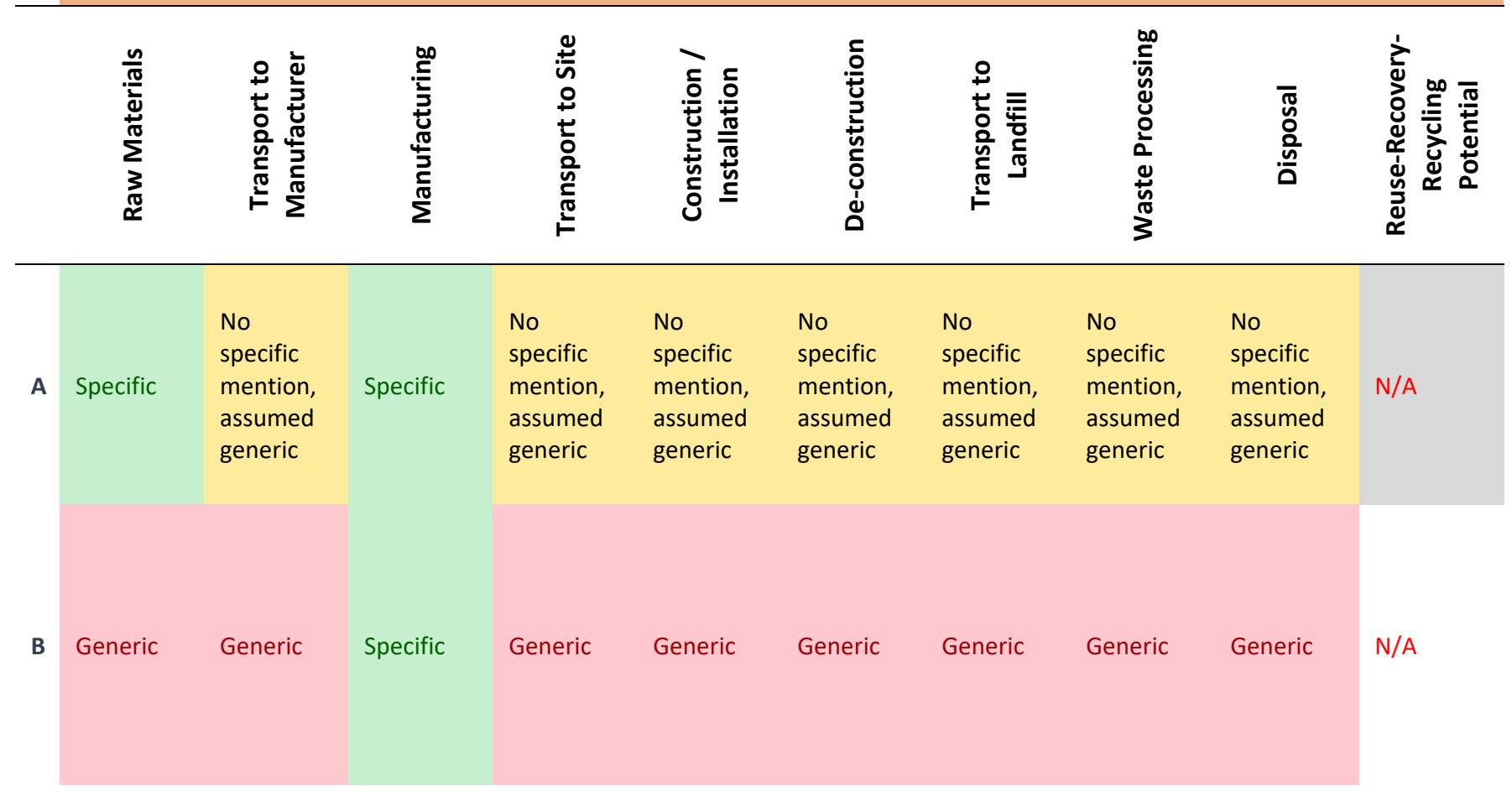

Table 12: EPD Comparison Matrix Description of Data Inputs

\subsubsection{Criteria for the Inclusion of Inputs and Outputs}

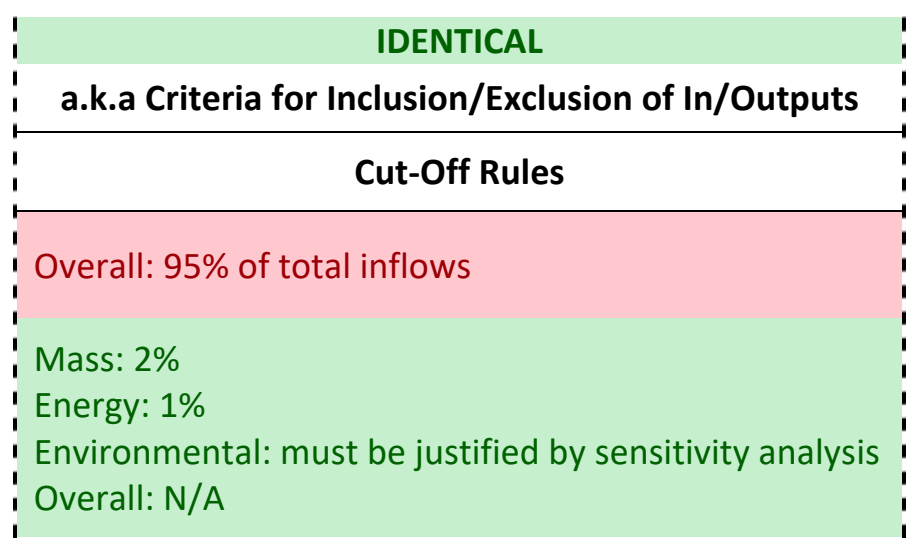

Table 13: EPD Comparison Matrix Cut-off Rules Inputs

Another name for the criteria for the inclusion of inputs and outputs is "cut-off rules". It describes the amount or percentage of data that is allowed to be excluded from the LCA process due to undesired complexity, insufficient input data gaps, or data gaps for unit processes. Cut-off rules are usually expressed for the following characteristics: the amount of mass input that can be excluded, the amount of energy input that can be excluded, the amount of environmental output that can be excluded, and an overall total input/output (the addition of mass and energy) that 
can be excluded. When comparing two EPDs, cut-off rules must be identical for all the characteristics listed to allow product comparison.

\subsubsection{Units}

EQUIVALENT

Table 14: EPD Comparison Matrix Units Inputs

The input for the Units column is either SI or IP, depending on the units used for the EPD. Units must be identical between two EPDs for direct comparison. However, the conversion of a value from metric to imperial measuring systems, or vice versa, is not deemed as critical for comparison in the EPD Comparison Matrix, and is acceptable to allow product comparison but requires caution.

\subsubsection{Impact Categories}

According to ISO 21930 8.2.2 Declaration of environmental impacts, use of resources and generation of waste, the following impact categories are to be included in the EPD.

Inputs in this section include the indicators used for each impact category. Both the impact categories and their indicators must be equivalent when comparing two EPDs. This section is illustrated in Table 14.

In cases where an impact category is identified using different characterization factors, like eutrophication with $\mathrm{kg} \mathrm{N}$ eq or $\mathrm{kg} \mathrm{PO}_{4}{ }^{3-}$ eq (nitrogen or phosphate equivalents), and acidification with $\mathrm{kg} \mathrm{SO}_{2}$ eq or mol $\mathrm{H}+$ eq (sulphur dioxide or hydrogen ion equivalents), conversion ratios were developed to aid comparison where EPDs state impacts differently. The conversion ratios are explained in each section where appropriate. These become important in the Impact Category Results portion of the EPD Comparison Matrix, which is explained in Section 4.4.

\subsubsection{Global Warming Potential}

Global warming potential, often described as greenhouse gases or climate change, is a global environmental impact. Its main function is a warming effect caused by $\mathrm{CO}_{2}$ (carbon dioxide) and 
other greenhouse gases in the atmosphere (DMotE, 2005), identified using $\mathrm{kg} \mathrm{CO}_{2}$ eq within EPDs.

\subsubsection{Ozone Deplion}

Depletion of the stratospheric ozone layer is a global environmental impact. It is caused by the release of substances that deplete and breakdown ozone $\left(\mathrm{O}_{3}\right)$ (DMotE, 2005). Ozone depletion is identified using trichlorofluoromethane (kg CFC-11 eq, sometimes written as R-11).

\subsubsection{Acidification}

Acidification is a regional and local environmental impact. It is defined as the releases of protons in the terrestrial or aquatic systems leading to increased mobility of heavy metals and aluminium, contributing to acid rain (DMotE, 2005). Acidification is identified using either sulfur dioxide $\left(\mathrm{kg} \mathrm{SO}_{2} \mathrm{eq}\right)$ or hydrogen ion $(\mathrm{mol} \mathrm{H}+\mathrm{eq})$ equivalents.

A conversion between $\mathrm{kg} \mathrm{SO}_{2}$ eq and $\mathrm{mol} \mathrm{H}+$ eq for the purposes of comparing EPDs that declare acidification in varying ways. To convert from $\mathrm{mol} \mathrm{H}+$ eq to $\mathrm{kg} \mathrm{SO}_{2} \mathrm{eq}$, the value should be multiplied by 32 . To convert from $\mathrm{kg} \mathrm{SO}_{2}$ eq to $\mathrm{mol} \mathrm{H}+$ eq, the value should be divided by 32 , i.e. $1 \mathrm{~kg} \mathrm{SO} 2=1 \mathrm{~mol} \mathrm{H}+/ 0.032$.

$$
\begin{gathered}
\mathrm{SO}_{2}+0.5 \mathrm{O}_{2}+\mathrm{H}_{2} \mathrm{O}=\mathrm{H}_{2} \mathrm{SO}_{4} \\
\mathrm{SO}_{2}->32+16 \times 2=64 \mathrm{~g} / \mathrm{mol} \\
32 \mathrm{~g} \mathrm{SO}_{2} / \mathrm{mol} \mathrm{H}+ \\
0.032 \mathrm{~kg} \mathrm{SO}_{2} / \mathrm{mol} \mathrm{H}+
\end{gathered}
$$

Equation 1: Acidification Conversion Process

\subsubsection{Eutrophication}

Eutrophication, or nutrient enrichment, is a regional and local environmental impact.

Eutrophication is the enrichment of water bodies with nutrient salts that leads to increased algae, plankton, and aquatic plant growth (DMotE, 2005), and is identified using either nitrogen (kg N eq) or phosphate (kg PO${ }_{4}^{3-}$ eq) equivalents.

A conversion between $\mathrm{kg} \mathrm{N}$ eq and $\mathrm{kg} \mathrm{PO}_{4}{ }^{3-}$ eq has been developed for the purposes of comparing EPDs that declare eutrophication differently. This conversion uses a basis of $100 \mathrm{~g}$ of algae biomass, which contains $12.2 \mathrm{~g}$ of $\mathrm{N}$ (nitrogen) and $2.3 \mathrm{~g}$ of $\mathrm{P}$ (phosphorus). This gives an $\mathrm{N}: \mathrm{P}$ ratio of 5.3:1 in the biomass, which serve as limiting reagents. The resulting eutrophication impact conversion between these two characterization factors are: 
$100 \mathrm{~g}$ algae biomass $=12.2 \mathrm{~g} \mathrm{~N}+2.3 \mathrm{~g} \mathrm{P}+85.5 \mathrm{~g}$ other

$$
\begin{gathered}
12.2 \mathrm{~g} \mathrm{~N} / 2.3 \mathrm{~g} \mathrm{P}=5.3 \\
1 \mathrm{~kg} \mathrm{PO}{ }^{3-} \text { eq }=0.1887 \mathrm{~kg} \mathrm{~N} \text { eq } \\
1 \mathrm{~kg} \mathrm{~N} \text { eq }=5.3 \mathrm{~kg} \mathrm{PO}_{4}{ }^{3-} \text { eq } \\
\text { Equation 2: Eutrophication Conversion Process }
\end{gathered}
$$

\subsubsection{Formation of Tropospheric Ozone}

Formation of tropospheric ozone, which is sometimes referred to photochemical ozone creation potential (POCP) or smog potential, is a regional and local environmental impact. Essentially, this impact is the development of ground-level ozone. It is identified using $\mathrm{kg} \mathrm{C}_{2} \mathrm{H}_{4} \mathrm{eq}$ (ethylene), kg NOx (mono-nitrogen oxides), kg O3 eq (ozone), and kg NMVOC eq (nonmethane volatile organic compounds).

Due to the complexities of atmospheric chemistry, conversion between different characterization factors is not recommended. Further, normalization and weighting differs substantially across regions (DMotE, 2005) further complicating any comparison.

Within this thesis, a difference in ozone formation characterization factors will be deemed grounds for non-comparison of EPDs.

\subsubsection{Depletion of Energy and Materials}

This category comprises the following environmental impacts:

- Depletion of non-renewable energy resources, identified using MJ;

- Depletion of non-renewable material resources, identified using MJ;

- Use of renewable primary energy, identified using MJ;

- Use of renewable material resources, identified using MJ;

\subsubsection{Consumption of Freshwater}

Consumption of freshwater quantifies the amount of water used in the cradle-to-grave lifecycle, identified using $\mathrm{m}^{3}$.

\subsubsection{Waste}

There are two Waste categories in an EPD:

- Hazardous waste, the total amount of waste that can potentially cause harm to human health and the environment (ISO 14025), identified using $\mathrm{kg}$, and;

- Non-hazardous waste, all other waste, identified using kg. 


\subsubsection{Not Converted}

This column inquires whether or not any of the system boundaries had to be converted. This adds a level of caution to the comparison of EPDs. This is explained further in Section 4.2.4 and 4.3.2.4.

\subsubsection{Other Inputs}

EQUIVALENT EQUIVALENT

\begin{tabular}{|c|c|c|c|c|c|c|}
\hline & $\begin{array}{l}\text { Period of } \\
\text { Validity }\end{array}$ & $\begin{array}{l}\text { Service Life } \\
\text { Time Period }\end{array}$ & $\begin{array}{c}\text { LCA } \\
\text { Methodology }\end{array}$ & $\begin{array}{c}\text { Mentions of } \\
\text { Comparability }\end{array}$ & File Path & NOTES \\
\hline A & 3 years & 60 years & $\begin{array}{l}\text { Does not } \\
\text { exactly specify }\end{array}$ & $\begin{array}{l}\text { Does not } \\
\text { mention } \\
\text { comparability }\end{array}$ & 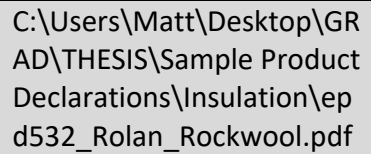 & \\
\hline B & 5 years & 60 years & GaBi 4 & Mentioned & 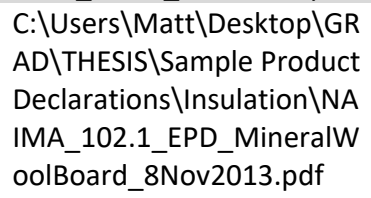 & \\
\hline
\end{tabular}

Table 15: EPD Comparison Matrix Other Inputs

Included in this section are items that do not fit into other sections but are still required to be in an EPD. According to ISO 14025 6.7.2 Requirements for comparability, mentions of comparability to other EPDs is required to be included (covered in Section 4.1.2). However, in the EPD Comparison Matrix, this is not included as a requirement for comparison; that while this is mandatory for inclusion in the EPD, it becomes redundant and can possibly exclude a comparison between products where not necessary and has no effect on comparability. Given the lack of EPDs available in North America, excluding products based on comparability statements is not ideal.

Additionally, the period of validity (their mandatory inclusion also covered in Section 4.1.2) are required to be equivalent.

There are no requirements for the LCA methodology in any international standard governing the creation or use of EPDs, and is only included as an item of due diligence. The remaining columns, which include the File Path and Notes, are included for the ease of the user.

\subsection{Environmental Product Declaration Comparison}

This section of the chart uses the information from the Inputs section for factorial comparisons. in a factorial fashion. Every EPD is assigned a letter $(A, B, C \ldots)$ and is then compared to every 
other EPD in the chart (A vs B, A vs C, B vs C...). Every possible comparison within the matrix is included in this section.

The comparison is populated with either a "yes" or "no", depending on the section it is being used in. A "yes" constitutes that the EPD discloses information in the same way, and a "no" means either the two EPDs don't disclose information in the same way, or one EPD does not disclose it in an appropriate way. This definition alters depending on which section from the Inputs is being analyzed. An example of the Comparison section is shown in Table 17.

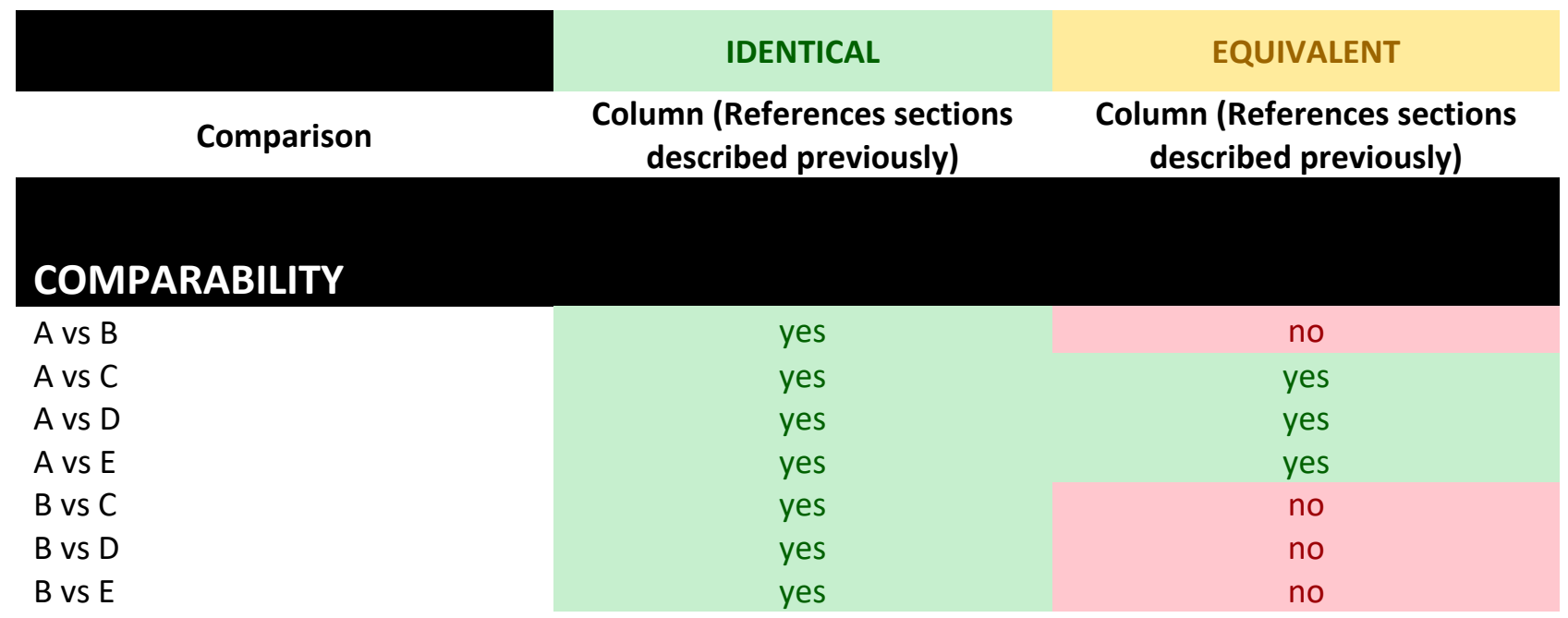

Table 16: EPD Comparison Matrix Comparison Section

This Comparison section is organized in the same fashion as the previous section, 5.1 Inputs, as it is a reflection of that part of the matrix.

\subsubsection{Declaration Information}

Two comparisons are completed in this section. One comparison is whether the PCR is the same; a "yes" is used if they are both identical, a "no" is used if they are not. The second comparison focuses on the governing standards of the EPD, specifically ISO 14025 and EN 15804. A "yes" is used if they both use the standard; a "no" is used if one or both of the EPDs being compared does not use the standard.

\subsubsection{General Information}

All columns within the general information category are compared. A "yes" value is assigned if both EPDs disclose the information required by ISO 14025, while "no" is assigned if one of both of the EPDs does not disclose the required information. 


\subsubsection{Goal and Scope Definition of LCA}

This section will explain all the comparisons in the Goal and Scope Definition of LCA section, broken down into subsection similar to Section 3.1.3.

\subsubsection{Functional Unit}

The Functional Unit section only consists of one column, and therefore there is only one comparison. A "yes" is used if the functional or declared units in the EPD are exactly identical, or able to be divided to be identical. For example, an EPD that has a functional unit of $1 \mathrm{~m}^{2}$ of floor covering would be identical to an EPD with $1000 \mathrm{~m}^{2}$ of floor covering. A "no" is used if they are not identical or not divisible.

\subsubsection{System Boundaries}

The System Boundaries section contains eighteen columns, all of which are used for comparison. The first sixteen columns in this section are typical system boundaries that could possibly be found in an EPD for a construction product. These system boundaries are described in Section 4.2.3.2. A "yes" is inserted into the cell corresponding to the system boundary being analyzed if it is declared in both EPDs in a similar function. For example, if both EPDs declare Raw Material Acquisition, a "yes" is inserted in that column. A "yes" is also inserted if both EPDs do not include the system boundary. Both of these factors constitute an equivalent level of comparison. A "no" is inserted if the system boundary is not included in one of the two EPDs being compared. Table 18 below provides an illustrative example of how this section works.

$\begin{array}{cccc}\text { EPD Inputs } & \text { Raw Material Acquisition } & \text { Transport to Manufacturer } & \text { Manufacturing } \\ \text { A } & \text { No } & \text { Yes } & \text { Yes } \\ \text { B } & \text { Yes } & \text { Yes } & \text { No } \\ \text { C } & \text { Yes } & \text { Yes } & \text { No } \\ \text { Comparison } & \text { No } & \text { Yes } & \text { No } \\ \text { A vs B } & \text { No } & \text { Yes } & \text { Yes } \\ \text { A vs C } & \text { Yes } & \text { Yes } & \\ \text { B vs C } & \text { Table 17: System Boundaries Comparison Section Illustrative Example } & \\ \text { The last two columns in the System Boundaries section, titled "Are Impacts Separated by } & \\ \text { System Boundaries?" and "Are System Boundaries the Exact Same?" also require inputs. In the } \\ \text { first column, a "yes" is inserted if both EPDs separate system boundaries by life cycle module. A } \\ \text { "no" is inserted if one or both EPDs being compared does not separate the data by life cycle } \\ \text { module. In the second column, a "yes" is inserted if both products being compared declare the }\end{array}$


exact same system boundaries. A "no" is inserted if they both use different system boundaries in the EPD.

\subsubsection{Description of Data}

The Description of Data Comparison section asks if the two EPDs used specific data (as opposed to generic data) for all of the system boundaries included in the previous section. A "yes" is used if both EPDs being compared use specific data. A "no" is used if one or both of the EPDs being compared use generic data for the processes.

\subsubsection{Criteria for the Inclusion of Inputs and Outputs}

The criteria for the inclusion of inputs and outputs section asks if both EPDs have an identical set of cut-off rules. Because the information regarding the application of cut-off rules is insufficient in most EPDs, this was taken directly from the PCR.

Cut-off rules are stated in one of three ways in EPDs. One, they are not included in the EPD and must be sourced from the PCR, two, are listed in the EPD, but the application of the cut-off rules in the LCA of the product is not described (i.e. were cut-off rules used or not used in the LCA, and if they were used, what processes were excluded from the LCA?) or three, the EPD does not state what the cut-off rules were, but states that they were not applied. Only a small number of EPDs both list the cut-off rules and describe their application in the LCA.

In this section, a "yes" is used the guideline for the cut-off rules in both EPDs being compared are exactly the same. A "no" is used if the EPDs being compared use differing guidelines for the application of cut-off rules.

\subsubsection{Units}

EPDs must use the same units, or system of measurement, to enable comparison (SI vs IP). A "yes" is used if both EPDs being compared use the same system of measurement, a "no" is used if both EPDs being compared use differing systems of measurement. In this latter case, unit conversion can be used to allow comparison but is an element that requires caution when comparing.

\subsubsection{Impact Categories}

All columns in the Impact Categories Inputs section, described in Section 4.1.4, are used for comparison. This section asks if the environmental impact indicator (e.g. $\mathrm{kg} \mathrm{CO}_{2}$ eq for Global Warming Potential) used is the exact same in both EPDs being compared. 
A "yes" is used if both EPDs being compared use the exact same environmental impact indicator in each of the impact categories. A "yes" is also used if the EPDs use differing environmental impact indicators, but are easily convertible. An example of this would be two EPDs that declare freshwater use in cubed meters and gallons, respectively.

A "no" is used in two cases. In one, both EPDs being compared use different environmental impact indicators for one of the impact categories. In the other, one of the EPDs being compared does not declare one of the impact categories listed in Section 4.1.4.

Another column is included in this section, titled "Not Converted". It is inquiring whether or not there was a conversion of a characterization factor in the comparison. A "yes" is used if there were no conversions in any impact category in the two EPDs being compared. A "no" is used if one of the EPDs converted an impact to a different characterization factor.

\subsubsection{Other Inputs}

Two columns from the Other Inputs section are used in the Comparison section. First, the period of validity is compared. A "yes" is used if both EPDs have the same period of validity. A "no" is used if the period of validity in both EPDs being compared differ from one another. Second, the service life time period is compared. A "yes" is used if the EPDs being compared state an equivalent service life. A "no" is used if one or both of the EPDs being compared do not have an equivalent service life, do not disclose a quantified service life (i.e. as long as the lifetime of the building rather than 10 years, 50 years), or do not disclose a service life at all.

\subsection{Environmental Product Declaration Summarization}

This section will describe the Summarization process that is generated from the completion of the Comparison section in the EPD Comparison Matrix. This process either reflects the cells in the Comparisons section, or conglomerates inputs across columns to provide clearer results. EPDs are then scored based on the results in this section.

The columns in this Summarization section, which will be elaborated on in Sections 4.3.1 and 4.3.2, and the rationale for why each element has been assigned to the crucial or cautious category, are shown in Table 19. 


\begin{tabular}{|c|c|}
\hline Crucial Cates & Cautious Category (refer to 4.3.2) \\
\hline $\begin{array}{l}\text { Both EPDs have a Functional/Declared } \\
\text { Unit which is the same, or easily } \\
\text { convertible } \\
\text { - this is necessary to define the quantity } \\
\text { of material being compared and thus } \\
\text { determine whether a common basis can } \\
\text { be found for comparison between } \\
\text { EPDs. } \\
\text { Both EPDs either: } \\
\text { a) Separate their system } \\
\quad \text { boundaries by life cycle module, } \\
\quad \text { or; } \\
\quad \text { b) Use the exact same system } \\
\quad \text { boundaries } \\
\text { - this is necessary to allow the } \\
\text { products to be compared for the } \\
\text { same lifecycle elements. } \\
\text { Both EPDs' Impact Category } \\
\text { characterization factors consistently the } \\
\text { same, or easily convertible - this is } \\
\text { necessary to allow like-for-like } \\
\text { comparison } \\
\text { Both EPDs include all mandatory } \\
\text { content required for comparison - this } \\
\text { ensures that the minimum ISO } 14025 \\
\text { requirements are met and that it is clear } \\
\text { to the designer what is covered by the } \\
\text { EPD. } \\
\text { Both EPDs use same cut-off rules - this } \\
\text { ensures that there is consistency in cut- } \\
\text { off rules for both products. }\end{array}$ & $\begin{array}{l}\text { Both EPDs are created using same Product } \\
\text { Category Rules - different PCRs could result in } \\
\text { different definitions and interpretations of reported } \\
\text { values. } \\
\text { Both EPDs adhere to the same standards - EPDs } \\
\text { using different standards could result in different } \\
\text { requirements or formatting for declarations } \\
\text { Both EPDs include all mandatory content not } \\
\text { necessarily required for comparison - EPDs which } \\
\text { do not include all mandatory content may be an } \\
\text { indicator of poor quality EPDs } \\
\text { Both EPDs use a different set of system } \\
\text { boundaries up to Reuse-Recovery-Recycling } \\
\text { Potential - EPDs need to compared with caution if } \\
\text { they declare environmental impacts for different life } \\
\text { cycle stages } \\
\text { Both EPDs include Reuse-Recovery-Recycling } \\
\text { Potential - largely a positive environmental impact, } \\
\text { EPDs need to be compared with caution if one } \\
\text { does not declare the boundary } \\
\text { Both EPDs use specific data for the Manufacturing } \\
\text { system boundary - specific data is much more } \\
\text { accurate than generic data for processes within } \\
\text { manufacturer's control } \\
\text { Both EPDs use the same system of measurement, } \\
\text { units (IP v. SI) - results from EPDs need to be } \\
\text { converted if different } \\
\text { Both EPDs include: } \\
\text { a) Period of validity/date of expiration } \\
\text { - EPDs which do not include these contents } \\
\text { may be an indicator of poor quality EPDs } \\
\text { c) LCA Methodology } \\
\text { d) Statement of Comparability }\end{array}$ \\
\hline
\end{tabular}

Table 18: Summarization Factors in EPD Comparison Matrix

Essentially, the comparison between two EPDs cannot be done if there is a "no" present in any of the crucial categories. The crucial categories provide a level of caution that should be 
implemented when using EPDs to directly compare products. This section is entirely automated, according to the cell values present in the Comparison section. A sample of this component of the matrix is shown on the following page in Table 20.

\subsubsection{Crucial Categories}

\subsubsection{Same Functional/Declared Unit}

This column inquires whether or not the functional or declared units in both EPDs being compared are the same, or are convertible. This is described in full in Sections 4.1.3.1 and 4.2.3.1. The functional/declared unit is required to be identical in ISO 14025, and can greatly affect the comparison process. For example, a comparison of insulation, where one EPD uses 1 $\mathrm{m}^{2}$ of insulation material with a thickness that gives a design thermal resistance of $1 \mathrm{~m}^{2} \cdot \mathrm{K} / \mathrm{W}$, and the other which uses $1 \mathrm{~m}^{3}$ of insulation material, cannot be compared (without the addition of supplementary material to convert, not available in many cases).

A "yes" is automatically inserted if the functional or declared units in both EPDs are the same or convertible, a "no" is automatically inserted if the functional or declared units are not the same.

\subsubsection{System Boundaries Separated or Same}

This column uses the results from two columns in the Comparisons chart, both described in Sections 4.1.3.2 and 4.2.3.2. These columns include: whether both EPDs being compared separate their system boundaries by life cycle module (Column A), and if both EPDs being compared use the same exact set of system boundaries (Column B). The reasoning for this is two-fold: an appropriate level of comparison requires that system boundaries are separated by life cycle module, if one of two EPDs do not declare the same system boundaries, a comparison can still be done using the ones included. If they are not separated, the comparison becomes nearly impossible. Additionally, a comparison can be done if the set of system boundaries used between two EPDs are the exact same, even if they are not separated. Under this definition, two EPDs cannot be compared if one of two EPDs being compared do not separate the system boundaries by life cycle module and uses a different set of system boundaries than the other.

As explained in Sections 4.1.3.2 and 4.2.3.2, these exact stipulations are not included in any of the standards used to build the matrix. However, it complements ISO 14025's requirements for the system boundaries to be identical for comparison. 


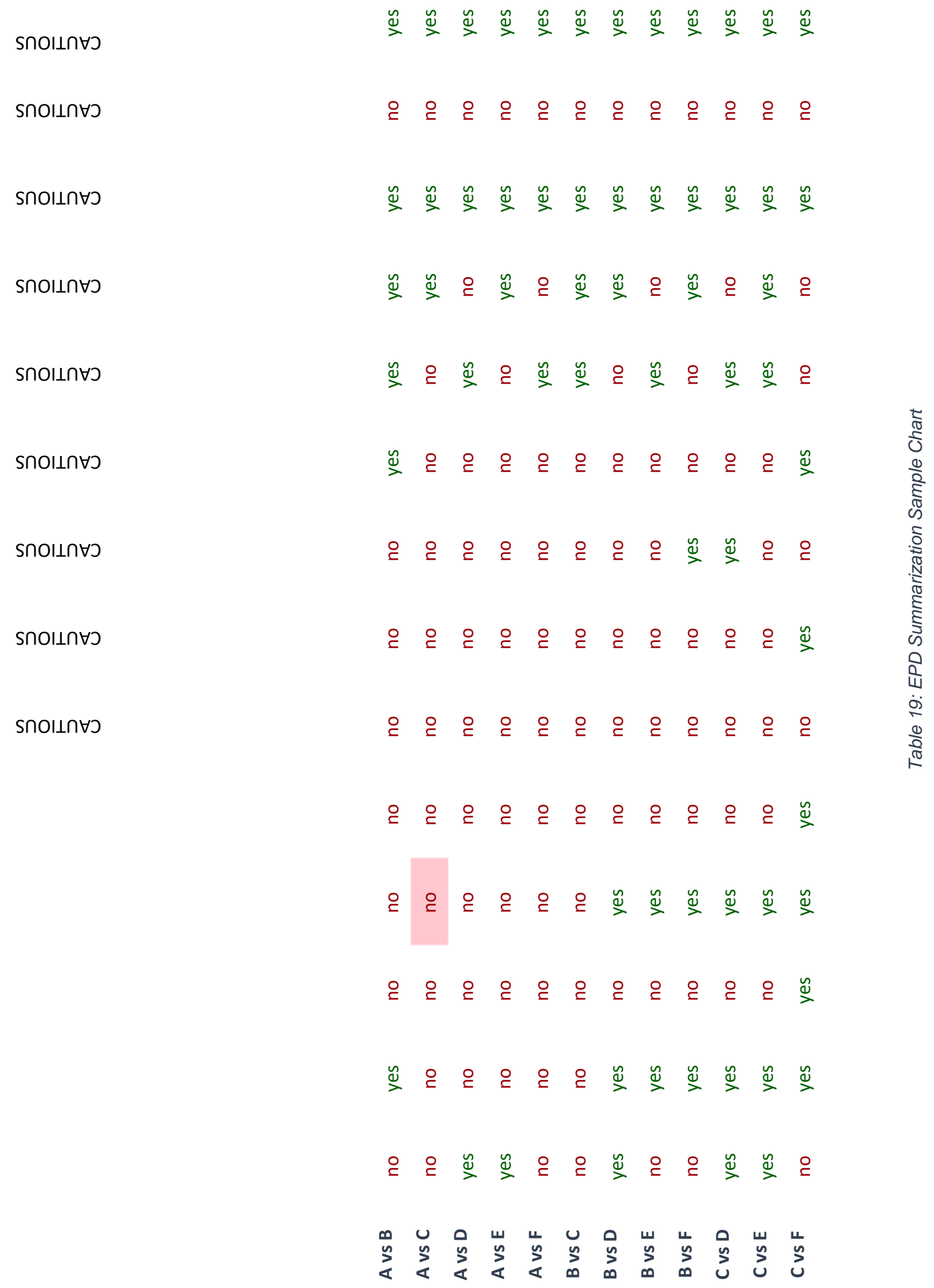


This column uses the results from Column A and Column B in an and/or scenario. A "yes" is automatically inserted if either Column A, Column B, or both have a "yes" in the comparison chart. A "no" is automatically inserted if both Column A and Column B have a "no" in the comparison chart.

\subsubsection{Impact Category Characterization Factors}

This column, focusing on the Impact Categories section outlined in 5.1.4, summarizes the Comparison results described in Section 4.2 .4 by using all columns. EPDs are not comparable if the environmental impacts are declared in units that are not easily convertible to match (such as in the case of atmospheric environmental impacts, described in Section 4.1.4.5), and are required to be identical in ISO 14025.

A "yes" is used if both EPDs being compared declare their environmental impacts using all the same characterization factors. If any of the characterization factors are different and they are easily convertible, this also contributes toward a "yes". A "no" is automatically inserted if one or multiple of the characterization factors used in both EPDs are different and not easily convertible.

\subsubsection{Mandatory Content for Comparison}

This column summarizes the elements in Section 4.1.2 Table 7 Column b). A "yes" is automatically inserted here if all of the elements are included in both EPDs, including: description of the product, PCR identification, date of publication and period of validity, data from LCA, LCl, or information modules, content declaration, and information on which life cycle stages are not considered. A "no" is automatically inserted if one of or multiple elements are not present in either of the EPDs being compared.

\subsubsection{Cut-Off Rules}

This column directly reflects the cut-off results column in the Inputs and Summarization components of the EPD Comparison Matrix, described in Section 4.1.3.4 and 4.2.3.4. Cut-off rules are required to be identical in ISO 14025, and are crucial for comparison because versions of cut-off rules in each PCR exclude different amounts of energy, mass, and environmental impact from LCA results.

A "yes" is automatically inserted if it is present in the Comparisons section, a "no" is used if it is not. 


\subsubsection{Cautious Categories}

\subsubsection{Same PCRs}

In reality, two EPDs that are being directly compared are required to have the same PCR. This ensures all provisions in ISO 14025 6.7.2 Requirements for comparability are met, and is a statement that is included in many EPDs. In this case, the EPD Comparison Matrix aims to compare EPDs no matter which PCR they used, and because of this, a comparison being done is only a component to be cautious about, not one to eliminate the possibility of comparison.

This column directly reflects the PCR column described in Sections 4.1.1 and 4.2.1. A "yes" is automatically inserted if both EPDs being compared have the same PCR. A "no" is automatically inserted if both EPDs being compared have differing PCRs.

\subsubsection{Same Standard Set}

This column determines whether or not both EPDs adhere to the exact same set of standards, namely ISO 14025 and EN 15804. This is referenced in Sections 4.1.1 and 4.2.1. This is a category that requires caution because it has overarching effects on the comparison results. A "yes" is automatically inserted if both EPDs have the same combinations of standards, either both ISO 14025, or both ISO 14025 and EN 15804. A "no" is automatically inserted if both EPDs are written using different sets of standards.

\subsubsection{Mandatory Content for Comparison}

This column summarized the elements in Section 4.1.2 Table 7 Column a). This is the opposite of the crucial category with the same title, the difference between the two is discussed in Section 4.3.1.4. A "yes" is automatically inserted if all elements are included in both EPDs, including: identification and description of the organization who holds the EPD, the product ID (model number or other similar identifier), the name of the PO and their address, the inclusion of additional environmental information, information on where explanatory material may be obtained, and a statement that EPDs from different POs may not be comparable. A "no" is automatically inserted if one of or multiple elements are not present in either of the EPDs being compared.

\subsubsection{System Boundaries up to Reuse-Recovery-Recycling Potential}

This column asks if all the system boundaries up to the Reuse-Recovery-Recycling (R-R-R) Potential system boundary are the same. The reasoning behind the separation between the $R$ $R-R$ Potential and the rest of the system boundaries is that this specific system boundary almost 
always contributes positively to the environmental impacts of the system, lessening the product's environmental impact. It is also not included in every EPD. So, the separation is needed to distinguish between the negative and positive environmental impacts of EPDs. This becomes increasingly important if the EPD does not separate its system boundaries by module, including R-R-R Potential in the total environmental impact, which can skew the numbers for a practitioner using EPDs who is not familiar with the LCA processes.

A "yes" is automatically generated if all system boundary columns described in Section 4.1.2.3 and 4.2.2.3 are the same for both EPDs being compared. A "no" is automatically generated if there are differences in one of the system boundary columns.

\subsubsection{Reuse-Recovery-Recycling Potential}

Reuse-Recovery-Recycling Potential is important on as a sole component based on the fact that the environmental impacts from this system boundary are always positive (i.e. they would contribute to a better environmental performance of a product). Continuing from the preceding section, a "yes" is automatically generated if both EPDs being compared either include or both exclude the R-R-R Potential system boundary. A "no" is automatically generated if one of the two EPDs being compared declares this system boundary, and the other does not.

\subsubsection{6. $\quad$ Specific Data for Manufacturing Process}

According to EN 15804 Section 6.3.6 Selection of data, specific data derived from processes in direct control of the manufacturer shall be the first choice as a basis for calculating an EPD. Not all EPDs follow the EN 15804 standard, but this is a good indicator of a comparable EPD, considering the exploration of specific as opposed to generic data described in Section 2.7. As the Manufacturing stage is the only system boundary that the manufacturer has direct control over in every scenario, it is the only one required to be generated from specific in this section.

A "yes" is automatically generated if both EPDs being compared use specific data for their Manufacturing system boundary. A "no" is automatically generated if one or both EPDs being compared do not use specific date for the Manufacturing system boundary.

\subsubsection{Units}

This section refers to whether the EPD was published using SI or IP units, or in some cases, both. A "yes" is automatically generated if the two EPDs being compared were published with the same units of measurement. A "no" is automatically generated if one of the EPDs being compared is published with different units of measurement than the other. 


\subsubsection{Inclusion of Other Inputs}

The Other Inputs section is described in detail in Sections 4.1.5 and 4.2.5. The elements in this section include: a statement of validity (date of publication, date of expiration, period of validity), and a reference service life for the product. These are components found in most EPDs, and a practitioner should be cautious of the quality of the EPD if either of these components are omitted in the comparison. A "yes" is automatically generated if both EPDs being compared contain both of these elements in their declaration. A "no" is automatically generated if one or both of the EPDs is missing at least one of these elements.

\subsubsection{Scoring}

\begin{tabular}{|c|c|c|c|c|c|}
\hline $\begin{array}{l}\text { CRUCIAL: CAN } \\
\text { BE COMPARED? }\end{array}$ & CRUCIAL NO & CAUTIOUS NO & TOTAL NO & PCR Same & SCORE \\
\hline no & 1 & 8 & 9 & No & c \\
\hline no & 2 & 7 & 9 & No & 0 \\
\hline yes & 0 & 4 & 4 & Yes & 16 \\
\hline no & 1 & 5 & 6 & No & 0 \\
\hline no & 1 & 4 & 5 & Yes & 0 \\
\hline no & 2 & 6 & 8 & No & 0 \\
\hline yes & 0 & 4 & 4 & Yes & 16 \\
\hline no & 1 & 8 & 9 & No & 0 \\
\hline no & 2 & 7 & 9 & No & 0 \\
\hline yes & 0 & 4 & 4 & Yes & 16 \\
\hline yes & 0 & 4 & 4 & Yes & 16 \\
\hline yes & 0 & 4 & 4 & Yes & 16 \\
\hline no & 2 & 7 & 9 & No & 0 \\
\hline no & 2 & 6 & 8 & No & 0 \\
\hline no & 1 & 8 & 9 & No & 0 \\
\hline no & 1 & 7 & 8 & No & 0 \\
\hline no & 1 & 8 & 9 & No & 0 \\
\hline no & 2 & 6 & 8 & No & 0 \\
\hline no & 1 & 8 & 9 & No & 0 \\
\hline yes & 0 & 5 & 5 & No & 15 \\
\hline
\end{tabular}

Table 20: EPD Comparison Matrix Scoring Example

The Scoring Component, shown in Table 21, includes 8 significant columns, 2 of which are not shown in the table.

First, the "Crucial: Can be Compared?" column, is automatically generated based on the results of the Comparison section. A "yes" is generated if all the crucial categories (from Section 4.3.1) are all "yes"s, which essentially means the EPDs can be compared according to ISO 14025 
6.7.2 Requirements for comparability. A "no" is generated if at least one of the crucial categories contains a "no".

The three succeeding columns, titled: "Crucial No", "Cautious No", and "Total No" quantify the results from the Comparison section. The "Crucial No" column quantifies the amount of "no"s from the elements in Section 4.3.1, and the "Cautious No" column quantifies the amount of "no"s from the elements in Section 4.3.2. The "Total No" column is a sum of two prior columns.

The columns titled "PCR Same" has two results: "Yes", if the PCR of the two products being compared are the same, and "No", if the PCRs of the two products being compared are different.

The "Score" column scores the EPDs based on their comparability. The score value starts at 20, and is subtracted from based on the amount of "Crucial No"s and "Cautious No"s the comparison has. A "Crucial No" brings the score to 0, meaning the two EPDs cannot be compared. A "Cautious No" subtracts 1 point from the score.

For a certain comparison, if the "PCR Same" value is "Yes", and the "Score" value is 0, this means there must be a problem with the EPDs adhering to the PCRs. If the "PCR Same" value is "No", and the "Score" value is greater than 0 , this means that comparison can be done even though the two EPDs have different PCRs. These two columns mark where these instances occur, which can be seen in Table 22.

\begin{tabular}{rrr} 
PCR Same & SCORE & \\
20 & 14 & \\
20 & 16 & Permissible comparison, both EPDs use the same PCR \\
\hline 0 & 15 & Permissible comparison, both EPDs use the same PCR \\
20 & 16 & Comparison with different PCRs \\
0 & 0 & Problem with PCR or EPD \\
0 & 0 & Not comparable \\
20 & 0 & Not comparable \\
20 & 0 & Problem with PCR or EPD \\
\hline
\end{tabular}

Table 21: EPD Comparison Matrix Scoring Sample 


\subsection{Impact Category Results}

This section is an expansion of the inputs required for Section 4.1.4, Impact Categories. This table includes columns beside each impact category to input the total impacts for each product's lifecycle. This is shown in Table 23 below.

\section{Impact Categories with Values (ISO 21930 8.2.2)}

\begin{tabular}{|c|c|c|c|c|c|c|c|}
\hline $\begin{array}{c}\text { Global } \\
\text { Warming } \\
\text { Potential }\end{array}$ & $\begin{array}{l}\text { GWP } \\
\text { Total }\end{array}$ & $\begin{array}{c}\text { Ozone } \\
\text { Depletion }\end{array}$ & OD Total & Acidification & A Total & Eutrophication & E Total \\
\hline $\mathrm{kg} \mathrm{CO} \mathrm{CO}_{2}$ eq & $6.60 E+00$ & $\begin{array}{c}\text { kg CFC-11 } \\
\text { eq }\end{array}$ & $0.00 E+00$ & $\mathrm{~kg} \mathrm{SO}_{2}$ eq & 4.55E-02 & $\mathrm{kg} \mathrm{PO}_{4} \mathrm{eq}$ & 7.00E-03 \\
\hline $\mathrm{kg} \mathrm{CO} 2$ eq & $2.84 \mathrm{E}+00$ & $\begin{array}{c}\text { kg CFC-11 } \\
\text { eq }\end{array}$ & 4.05E-08 & $\begin{array}{c}\text { Converted } \\
\text { from mol H+ } \\
\text { eq }\end{array}$ & $5.88 \mathrm{E}-02$ & $\begin{array}{l}\text { Converted } \\
\text { from kg N eq }\end{array}$ & 3.79E-03 \\
\hline $\mathrm{kg} \mathrm{CO}_{2}$ eq & $5.02 E+00$ & $\begin{array}{c}\text { kg CFC-11 } \\
\text { eq }\end{array}$ & $3.52 \mathrm{E}-09$ & $\mathrm{~kg} \mathrm{SO}_{2}$ eq & $1.90 \mathrm{E}-02$ & $\mathrm{~kg} \mathrm{PO}_{4}$ eq & $2.12 \mathrm{E}-03$ \\
\hline $\mathrm{kg} \mathrm{CO} 2 \mathrm{eq}$ & $5.52 E+01$ & $\begin{array}{c}\text { kg CFC-11 } \\
\text { eq }\end{array}$ & $3.44 \mathrm{E}-09$ & $\mathrm{~kg} \mathrm{SO}_{2}$ eq & 1.82E-01 & $\mathrm{kg} \mathrm{PO}_{4}$ eq & 1.27E-02 \\
\hline
\end{tabular}

Table 22: EPD Comparison Matrix Impact Category Results Sample

Ideally, this section would be used after two EPDs have been deemed comparable by the methodology outlined in Sections 4.1, 4.2, and 4.3. This section deepens this method by allowing a side-by-side comparison of the environmental impact data within each comparable EPD. 


\section{Design of the Product Category Rule Comparison Matrix}

The PCR Comparison Matrix presented in this section was conceived using a previously developed PCR Comparison Template by Subramanian, Ingwersen and Hensler (2011) as its foundation. The PCR Comparison Template uses criteria within a PCR and enables a side-byside comparison of two PCRs based on the information input in the chart. An excerpt of this chart is shown in Table 24.

\begin{tabular}{|c|c|c|c|c|}
\hline CRITERIA & DESCRIPTION & PCR\#1 & PCR \#2 & COMMENTS \\
\hline \multicolumn{5}{|l|}{$\begin{array}{l}\text { General } \\
\text { Information }\end{array}$} \\
\hline Product & $\begin{array}{l}\text { Provide the name of the } \\
\text { product category that this } \\
\text { PCR is targeted at }\end{array}$ & & & \\
\hline $\begin{array}{l}\text { CPC Module (if } \\
\text { applicable) }\end{array}$ & $\begin{array}{l}\text { Provide the entire hierarchy } \\
\text { (names and codes) that } \\
\text { leads up to the product } \\
\text { category and its categories if } \\
\text { applicable }\end{array}$ & & & \\
\hline $\begin{array}{l}\text { UNSPSC Module } \\
\text { (if applicable) }\end{array}$ & $\begin{array}{l}\text { Provide the entire hierarchy } \\
\text { (names and codes) that } \\
\text { leads up to the product } \\
\text { category and its categories if } \\
\text { applicable }\end{array}$ & & & \\
\hline $\begin{array}{l}\text { GPC Module (if } \\
\text { applicable) }\end{array}$ & $\begin{array}{l}\text { Provide the entire hierarchy } \\
\text { (names and codes) that } \\
\text { leads up to the product } \\
\text { category and its categories if } \\
\text { applicable }\end{array}$ & & & \\
\hline
\end{tabular}

Table 23: PCR Comparison Template based on (Subramanian, Ingwersen \& Hensler, 2011)

In this PCR Comparison Template, there is no ability to compare multiple PCRs in the same matrix, meaning you would have to complete multiple templates in order to compare more than two PCRs. Additionally, there are no provisions for establishing which of these criteria regarding comparability either do not matter, should be analyzed with some level of caution (equivalent in the ISO standard), or are crucial for comparison (identical in the ISO standard). This chart uses the same general idea as the EPD Comparison Matrix explained in Section 5, which determines what comparisons can be made and provides scores for each comparison, and adapts it for the comparison of PCRs.

Figure 5 illustrates an overview of the PCR Comparison Matrix. Each of the chart's separate sections and functions is explained in this section. 


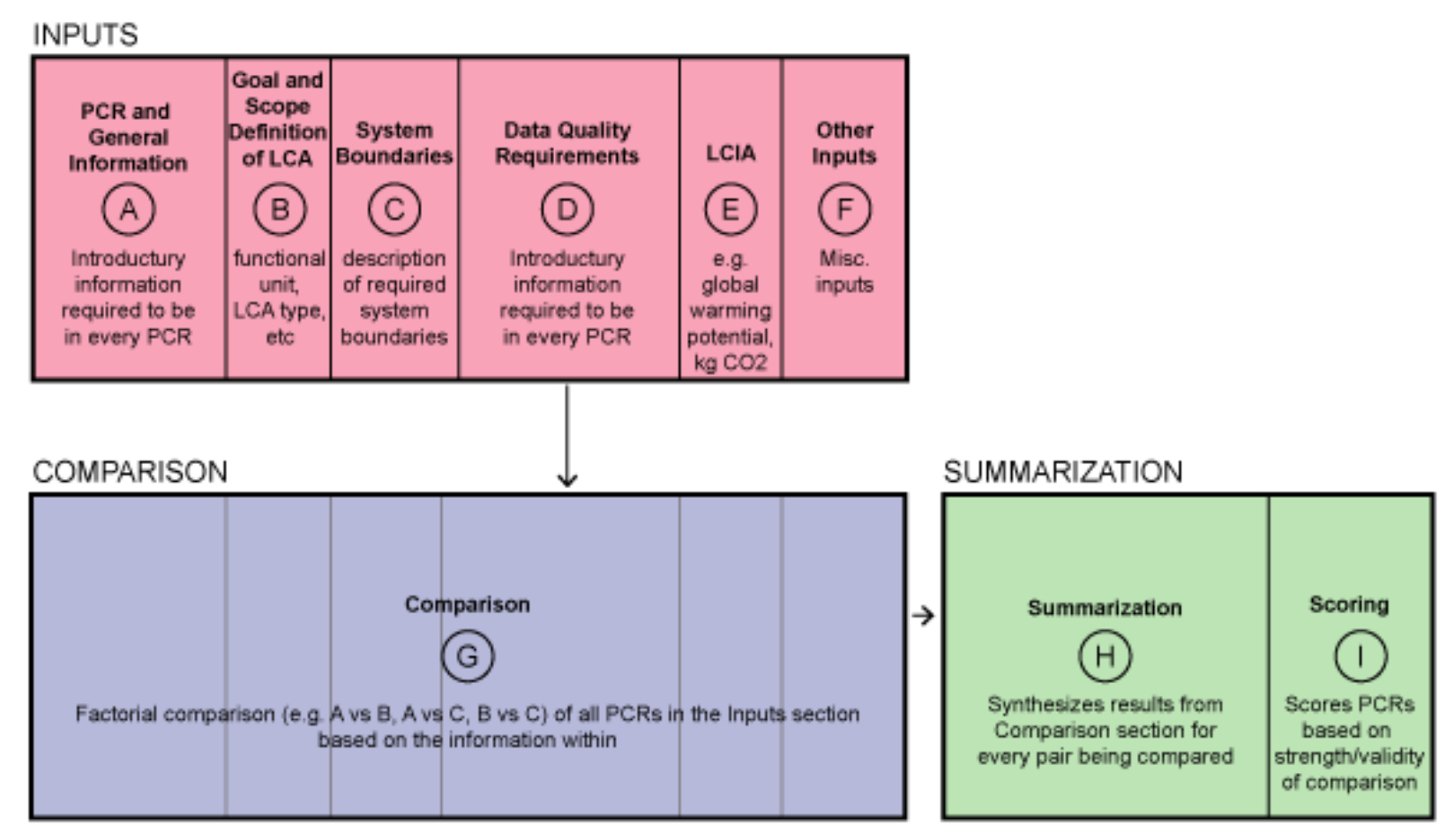

Figure 5: PCR Comparison Matrix Overview

The breakdown for discussion on building the PCR Comparison Matrix is very similar to Section 4, regarding the EPD Comparison Matrix. The following sections discuss each component.

Section 5.1 discusses the inputs (A-I in Figure 5). Part A contains General Information, which includes basic information regarding the PCR and the governing standards; this is discussed in Section 5.1.1. Part B covers the Goal and Scope Definition of the LCA; this is discussed in Section 5.1.2. Part $C$ outlines the System Boundaries mentioned in the $P C R$, and is elaborated on in Section 5.1.3. Part $D$ focuses on data quality requirements, along with criteria for primary/foreground and secondary/background data selection, conglomerated into a section titled Data Provisions discussed in Section 5.1.4. Part E, focuses on LCIA inputs, including the impact categories and characterization factors required to be in every resulting EPD. Part $F$ encompasses all other inputs, which includes a Communication section (format and structure requirements), the system of measurement mandated in the PCR, required additional environmental information, and requirements for hazardous substances. These factors are discussed in Section 5.1.5.

Part G (described in Section 5.2) describes the Comparison process that uses the information within the Inputs section to make detailed comparisons for every critical component of the PCR 
Comparison Matrix. Each section that is included in Section 5.1 is reflected in Section 5.2 as subsections, where the comparison process is described in detail.

Parts $\mathrm{H}$ and I (described in Section 5.3) focus on the Summarization stage of the matrix, which uses the detailed Comparison section to provide overviews for items within the matrix whose inclusions are crucial, or should be compared with caution. Part $\mathrm{G}$ focuses on the summarization aspect of this section and is described in Section 5.3.1, while Part $\mathrm{H}$ discusses the scoring process for each comparison in the matrix and is elaborated on in Section 5.3.2.

\subsection{Inputs}

As the PCR Comparison Matrix was built after the EPD Comparison Matrix, the workflow and framework to enable comparisons was established. Similar to Section 4.1, the specific contents of the PCRs that are necessary for comparison needed to be identified, and it needed to be determined whether the content should be: a) compared with caution, or b) crucial for comparison. Cues were taken from the standards outlined in Section 2.5, which include ISO 14025, ISO 14044, ISO 21930, and EN 15804. Also, the Guidance for Product Category Rule Development (2013) was used to help build the PCR Comparison Matrix. While not an international standard, the guidance document is part of an international initiative by EPD experts and organizations to build on existing best practices and provide internationally relevant guidance to the development of PCRs.

The majority of the content for the matrix is taken from ISO 14025, but other standards and guidance documents added other metrics for comparison where appropriate for construction products. Additionally, some comparison metrics that were not included in any of these standards, but were judged as important characteristics for the comparability of EPDs were included in the matrix.

Exactly like the EPD Comparison Matrix, ISO 14025 6.7.2 Requirements for comparability, states that Type III environmental declarations shall be deemed to have achieved comparability when a list of conditions has been met. This list includes conditions that are required to be "equivalent", and conditions required to be "identical". This is also reflected in the Guidance for Product Category Rule Development, in clearer and more explicit terms in ISO 14025 Section 6.2 Comparability of Claims.

This was directly implemented in the chart to inform whether a section should be compared with caution, or crucial for comparison. One major deficiency in ISO 14025 is that equivalency is not 
defined, and therefore it is up to the user to decide whether the information is equivalent or not. For the purposes of this comparison, equivalence is defined as statements with the same scope and fulfill the same purpose or goal.

Similar to the guidelines established in 4.1, crucial elements are those that if not present, not adequately defined, or accounted for differently preclude the comparison of PCRs. Elements that require caution are those that have the potential for errors to be introduced into the comparison. The content, language, and terms used in the matrix were taken from the above set of international standards.

\subsubsection{General Information}

\begin{tabular}{|c|c|c|c|c|c|c|c|}
\hline & & & & NERAL IN & ATION & IDENTICAL & \\
\hline \# & $\begin{array}{l}\text { Programme } \\
\text { Operator }\end{array}$ & PCR & ISO 14025 & EN 15804 & PCR ID & Product & CPC Module \\
\hline A & $\begin{array}{l}\text { International } \\
\text { EPD System }\end{array}$ & $\begin{array}{l}2012.01 \\
\text { Version } 1.2 \\
\text { for } \\
\text { Construction } \\
\text { Products }\end{array}$ & & & 2012:01 & $\begin{array}{l}\text { "construction } \\
\text { products and } \\
\text { construction } \\
\text { services" }\end{array}$ & $\begin{array}{l}\text { Long list of } \\
\text { CPC codes, } \\
\text { available in } \\
\text { PCR } \\
\text { document. }\end{array}$ \\
\hline B & $\begin{array}{l}\text { UL } \\
\text { Environment }\end{array}$ & $\begin{array}{l}\text { Building } \\
\text { Envelope } \\
\text { Thermal } \\
\text { Insulation } \\
\text { v1.2 }\end{array}$ & & & 000001 & $\begin{array}{l}\text { "building } \\
\text { envelope } \\
\text { thermal } \\
\text { insulation" }\end{array}$ & N/A \\
\hline
\end{tabular}

Table 24: Sample entries from PCR Comparison Matrix, General Information section

The General Information section establishes introductory information for the rest of the PCR Comparison Matrix. Table 25 above only shows a small portion of the section. The General Information section includes:

- Name of the program operator and PCR;

- Governing standards: if the PCR is written under ISO 14025 and/or EN 15804;

- PCR ID;

- Product: the name or description of the category the PCR is targeted at, word-for-word from PCR;

- CPC, UNSPSC, GPC Modules: Central Product Classification, United Nations Standard Products and Services Code, and Global Product Classification, a series of product 
classification systems. This section asks for their inclusion in the comparison chart if they are included in the PCR;

- Language of PCR;

- Date of Publication, and Date of Expiration;

- Schedule for Renewal of PCR;

- Geographic Region the PCR serves, and any local/regional provisions;

- External Critical Review: was the PCR reviewed by an external body as part of the publishing process?;

- Reasoning for the Development of Superseding PCR: if an old PCR exists, and this is included in the PCR's text, and;

- Version of ISO 14025 used: what version of ISO 14025 was used to develop the PCR.

Only the Product column is required to be equivalent for comparison in this section because it is the only column which information which could potentially have an impact on the comparison (i.e. two PCRs written for dissimilar products cannot be compared). 


\subsubsection{Goal and Scope Definition of LCA}

Table 24 shows two PCRs compared in the Goal and Scope Definition of LCA section.

The Goal and Scope Definition of LCA includes information that is important to the background of the LCA and how it should be read. The only column in this section that is required to be identical or equivalent is the functional unit column. The functional unit must be identical, according to ISO 14025 6.7.2 Requirements for comparability.

\begin{tabular}{|c|c|c|c|c|c|}
\hline \multicolumn{6}{|c|}{ GOAL AND SCOPE DEFINITION } \\
\hline $\begin{array}{l}\text { Provide the } \\
\text { functional } \\
\text { unit of the } \\
\text { product as } \\
\text { described in } \\
\text { the PCR }\end{array}$ & $\begin{array}{l}\text { Attributional or } \\
\text { consequential }\end{array}$ & $\begin{array}{l}\text { Provide the intended } \\
\text { application of the PCR } \\
\text { as described in the } \\
\text { PCR }\end{array}$ & $\begin{array}{c}\text { Provide the } \\
\text { intended } \\
\text { audience of the } \\
\text { PCR as described } \\
\text { in the PCR }\end{array}$ & $\begin{array}{l}\text { Provide the } \\
\text { function of } \\
\text { the product } \\
\text { as described } \\
\text { in the PCR }\end{array}$ & $\begin{array}{c}\text { Included? } \\
\text { Yes/No }\end{array}$ \\
\hline $\begin{array}{l}\text { Functional } \\
\text { Unit }\end{array}$ & LCA Type & Intended Application & $\begin{array}{l}\text { Intended } \\
\text { Audience }\end{array}$ & Function & $\begin{array}{l}\text { Process } \\
\text { Flow } \\
\text { Diagram }\end{array}$ \\
\hline $\begin{array}{l}\text { N/A, only } \\
\text { includes } \\
\text { declared } \\
\text { units: } \\
\text { Mass, } \\
\text { volume, area, } \\
\text { length, or an } \\
\text { item }\end{array}$ & Attributional & $\begin{array}{l}\text { Applicable as a PCR } \\
\text { directly, or a "PCR } \\
\text { Basic Module", i.e. } \\
\text { the template/starting } \\
\text { point for } \\
\text { development of more } \\
\text { detailed PCRs. }\end{array}$ & $\begin{array}{l}\text { For } \\
\text { manufacturers } \\
\text { to develop EPDs, } \\
\text { or for the } \\
\text { development of } \\
\text { more specific } \\
\text { PCRs }\end{array}$ & $\begin{array}{l}\text { All } \\
\text { construction } \\
\text { products and } \\
\text { construction } \\
\text { services for } \\
\text { buildings }\end{array}$ & YES \\
\hline $\begin{array}{l}1 \mathrm{~m}^{2} \text { of } \\
\text { insulation } \\
\text { material with } \\
\text { thickness that } \\
\text { gives a design } \\
\text { thermal } \\
\text { resistance of } \\
\mathrm{R}=1 \mathrm{~m}^{2} \mathrm{~K} / \mathrm{W}\end{array}$ & Attributional & $\begin{array}{l}\text { For all building } \\
\text { envelope thermal } \\
\text { insulation and } \\
\text { mechanical insulation } \\
\text { materials }\end{array}$ & $\begin{array}{l}\text { Manufacturers } \\
\text { of building } \\
\text { envelope } \\
\text { thermal } \\
\text { insulation and } \\
\text { mechanical } \\
\text { insulation } \\
\text { materials }\end{array}$ & $\begin{array}{l}\text { Insulation for } \\
\text { building } \\
\text { envelope or } \\
\text { mechanical } \\
\text { systems }\end{array}$ & NO \\
\hline
\end{tabular}

Table 25: Sample entries from PCR Comparison Matrix, Goal and Scope Definition of LCA section

This differs from the requirements for the functional/declared unit in the EPD Comparison Matrix, which requires the functional unit to be equivalent, meaning that it can be converted or scaled to match. However, the end-uses of the PCR and the EPD vary. Some PCRs, as can be seen in Table 26, mandate that the declared unit can be anything, as long as it falls into a category: a mass, a volume, an area, a length, or an item. Therefore, two EPDs following the same PCR can have wildly different declared units. Because of this, the requirement for the PCR Comparison Matrix is the functional unit must be identical in type, but the quantity may vary. 


\subsubsection{System Boundaries}

The System Boundaries section of the PCR Comparison Matrix asks users to input all possible elements of the system boundaries according to the PCR, separated into 5 categories: Raw Material Acquisition Stage, Production Stage, Distribution Stage, Use Stage, and End of Life Stage. System boundaries between two PCRs are required to be identical for comparison.

The information within the system boundaries section of the PCR does not necessarily need to be included in an EPD that is written according to the PCR. The inclusion of such system boundaries is optional in this instance; the PCR acts more like a "best practice guideline" rather than a mandatory checklist.

Table 27 (next page) shows a sample of the System Boundaries section in the PCR Comparison Matrix. 


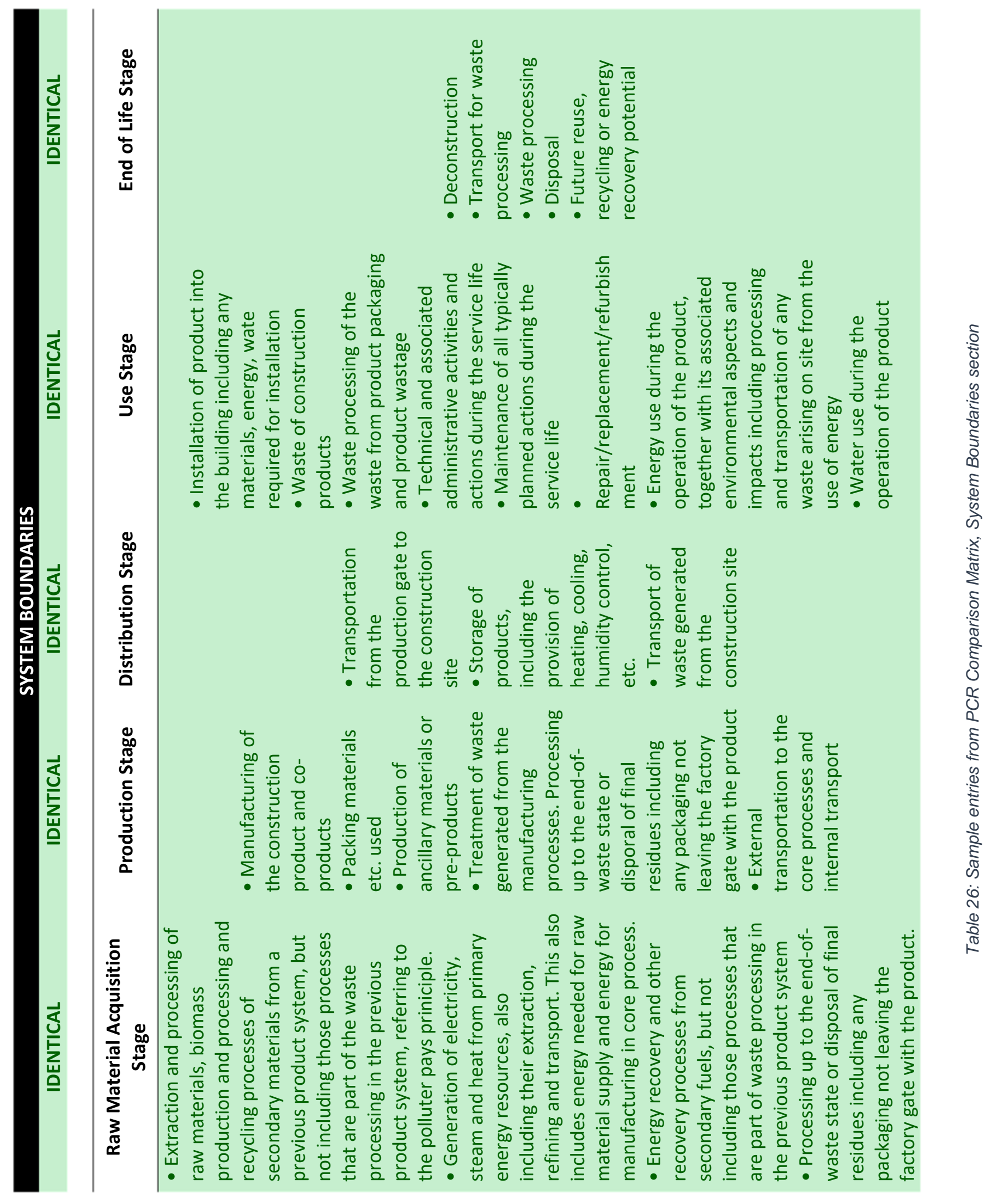




\subsubsection{Data Provisions}

This Data Provisions section will be split into two parts; the first discussing data quality requirements, and the second discussing primary and secondary data requirements.

\subsubsection{Data Quality Requirements}

\begin{tabular}{|c|c|c|c|c|}
\hline \multicolumn{5}{|c|}{ GENERAL DATA/DATA QUALITY REQUIREMENTS } \\
\hline \multicolumn{3}{|c|}{ IDENTICAL } & IDENTICAL & IDENTICAL \\
\hline \multicolumn{3}{|c|}{$\begin{array}{l}\text { coverage, precision, completeness, } \\
\text { representativeness, consistency, } \\
\text { reproducibility, sources and uncertainty }\end{array}$} & $\begin{array}{l}\text { Provide any general cut- } \\
\text { off criteria that is to be } \\
\text { applied all processes }\end{array}$ & $\begin{array}{c}\text { Provide any general allocation } \\
\text { methods that is to be applied when } \\
\text { need arises }\end{array}$ \\
\hline $\begin{array}{c}\text { Temporal } \\
\text { Requirements }\end{array}$ & $\begin{array}{l}\text { Technological } \\
\text { Requirements }\end{array}$ & $\begin{array}{l}\text { Geographic } \\
\text { Requirements }\end{array}$ & $\begin{array}{l}\text { Cut-off Rules (Criteria for } \\
\text { the Exclusion of Inputs and } \\
\text { Outputs/Flows) }\end{array}$ & $\begin{array}{c}\text { Allocation Rules (of Material and Energy } \\
\text { Flows and Releases) }\end{array}$ \\
\hline $\begin{array}{l}\text { Data sets used } \\
\text { for calculation } \\
\text { shall have } \\
\text { been updates } \\
\text { within the last } \\
10 \text { years for } \\
\text { generic data, } \\
\text { last } 5 \text { years for } \\
\text { specific data. } \\
\text { Data sets shall } \\
\text { be based on } 1 \\
\text { year averaged } \\
\text { data. }\end{array}$ & Not included. & $\begin{array}{l}\text { The data for the } \\
\text { core module } \\
\text { shall be } \\
\text { representative } \\
\text { for the actual } \\
\text { production } \\
\text { processes and } \\
\text { representative } \\
\text { for the } \\
\text { site/region } \\
\text { where the } \\
\text { respective } \\
\text { process is } \\
\text { taking place }\end{array}$ & $\begin{array}{l}\text { Minimum of } 95 \% \text { of total } \\
\text { inflows to the upstream and } \\
\text { core module shall be } \\
\text { included. }\end{array}$ & $\begin{array}{l}\text { - the initial allocation step includes } \\
\text { dividing up the system sub-processes and } \\
\text { collecting the input and output data } \\
\text { related to these sub-processes. } \\
\text { - the first (preferably) allocation } \\
\text { procedure step for each sub-process is to } \\
\text { partition the inputs and outputs of the } \\
\text { system in to their different products in a } \\
\text { way that reflects the underlying physical } \\
\text { relationships between them. } \\
\text { - the second (worst case) allocation } \\
\text { procedure step is needed when physical } \\
\text { relationship alone cannot be established } \\
\text { or used as the basis for allocation. In this } \\
\text { case, the remaining environmental inputs } \\
\text { and outputs from a sub-process must be } \\
\text { allocated between the products in a way } \\
\text { that reflects other relationships between } \\
\text { them, such as the economic value of the } \\
\text { products. }\end{array}$ \\
\hline $\begin{array}{l}\text { - Specific data } \\
\text { no older than } \\
3 \text { years. } \\
\text { Generic data } \\
\text { no older than } \\
10 .\end{array}$ & $\begin{array}{l}\text { Data will } \\
\text { represent } \\
\text { technology in } \\
\text { use. }\end{array}$ & $\begin{array}{l}\text { The geographic } \\
\text { region of the } \\
\text { product sites } \\
\text { included in the } \\
\text { calculation of } \\
\text { representative } \\
\text { data will be } \\
\text { documented. }\end{array}$ & $\begin{array}{l}\text { A process or activity that } \\
\text { contributes no more than } \\
2 \% \text { of the total mass and } 1 \% \\
\text { of the total energy use may } \\
\text { be omitted from the } \\
\text { inventory analysis. } \\
\text { Omissions of any materials } \\
\text { flows that may have a } \\
\text { relevant contribution to the } \\
\text { selected impact categories } \\
\text { of the products underlying } \\
\text { the EPD will be justified, if } \\
\text { applicable, by a sensitivity } \\
\text { analysis. }\end{array}$ & $\begin{array}{l}\text { For production of building envelope } \\
\text { thermal insulation products, the preferred } \\
\text { allocation rule is that allocation be carried } \\
\text { out according to mass. For additional } \\
\text { information, see ISO } 14044 \text { Section } 4.3 .4 \text {. }\end{array}$ \\
\hline & & & $\begin{array}{l}\text { The sum of the excluded } \\
\text { material flows must not } \\
\text { exceed } 5 \% \text { of mass, energy, } \\
\text { or environmental relevance. }\end{array}$ & \\
\hline
\end{tabular}

Table 27: Sample entries from PCR Comparison Matrix, Data Provisions I section 
The first portion of the Data Provisions section, which focuses on data quality requirements, is illustrated in Table 28. The first three columns ask for the temporal, technological, and geographical requirements for the data sets used in the LCA. The fourth and fifth columns ask for the cut-off and allocation rules to be applied during the LCA process. Each cell used the full quotation from the PCR, although a user could simplify this if desired. All of these elements within this portion are required to be identical between two PCRs for comparison, as per ISO 14025 6.7.2 Requirements for comparability.

\subsubsection{2. $\quad$ Primary and Secondary Data}

The second portion of the Data Provisions section requires inputs regarding primary (foreground) and secondary (background) data requirements, illustrated in Table 29. The first column's inputs are the method of primary data collection each PCR outlines. This can include data sheets, software tools, or suggestion means for data collection for the primary LCA data. The five succeeding columns ask for the names of processed for which primary data must be collected/used, in the same separation as used in Section 5.1.3: Raw Material Acquisition Stage, Production Stage, Distribution Stage, Use Stage, and End of Life Stage. If the PCR does not specify a process for any of the stages, "None" or a similar phrase should be input. The last column, which addresses secondary (background) data, asks for the data sources - or the criteria that can be used to select a data source - for the secondary data in the LCA. Each of these columns are required to be equivalent, as per ISO 14025 6.7.2 Requirements for comparability. 


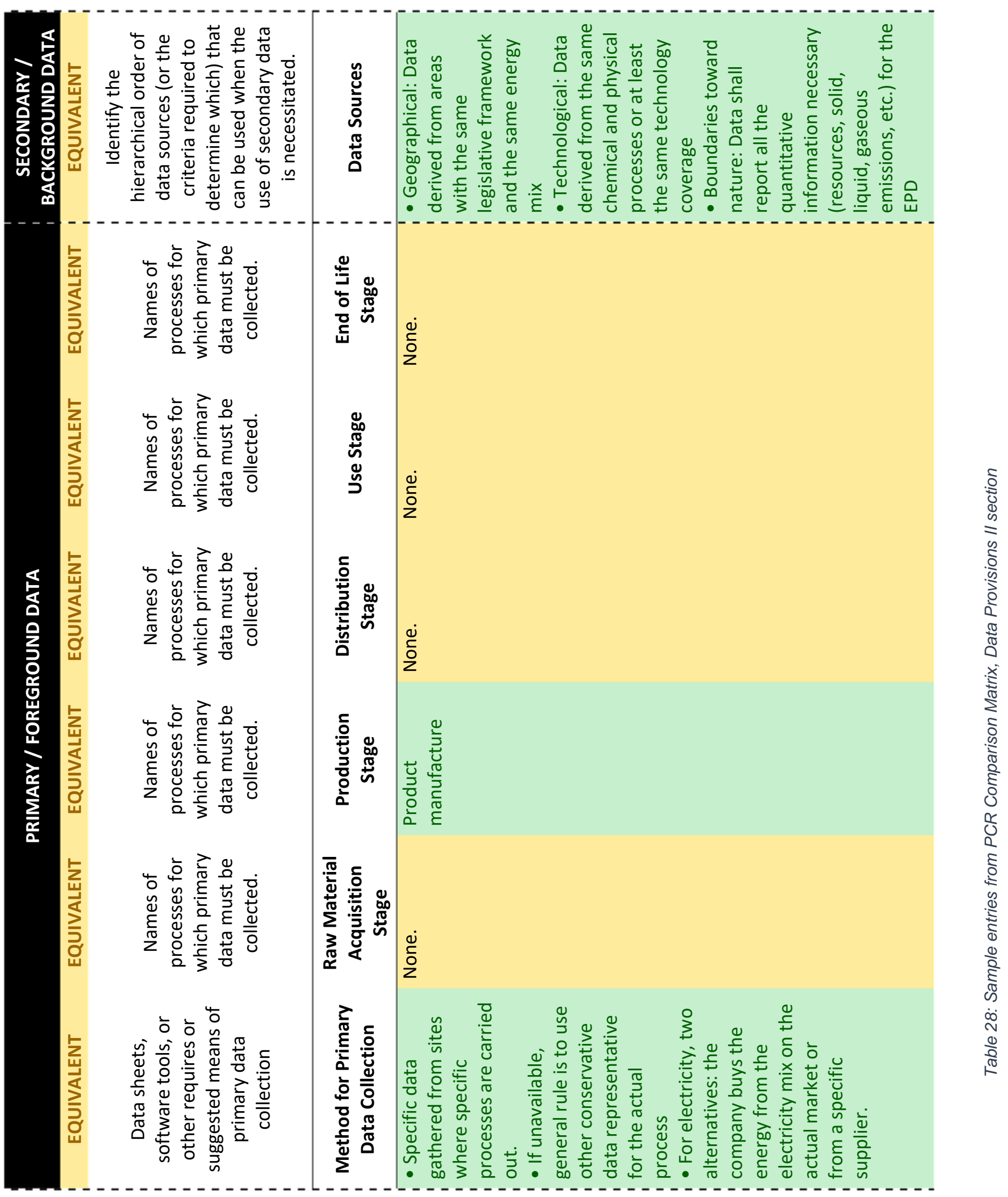




\subsubsection{Life Cycle Impact Assessment}

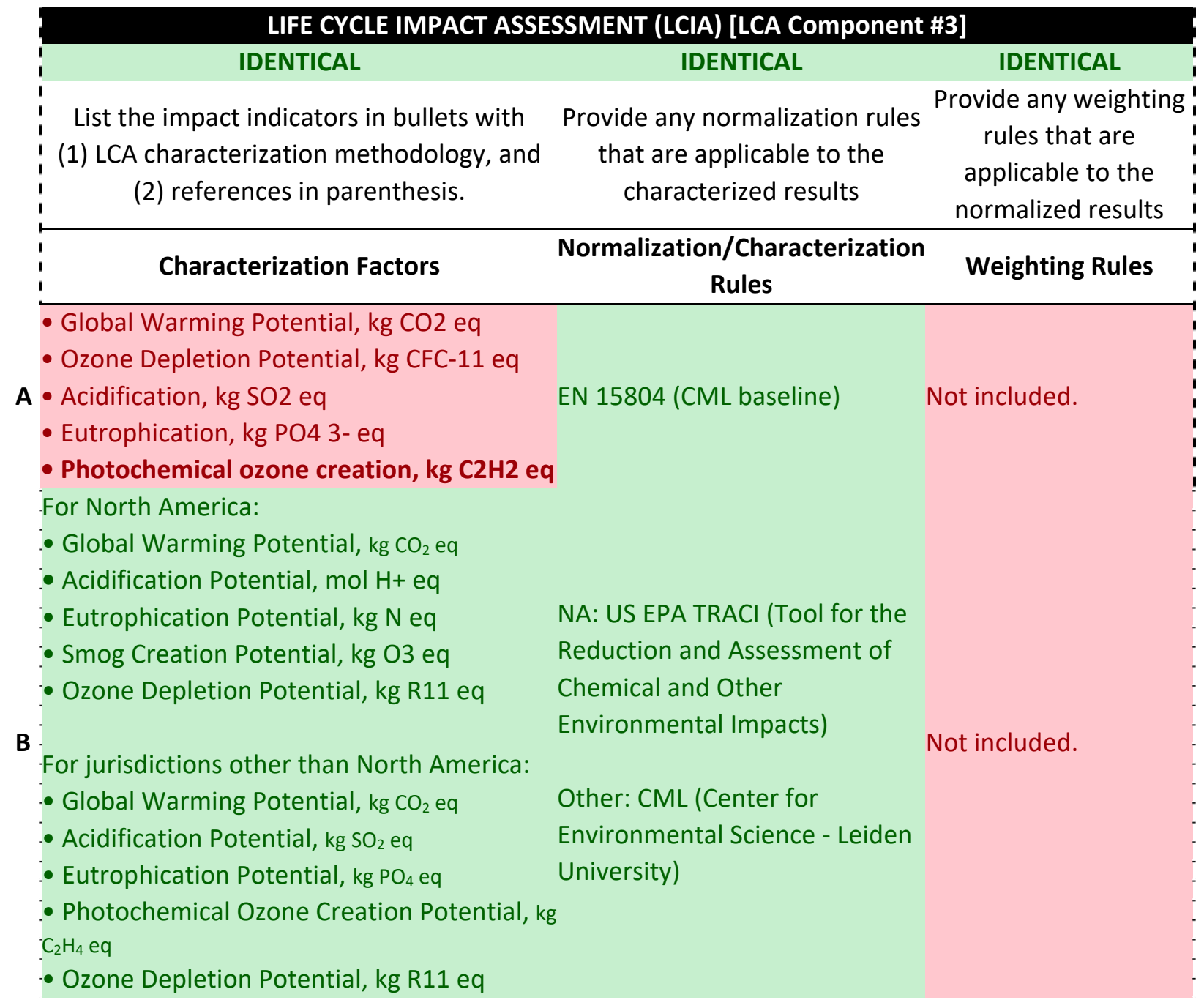

Table 29: Sample entries from PCR Comparison Matrix, Life Cycle Impact Assessment section

This section focuses on components specific to the Life Cycle Impact Assessment portion of the LCA. The first column, characterization factors, are the environmental impact categories, and the indicators for these (the "units") that are to be used in the EPD. The definition of these characterization factors are described in Section 4.1.4 of this thesis. The last two columns include Normalization/Characterization Rules, which can be defined as "calculating the magnitude of the category indicator results", applied to particular substances in a product, and Weighting Rules, "assignment and calculation of different impact categories and resources reflecting the relative importance" (DMotE, 2006). Each normalization scheme has different provisions for normalization and weighting of environmental indicator results. These two columns require the respective normalization and weighting rules used for LCIA. All columns are 
required to be identical for two PCRs to be comparable, as per ISO 14025 6.7.2 Requirements for comparability.

In Table 30 above, EPD A and EPD B are incomparable based on the characterization factors column. EPD A states photochemical ozone creation in stated in $\mathrm{kg} \mathrm{C}_{2} \mathrm{H}_{2}$ eq (ethyne), rather than $\mathrm{kg} \mathrm{C}_{2} \mathrm{H}_{4}$ eq (ethene), which was the most common characterization factor for that impact category.

\subsubsection{Other Inputs}

This section includes columns that do not fit in any other section in the PCR Comparison Matrix. These include:

- Format and Structure: the required format for presenting environmental impact information in the EPD, and the structure of the document;

- Units: the system of measurement to be used in the EPD;

- Additional Environmental Information: any additional environmental information that is to be declared in the EPD (often related to indoor health or regional standards/laws);

- Hazardous Substances: a list of hazardous substances that are required to be declared in the EPD if used as part of the manufacturing process

- Safety Information: safety information requirements regarding handling, use, and installation;

- Glossary: identifying if a glossary is included in the PCR;

- Reference Literature: identifying if any reference literature is used in the PCR, including standards and past PCR documents;

- General Comments: a cell for any information within the PCR that does not fit into any of the preceding categories in the PCR Comparison Matrix,

- File Path: the local file path of the PCR, and;

- Notes: general notes on the PCR.

The Additional Environmental Information and Hazardous Substances columns are required to be equivalent. Units are required to be identical as per ISO 14025 6.7.2 Requirements for comparability, but the reasoning for their exclusion has been elaborated on in Section 4.1.3.5 of this thesis. There are no other requirements for the other columns. This section is shown in Table 31. 


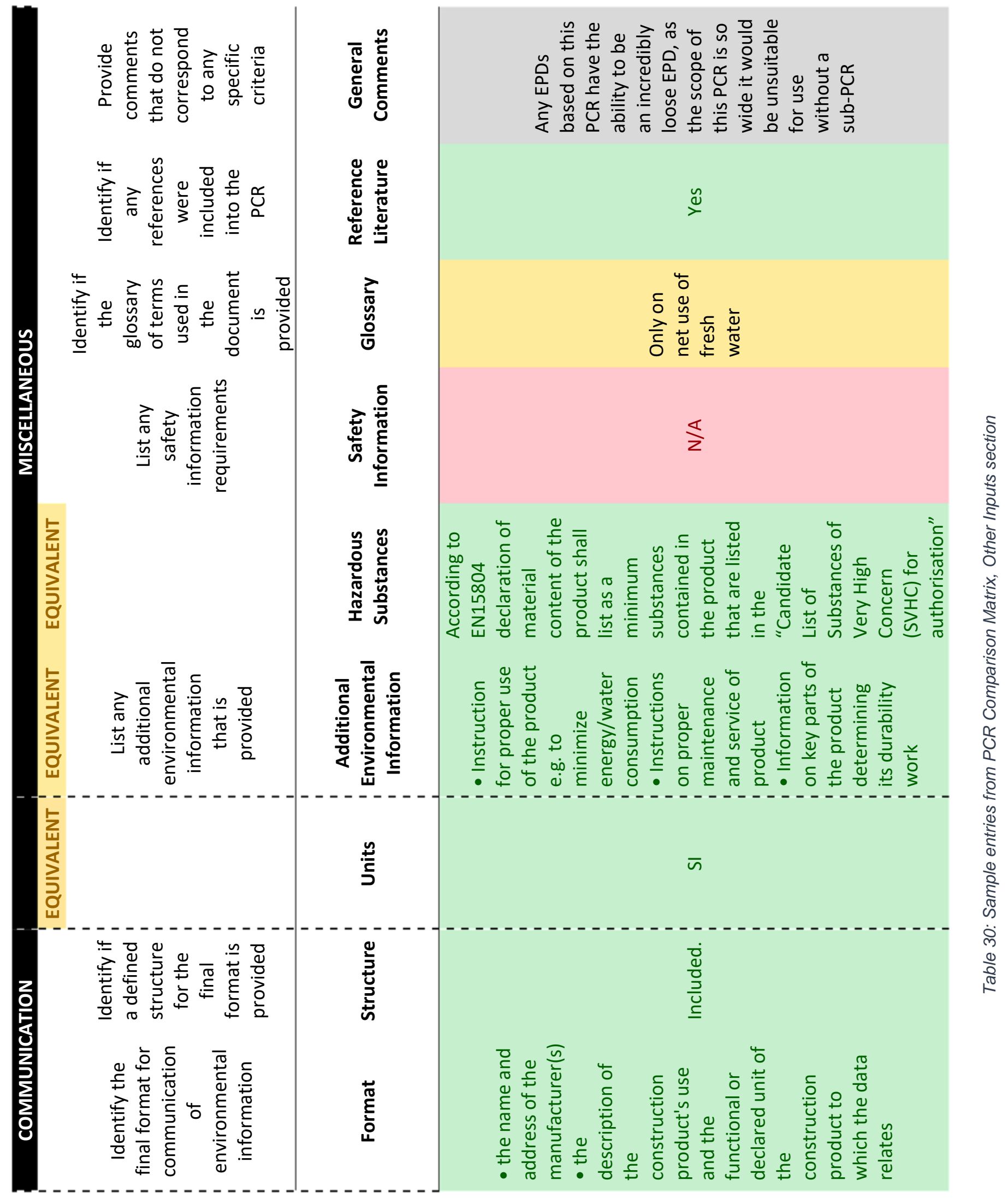




\subsection{Product Category Rule Comparison}

This section of the chart, along with the previous, is formatted in a similar manner to that in Sections 4.1 and 4.2 .

This section of the chart uses the information from the Inputs section for factorial comparisons. Every possible comparison within the matrix is included in this section in a factorial fashion. Every EPD is assigned a letter (A, B, C...) and is then compared to every other PCR in the chart (A vs $B, A$ vs $C, B$ vs $C \ldots$..).

The comparison is populated with either a "yes" or "no", depending on the section. A "yes" constitutes that the EPD will be required to disclose information in the same way, and a "no" means one or both do not require disclosure in either the same or an appropriate way. However, this definition alters depending on which section from the Inputs is being analyzed. An example of the Comparison section is shown in Table 32.

\begin{tabular}{lcc}
\hline Comparison & $\begin{array}{c}\text { IDENTICAL } \\
\text { Column (References sections } \\
\text { described previously) }\end{array}$ & $\begin{array}{c}\text { EQUIVALENT } \\
\text { Column (References sections } \\
\text { described previously) }\end{array}$ \\
COMPARABILITY & & \\
\hline A vs B & yes & no \\
A vs C & yes & yes \\
A vs D & yes & yes \\
A vs E & yes & yes \\
B vs C & yes & no \\
B vs D & yes & no \\
B vs E & yes & no
\end{tabular}

Table 31: Sample entries from PCR Comparison Matrix Comparison Section

This Comparison section is organized in the same fashion as discussed in Section 6.1 Inputs, as it is a reflection of that part of the matrix.

\subsubsection{General Information}

The following columns are compared within the General Information section: adherence to ISO 14025 , adherence to EN 15804, the Product column (the name of the product category the PO has established for the PCR), and the language of the PCR. A "yes" is used in each of these columns if: both PCRs adhere to ISO 14025, both PCRs adhere to EN 15804, both PCRs are written for the same product category, and both PCRs are written in the same language. A "no" 
is used if any of the above statements are untrue for each column. Columns that are required to be identical are the adherence to ISO 14025, the Product column, and the Language of PCR column. The EN 15804 column is required to be equivalent.

\subsubsection{Goal and Scope Definition of LCA}

Two columns are compared in the Goal and Scope Definition of LCA section: the functional/declared unit, and the LCA type. For the first column, a "yes" is used if both PCRs require the use of the same functional/declared unit, or can be easily converted to match. A "no" is used if they both use different functional/declared units and they cannot be converted. This column is required to be identical for comparison.

For the LCA type, a "yes" is used if both PCRs require the use of an attributional LCA, or a consequential LCA. A "no" is used if one requires an attributional LCA, while the other requires a consequential LCA. This column is required to be equivalent for comparison.

\subsubsection{System Boundaries}

All columns in the System Boundaries section are included for comparison. A "yes" is used if the system boundaries in both PCRs being compared are exactly the same, for each stage that is being compared: Raw Material Acquisition Stage, Production Stage, Distribution Stage, Use Stage, and End of Life Stage. A "no" is used if the two PCRs being compared prescribe different system boundaries. All columns must be identical for comparison.

\subsubsection{Data Provisions}

\subsubsection{Data Quality Requirements}

All columns in the Data Provisions section are included for comparison. For the Temporal, Technological, and Geographic Requirements columns, a "yes" is used if both PCRs being compared have the same data requirements in each of the three categories, separately. A "no" is used if the PCRs being compared have differing data requirements.

For the Cut-off Rules (also known as the Criteria for the Exclusion of Inputs and Outputs), a "yes" is used if both PCRs being compared mandate the same cut-off rules for mass, energy use, environmental relevance, and overall total flows. A "no" is used if the cut-off rules differ in the two PCRs being compared.

For the allocation rules, a "yes" is used if the two PCRs being compared use the exact same allocation rules, and a "no" is used if the allocation rules differ. 
All columns in the Data Quality Requirements section must be identical for comparison.

\subsubsection{2. $\quad$ Primary and Secondary Data}

All columns in the Primary Data section are included for comparison. The column titled "Method for Primary Data Collection" uses a "yes" if the two PCRs being compared use the same method for primary data collection, and uses a "no" if they use different methods. For the life cycle stage columns, including the Raw Material Acquisition, Production, Distribution, Use, and End of Life Stages, a "yes" is used if both PCRs being compared mandate primary (specific) data be collected for the same processes (i.e. if two PCRs specify that primary data must be collected for the product manufacture). A "no" is used in two cases: one, if the two PCRs do not specify primary data for the same processes, and two, if one or both of the PCRs specify secondary data.

For the Data Sources column in the Secondary data section, a "yes" is used if the data sources, or the criteria required to determine which can be used, are the same in both PCRs being compared. A "no" is used if they differ in the two PCRs being compared.

All columns within the Primary and Secondary Data section must be equivalent for comparison.

\subsubsection{Life Cycle Impact Assessment}

All columns are compared within the Life Cycle Impact Assessment section.

The Characterization Factors column uses a "yes" if both PCRs being compared use the same characterization factors, both in the impact and the indicator (impact being Global Warming Potential, the indicator being $\mathrm{kg} \mathrm{CO}_{2} \mathrm{eq}$ ), all impacts. A "no" is used if the two PCRs being compared differ in this regard.

The Normalization/Characterization Rules, and the Weighting Rules columns use a "yes" if both EPDs being compared use the same normalization and weighting rules, separately. A "no" is used if both EPDs use different normalization or weighting rules.

All columns within the Life Cycle Impact Assessment section must be identical for comparison.

\subsubsection{Other Inputs}

Three columns are used for comparison in the Other Inputs section, including the Units (or system of measurement), Additional Environmental Information, and Hazardous Substances. 
For the Units column, a "yes" is used if both PCRs that are being compared use the same system of measurement (i.e. both IP or both SI). A "no" is used if both EPDs being compared use different systems of measurement.

The Additional Environmental Information, as well as the Hazardous Substances column, uses a "yes" when both PCRs that are being compared state the same information for each of the columns, separately. A "no" is used when the description for Additional Environmental Information or Hazardous Substances differ between PCRs being compared.

All columns must be equivalent for comparison.

\subsection{Product Category Rule Summarization}

This section will describe the Summarization process that is generated from the completion of the Comparison section in the PCR Comparison Matrix. This process either reflects the cells in the Comparisons section, or conglomerates inputs across columns to provide clearer results. Comparisons between PCRs are then scored based on the results in this section.

The columns will be discussed in this Summarization section, which includes both those in the "Crucial" category (discussed further in Section 5.3.1) and those in the "Cautious" category (discussed further in Section 5.3.2), using these terms as previously defined in Section 4. All columns, and the rationale for why each element has been assigned to the crucial or cautious category, can be seen in Table 33 below. 


\begin{tabular}{|c|c|}
\hline 1) & Cautious Category (refer to 6.3.2) \\
\hline $\begin{array}{l}\text { Both PCRs are written for the same } \\
\text { product category, in definition and } \\
\text { description - PCRs must be written for } \\
\text { the same purpose and product category } \\
\text { Both PCRs mandate a } \\
\text { Functional/Declared Unit which is the } \\
\text { same, or easily convertible - EPDs which } \\
\text { use different units } \\
\text { Both PCRs mandate the same set of } \\
\text { system boundaries - PCRs must have } \\
\text { the same processes within the system } \\
\text { boundaries scope } \\
\text { Both PCRs mandate the same cut-off } \\
\text { rules - PCRs that exclude different } \\
\text { amounts of impacts are not comparable } \\
\text { Both PCRs mandate the same temporal, } \\
\text { technological, and geographic data } \\
\text { requirements - data must be identical to } \\
\text { be sure it is comparable } \\
\text { Both PCRs mandate the same allocation } \\
\text { rules - data must be identical to be sure it } \\
\text { is comparable } \\
\text { Both PCRs mandate the same Life Cycle } \\
\text { Impact Assessment requirements, } \\
\text { including: } \\
\text { a) Inventory indicators } \\
\text { b) Impact categories } \\
\text { c) LCIA Characterization and } \\
\text { Weighting Factors } \\
\text { - PCRs are incomparable if the } \\
\text { quantified environmental impacts, the } \\
\text { parameters of which are described } \\
\text { above, are presented in different ways }\end{array}$ & $\begin{array}{l}\text { Both PCRs mandate an equivalent method for } \\
\text { primary data collection - may have some effect } \\
\text { on the quantified environmental impact data } \\
\text { within the resulting EPDs } \\
\text { Both PCRs either: } \\
\text { a) Have the same mandated use of } \\
\text { equivalent data sources, or; } \\
\text { b) Provide the criteria to determine which } \\
\text { data sources are appropriate, and the } \\
\text { criteria in both PCRs is the equivalent. } \\
\text { - PCRs must use similar data sources to } \\
\text { ensure the generic data used is comparable } \\
\text { Both PCRs mandate the use of an equivalent } \\
\text { system of measurement (IP v. SI) - PCRs which } \\
\text { use different systems of measurement need to } \\
\text { be converted to compared } \\
\text { Both PCRs mandate the statement of equivalent } \\
\text { Additional Environmental Information - some } \\
\text { PCRs may not include the same information as } \\
\text { others, and this should be taken into account } \\
\text { when comparing } \\
\text { Both PCRs use the same LCA type: either } \\
\text { attributional or consequential - may have effects } \\
\text { on the results based on the different approaches } \\
\text { of the two LCA types } \\
\text { Both PCRs adhere to the same sets of standards } \\
\text { - standards may have differing approaches to } \\
\text { requirements, structure, and formatting } \\
\text { Both PCRs mandate that manufacturing data to } \\
\text { be specific is a requirement - the resulting EPDs } \\
\text { that use generic manufacturing are unreliable } \\
\text { when compared to specific data }\end{array}$ \\
\hline
\end{tabular}

Table 32: Summarization Factors in EPD Comparison Matrix

Essentially, the harmonization between two PCRs does not exist if there is a "no" present in any of the crucial categories. The crucial categories provide a level of caution that should be implemented when judging the level of harmonization between PCRs. This section is entirely automated, according to the cell values present in the Comparison section. A sample of this component of the matrix is shown on the following page in Table 34. 


\begin{tabular}{|c|c|c|c|c|}
\hline SחOI $\perp \cap \forall J$ & & $\stackrel{\circ}{\subseteq}$ & $\stackrel{\Perp}{>}$ & $\stackrel{\Perp}{\nu}$ \\
\hline 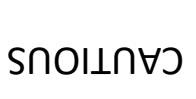 & & $\stackrel{\Perp}{\supset}$ & $\stackrel{\Perp}{>}$ & $\stackrel{\tilde{\nu}}{>}$ \\
\hline 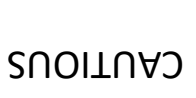 & & $\stackrel{\circ}{\subseteq}$ & $\stackrel{\varrho}{\nu}$ & $\stackrel{\check{\nu}}{\nu}$ \\
\hline 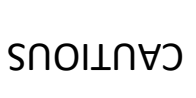 & & $\stackrel{\circ}{\complement}$ & 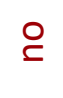 & $\stackrel{ }{\complement}$ \\
\hline SחOI $\perp \cap \forall J$ & SIINก & $\stackrel{\Perp}{>}$ & $\stackrel{\nu}{>}$ & $\stackrel{\mathscr{d}}{>}$ \\
\hline 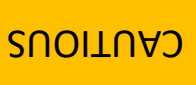 & SzJynOS $\forall \perp \forall 0$ & $\stackrel{\circ}{\subseteq}$ & 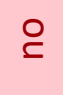 & 읃 \\
\hline SחOI $\perp \cap \forall J$ & 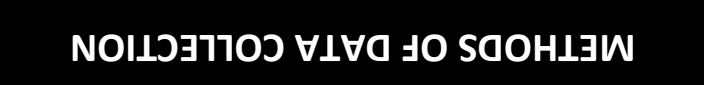 & $\stackrel{\circ}{\complement}$ & $\stackrel{\circ}{\simeq}$ & $\stackrel{\circ}{\complement}$ \\
\hline 7ซIวกษว & 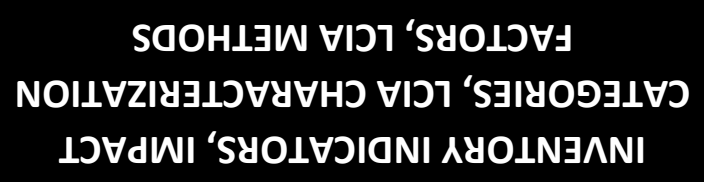 & ᄃ & $\stackrel{\circ}{\subseteq}$ & ב \\
\hline 7ซIวกษว & 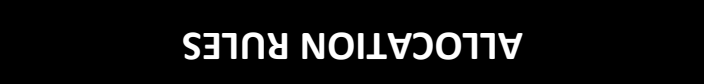 & $\stackrel{\circ}{\complement}$ & 옫 & $\stackrel{ }{\subseteq}$ \\
\hline 7ซIวกษว & 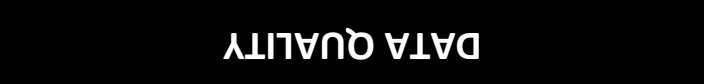 & $\stackrel{\circ}{\subseteq}$ & $\stackrel{\circ}{\subseteq}$ & 읃 \\
\hline 7ซIวกบว & Sang & $\stackrel{\circ}{\subseteq}$ & $\stackrel{\circ}{\subseteq}$ & 工 \\
\hline 7ซIวกษว & AyVONnOg WELSAS & $\stackrel{\circ}{\subseteq}$ & 옫 & $\stackrel{0}{\complement}$ \\
\hline 7ళIวกษว & IINก TVNOILJNก! & $\subseteq$ & ב & 工 \\
\hline 7ซIวกyว & 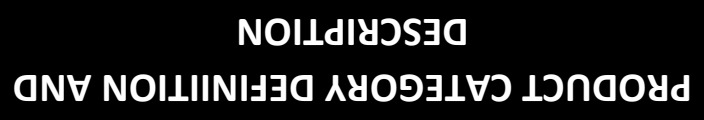 & U & $\stackrel{\tilde{\nu}}{\nu}$ & W \\
\hline
\end{tabular}




\subsubsection{Crucial Categories}

\subsubsection{Product Category Definition and Description}

This column inquires whether the product category definition and description in both PCRs being compared are the same. This is described in full in Sections 5.1.1 and 5.2.1. Two PCRs written for different purposes (e.g. a construction product PCR and a coffee bean PCR) are not directly comparable.

A "yes" is automatically inserted if the product category definition and description in both PCRs are the same; a "no" is automatically inserted if they differ.

\subsubsection{Functional/Declared Unit}

This column directly reflects the Functional/Declared Unit column, described in Sections 5.1.2 and 5.2.2. The functional/declared unit is required to be identical in ISO 14025, and can greatly affect the comparison process. For example, a comparison of insulation, where one EPD uses 1 $\mathrm{m}^{2}$ of insulation material with a thickness that gives a design thermal resistance of $1 \mathrm{~m}^{2} \cdot \mathrm{K} / \mathrm{W}$, and the other which uses $1 \mathrm{~m}^{3}$ of insulation material, cannot be compared (without the addition of supplementary material to convert, not available in many cases).

A "yes" is automatically inserted if the functional or declared units mandated in both PCRs being compared are the same. A "no" is automatically inserted if the two PCRs being compared do not have the same functional or declared unit.

\subsubsection{System Boundaries}

This column summarizes the results from all columns within the System Boundary section, discussed in Sections 5.1.3 and 5.2.3. The description of the elements within each life cycle module (i.e. Raw Material Acquisition Stage, Production Stage, etc.) must be identical for comparison as per ISO 14025. Two PCRs using two different system boundaries for the same stage are not comparable as they generate results for differing processes.

A "yes" is automatically generated if both PCRs being compared have identical system boundaries. A "no" is automatically generated if at least one of the system boundaries in both PCRs being compared are not identical. 


\subsubsection{Data Quality Requirements}

This column is a summarization of three columns in the Data Quality Requirements section, the Temporal, Technological, and Geographic Requirements described in Sections 5.1.4.1 and 5.2.4.1. A "yes" is automatically generated if both PCRs being compared have the same requirements for each column. A "no" is automatically generated if at least one of the requirements in the three columns in both PCRs being compared is not identical.

\subsubsection{Cut-Off Rules}

This column inquires whether the cut-off rules are the same in the two PCRs being compared, discussed in Sections 5.1.4.1 and 5.2.4.1. Cut-off rules crucial for comparison because each PCR allows the exclusion of different amounts of energy, mass, and environmental impact from LCA results, which directly affect the quantified environmental impacts in EPDs.

A "yes" is automatically generated if the two PCRs being compared have the same exact set of cut-off rules. A "no" is automatically generated if the two PCRs have different sets of cut-off rules.

\subsubsection{Allocation Rules}

This column focuses on the results from the Allocation Rules, discussed in Sections 5.1.4.1 and 5.2.4.1, and are required to be identical as per ISO 14025.

A "yes" is automatically generated if both PCRs being compared have the same allocation rules. A "no" is automatically generated if both PCRs being compared have two different sets of allocation rules.

\subsubsection{Life Cycle Impact Assessment Requirements}

This column summarizes the results from the three columns in the Life Cycle Impact Assessment section of the PCR Comparison Matrix, discussed in Sections 5.1.5 and 5.2.5. PCRs must use the same impact categories and characterization factors in order to be comparable, and are required to be identical as per ISO 14025.

A "yes" is automatically generated if both PCRs being compared have the same impact categories, impact indicators, normalization rules, and weighting rules. A "no" is automatically generated if at least one of the three columns differ between PCRs. 


\subsubsection{Cautious Categories}

\subsubsection{Same Standard Set}

This column is the result of two columns in the General Information section: whether the PCR is written to ISO 14025, and whether the PCR is written to EN 15804. This category requires caution because it has overarching effects on the comparison results such as different requirements and formatting techniques within the resulting EPDs.

A "yes" is automatically inserted if both PCRs being compared are written to the same set of standards (i.e. ISO 14025 but not EN 15804, or both ISO 14025 and EN 15804). A "no" is automatically inserted if the PCRs being compared are written to different standard sets.

\subsubsection{LCA Type}

This column is the reflection of the LCA Type column in the Goal and Scope Definition of LCA section, where the input is either "attributional" or "consequential". This would have some effect on data within resulting EPDs (attributional LCAs might be approached or conducted differently than consequential LCAs), but not enough to preclude the comparison. A "yes" is automatically inserted if both PCRs being compared mandate the same LCA type (i.e. both attributional, or both consequential). A "no" is automatically inserted if the two PCRs mandate different LCA types.

\subsubsection{Methods of Data Collection}

This column inquires whether the PCRs being compared use the same Method for Primary Data Collection. Different data collection methods may affect results of the LCA, but not enough to preclude the comparability of resulting EPDs (i.e. two different generic databases may have slightly different environmental impacts for background processes). A "yes" is automatically inserted if the two PCRs being compared use an equivalent method for primary data collection. A "no" is automatically inserted if the methods of data collection in the two PCRs are not equivalent.

\subsubsection{Specific Manufacturing Data}

This column uses the results from only one of the columns within the Primary and Secondary Data section, described in Sections 5.1.4.2 and 5.2.4.2. Specific data is much more accurate than generic data, and is generally required for stages within the manufacturer's control within international standards. Because of this, it is important to be aware of differences in the data used for reporting as this could significantly affect reported values. This column asks whether 
specific data is required for the Manufacturing Stage for both PCRs being compared. A "yes" is automatically inserted if it is required in both PCRs; a "no" is automatically inserted if it is not required in one or both of the $\mathrm{PCRs}$.

\subsubsection{Data Sources}

This column also focuses on the Primary and Secondary Data section, using the results from the Data Sources column. Using different databases for generic data can lead to differing results in the LCA, although not drastic, and may have some effect on the comparison. A "yes" is automatically inserted if both PCRs mandate the use of equivalent background data sources, or criteria in which they can be selected. A "no" is automatically inserted if the PCRs differ in this regard.

\subsubsection{Units}

This column reflects the results in the Units column in the Other Inputs section, described in Sections 5.1.5 and 5.2.5. A "yes" is automatically inserted if both PCRs being compared use the same system of measurement: either both IP, or both SI. A "no" is automatically inserted if the two PCRs being compared mandate different units.

\subsubsection{Additional Environmental Information}

This column is focused on the results from the Additional Environmental Information column in the Other Inputs section. PCRs which have differing additional environmental information would not state the same information in the resulting EPDs (e.g. VOC emissions, radioactivity, worker safety management, etc), but would not preclude comparison. A "yes" is automatically inserted if both PCRs have equivalent requirements for the statement of additional environmental information within the EPD. A "no" is automatically inserted if the requirements for additional environmental information are not equivalent.

\subsubsection{Hazardous Substances}

Similar to the last column in the previous section, this column focuses on the requirements for declaring hazardous substances within the EPD. A "yes" is automatically inserted if both PCRs being compared have equivalent

\subsubsection{Scoring}

The scoring for the PCR Comparison Matrix is in a very similar format to that of the EPD Comparison Matrix, described in Section 4.3.3. Table 35 below shows a typical PCR Comparison Matrix Scoring Section. 


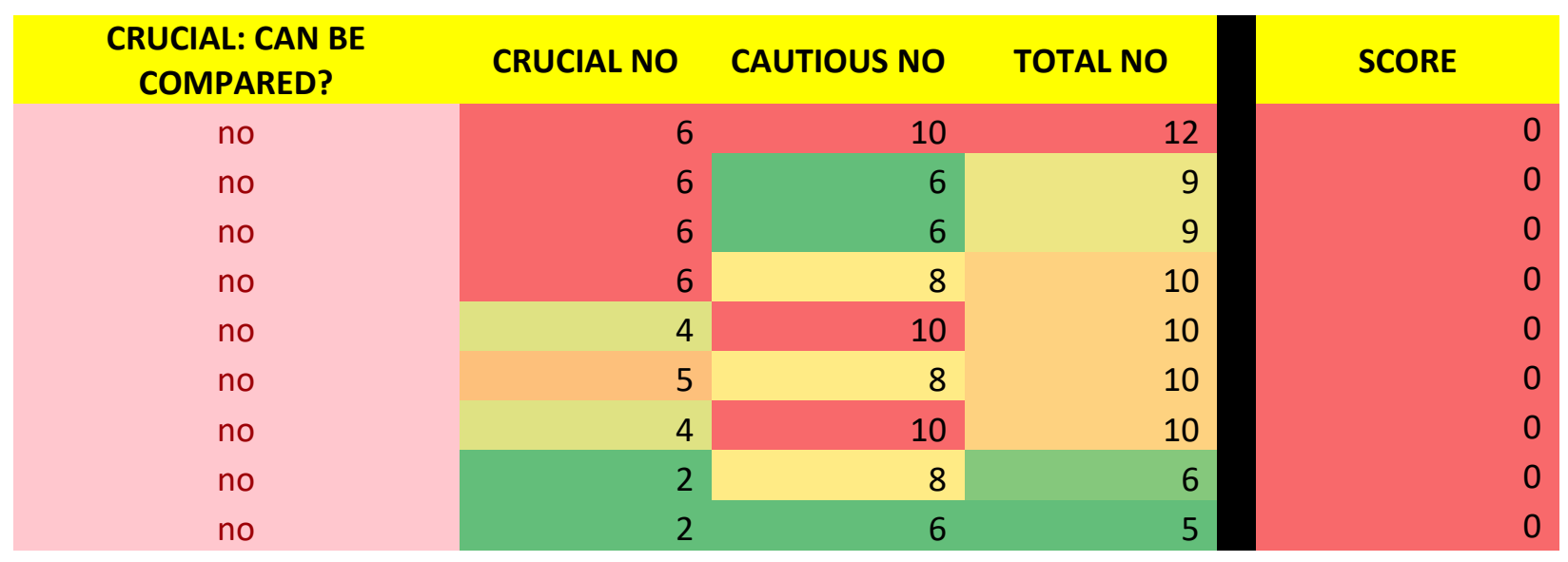

Table 34: Sample entries from PCR Comparison Matrix, Scoring section

The Scoring Component includes eight significant columns, two of which are not shown in the above table.

First, the "Crucial: Can be Compared?" column, is automatically generated based on the results of the Comparison section. A "yes" is generated if all the crucial categories (from Section 6.3.1) are all "yes"s, which essentially means the EPDs can be compared according to ISO 14025 6.7.2 Requirements for comparability. A "no" is generated if at least one of the crucial categories contains a "no".

The three succeeding columns, titled "Crucial No", "Cautious No", and "Total No" quantify the results from the Comparison section. The "Crucial No" column quantifies the amount of "no"s from the elements in Section 5.3.1, and the "Cautious No" column quantifies the amount of "no"s from the elements in Section 5.3.2. The "Total No" column is a sum of two prior columns.

The "Score" column scores the EPDs based on their comparability. It starts at 20, and is subtracted from based on the amount of "Crucial No"s and "Cautious No"s the comparison has. A "Crucial No" brings the score to 0, meaning the two EPDs cannot be compared. A "Cautious No" subtracts 1 or 2 points from the score, depending on the effect it has on the validity of the comparison. 


\section{Using the Environmental Product Declaration Comparison Matrix}

This section describes the process of evaluating whether two EPDs are comparable in identified product categories related to construction projects. Outlined in this section is the reasoning behind the selection of the product categories, the method used to complete the EPD Comparison Matrix, and the key results and discussion arising from the completion of the matrix.

\subsection{Selection of Categories for Comparison}

Three product categories were chosen for comparison: insulation, carpet, and cladding. Insulation was chosen because of its important function in the North American building envelope, and the fact that there are many varieties in the insulation product category. This could give a sense as to whether or not comparability was achieved throughout the whole product category (all insulating materials) or within sub-categories (stone wool, and foam/polymer based insulating materials). Insulation also includes a range of products that could be substituted during the design phase with significant differences if the team is interested in minimizing environmental impact.

Carpet manufacturers have been pioneers in collecting data and creating LCAs because of their potential to release volatile organic compounds (VOCs). As such, it is expected that the EPDs within the carpet product category will be more consistent than other categories, and therefore have more opportunity for comparability between products.

The cladding product category focuses on "metal panels with insulating material", which includes sandwich panels and insulated back-up panels. A category of a composite material or an assembly of different materials was desired for comparison. This category will theoretically include products with much more variety in material content and distribution than the other two categories. Furthermore, the addition of this category provides a realistic scope for a building project; the comparison considers materials on the interior (carpet), within the envelope (insulation), and on the exterior (cladding).

EPDs were found using two methods. To begin, products with EPDs were sourced using databases from larger program operators, such as Underwriters Laboratory Environment (UL Environment), NSF International, Institut Bauen und Umwelt (IBU), The International EPD System (Environdec), and others. This established a proportion of products from program 
operators that design practitioners would almost certainly be familiar with when using EPDs on a construction project. Following this, other EPDs were found through online searches using keywords related to EPDs and the product category being searched for. This opened up the library's selection to EPDs that are not on a program operator's database, EPDs from program operators who do not have a database, and EPDs that do not have a program operator. This allowed a much broader range of products to be compiled than would have been identified using only using the first method.

\subsubsection{Insulation}

Insulation products found can be broken down into the following sub-categories:

- Stone wool, both board and loose-fill

- Foam and polymers, including:

o Extruded polystyrene (XPS)

o Expanded polystyrene (EPS)

o Polyisocyanurate board

o Spray foam

The varying raw material distribution along this product category does not affect the comparison. The metrics of the comparison are focused on the content included in the EPD, not the quantified environmental impacts of the products. They are all in the same product category as they all fulfill equivalent functions (building thermal insulation), as established by ISO 14025.

12 products were chosen for comparison, characterized by 12 different manufacturers or product associations, 6 different program operators, and 5 different PCRs.

\subsubsection{Carpet}

14 products were chosen for comparison, 11 of which are tiled systems and 3 of which are broadloom. In this section, there are 11 different manufacturers, 4 different program operators, and 3 different PCRs.

\subsubsection{Cladding}

All products in the cladding EPD Comparison Matrix are metal-clad panels with insulating material. Most of the cladding systems in this category are sandwich panels, with 1 insulated back-up panel included. The sandwich panels have varying insulating materials within them, including foams and stone wool. 14 products were chosen for comparison, characterized by 12 
different manufacturers or product associations, 4 different program operators, and 5 different PCRs.

\subsection{Comparison Methodology}

The EPD Comparison Matrix was completed using the chart as described in Section 5. The chart was completed row by row by choosing an EPD and filling in all the required information along the row of the Inputs section, rather than column by column.

One caveat in the EPD Comparison Matrix Inputs section was the fact that cut-off rules were sometimes not explicitly stated within the declaration. The EPD either: disclosed the entire cutoff rules, disclosed that cut-off rules were not applied, or did not discuss cut-off rules whatsoever. For this column, the cut-off rules were sourced from the PCR itself where it was not disclosed in the EPD.

Following this, the Comparison section was completed, using results from the Inputs section. Factorial comparisons were constructed in order to compare every EPD within the matrix to every other EPD the template was populated with. Either a "yes" or "no" was inserted for each factorial comparison based on the information within the EPD. Some sections required a "yes" or "no" to be manually determined based on both EPDs both including information. An example of this is in the System Boundaries category, where the two EPDs being compared are required to declare the same system boundary (both declare Transport to Landfill, "yes"). Other scenarios are more precise such as in the Data Quality Requirements section, where both EPDs being compared have to use specific data in order to get a "yes".

The Summarization section, and therefore the Scoring section is automatically calculated when the "yes" and "no" cells are completed within the Comparison section. EPDs that can be compared can then be seen at the culmination of the Scoring section.

\subsection{Results and Discussion}

\subsubsection{Comparison of Crucial Content}

There are five elements of Crucial Content in the EPD Comparison Matrix. The extent of their comparability is summarized in Figure 6 below and will be discussed in the following sections. 


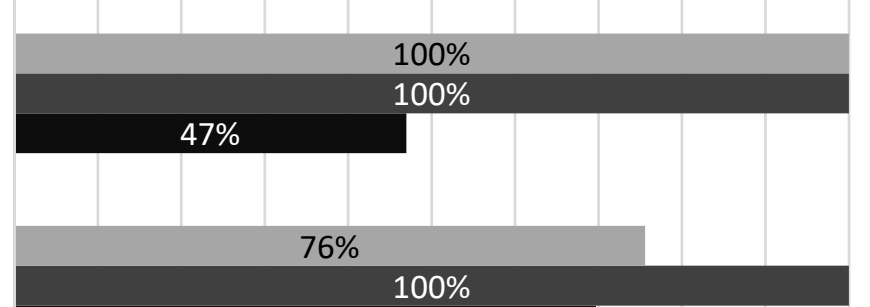

SYSTEM BOUNDARIES (SEPARATED OR SAME) COMPARABLE

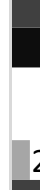

IMPACT CATEGORY CHARACTERIZATION FACTORS

CUT-OFF RULES DEFINED OR IDENTICAL

MANDATORY CONTENT FOR COMPARISON PRESENT

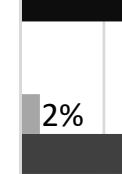

$70 \%$

\section{$12 \%$}

$12 \%$

$32 \%$

$49 \%$
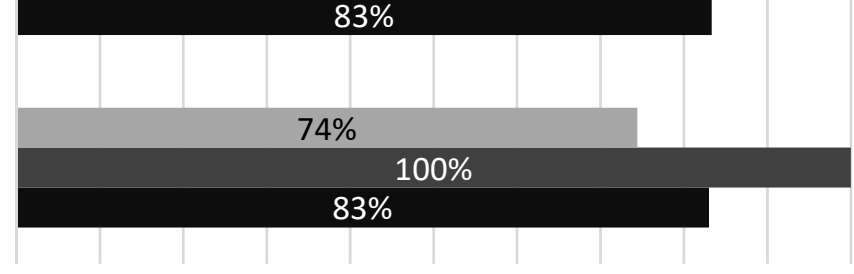

$\begin{array}{lllllllllll}0 \% & 10 \% & 20 \% & 30 \% & 40 \% & 50 \% & 60 \% & 70 \% & 80 \% & 90 \% & 100 \%\end{array}$

Cladding Flooring Insulation

Figure 6: EPD Comparison Matrix Crucial Comparison Results

\subsubsection{Same Functional/Declared Unit}

There seems to be a difference in the Same Functional/Declared unit category, with less than half of the comparisons passing in the Insulation category, while the two other product categories are consistent. In the Flooring category, each EPD uses $1 \mathrm{~m}^{2}$ of flooring area covered, or a similar descriptor. In the Cladding category, the functional or declared units were not as consistent, but all had units that could allow the environmental impact results to be converted and compared on an area basis: $1000 \mathrm{ft}^{2}, 100 \mathrm{ft}^{2}$, or $1 \mathrm{~m}^{2}$. This is not the case in the Insulation category. Essentially, the difference is because of the characteristics of insulation as a construction material: its thickness can vary widely dependent on the manufacturing process of the product and its use in a building context. Some Insulation EPDs used functional or declared units of $1 \mathrm{~m}^{2}$ of insulation material with a thickness that gives a performance of 1 $\mathrm{m}^{2} \cdot \mathrm{K} / \mathrm{W}$, while others used units of $1 \mathrm{~m}^{3}$. At times, the thickness of the material that gives this performance is not stated, and can preclude comparison if it cannot be converted to an equivalent. 


\subsubsection{System Boundaries Separated or Same}

The Flooring category was very consistent in regards to the System Boundaries used in the EPDs, and all of the EPDs analyzed separated their system boundaries. In the Insulation Category, there was a large variation in the actual system boundaries that were declared, and only two of the EPDs had separated their impacts by system boundaries. Table 36 below illustrates the difference in system boundaries declared across all Insulation EPDs analyzed. A similar trend is present in the Cladding EPDs analyzed.

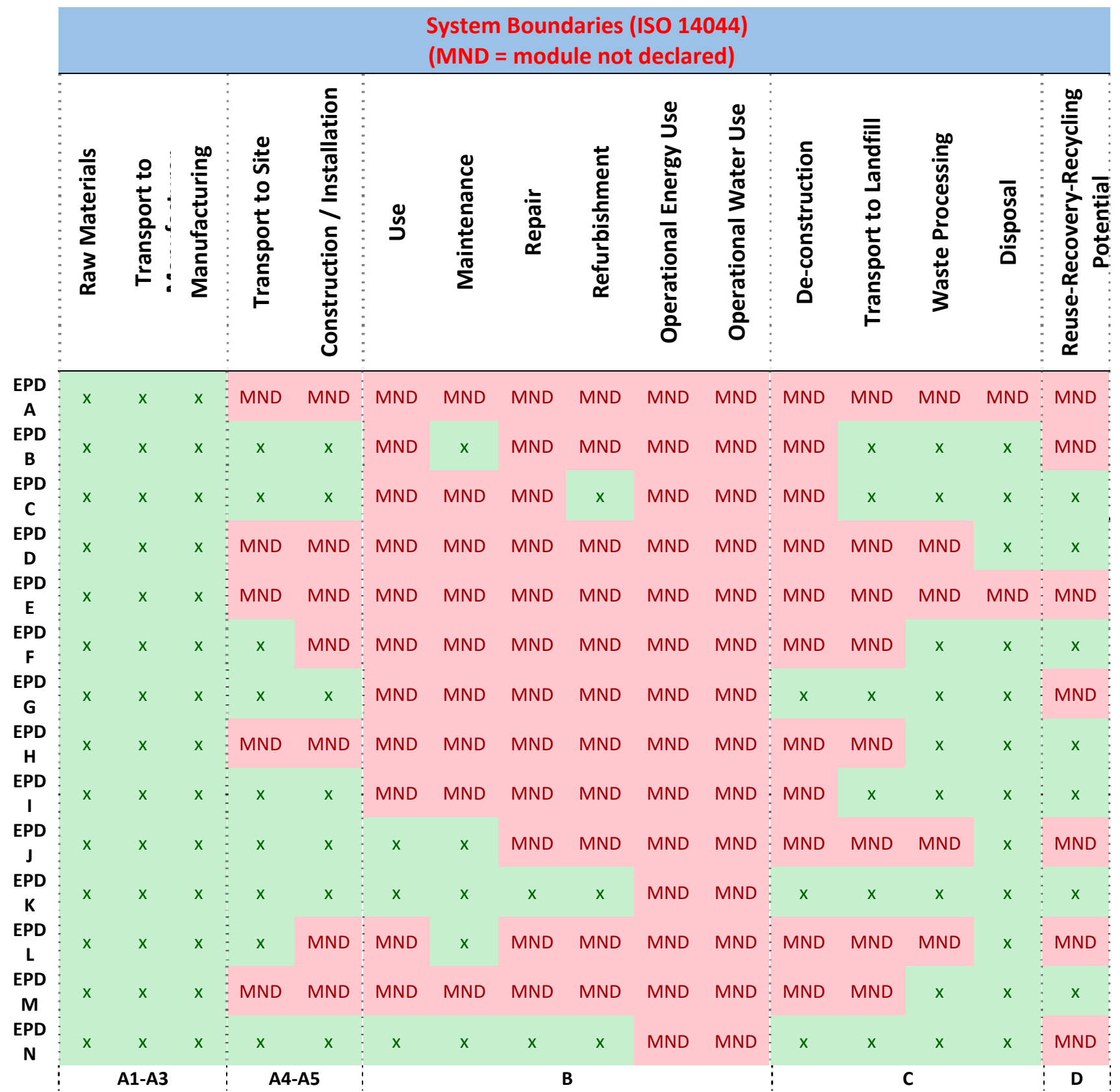

Table 35: System Boundary Declarations in Insulation EPDs 
Only two comparisons here have identical system boundaries: $A$ and $E$, and $H$ and $M$. However, if we look at system boundaries by separated modules (A1-A3, A4-A5, B, C, and D, as discussed in Section 5.1.3 and illustrated in the chart above), it becomes evident that more comparisons can be validated if results are available for each individual module. A crucial column in this category, titled "Are Impacts Separated by System Boundaries?" allows for comparisons to be made as long as impacts are separated by module, therefore allowing comparison between modules that are the same (and putting less importance on, or ignoring modules that do not match). The results for this column in each section are Insulation with $70 \%$ of comparisons Flooring $100 \%$, and Cladding $74 \%$.

\subsubsection{Impact Category Characterization Factors}

The category that has the most inherent deficiencies is the Impact Category Characterization Factors. In this case, EPDs will fail this for one or both of the following reasons:

1. EPDs declare different characterization factors in different regions. This phenomenon was discussed in Section 4.1.4 Impact Categories, where acidification is identified using either $\mathrm{kg} \mathrm{SO}_{2}$ eq (sulfur dioxide), largely used in Europe, or mol $\mathrm{H}+$ eq (hydrons), largely used in North America. Eutrophication is identified using either $\mathrm{kg} \mathrm{PO}_{4}{ }^{3-} \mathrm{eq}$ (phosphate), Europe, or kg N eq (nitrogen), North America. This was seen mostly in the Insulation category. In Table 37 below, asterisks beside characterization factors meant they were able to be converted. 


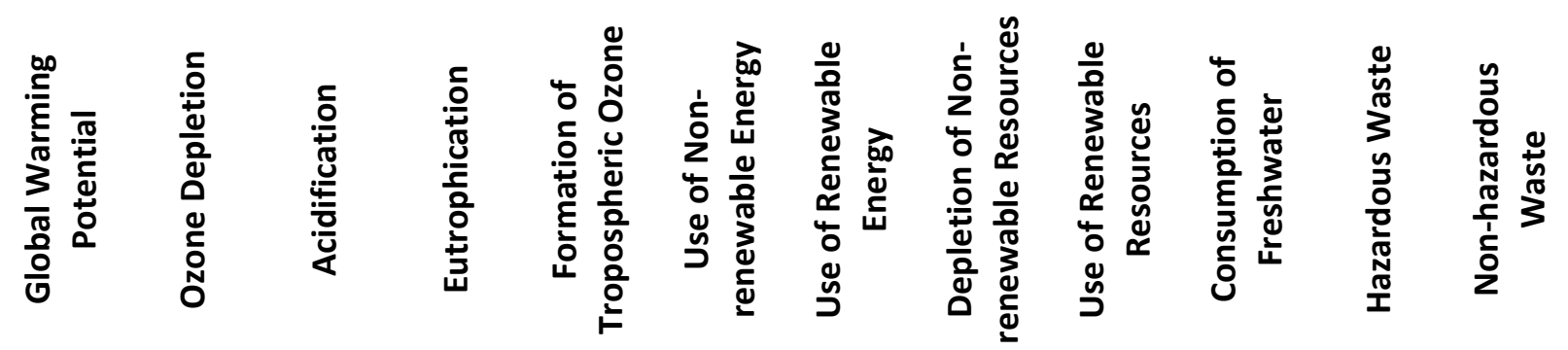

\begin{tabular}{|c|c|c|c|c|c|c|c|c|c|c|c|}
\hline $\begin{array}{c}\mathrm{kg} \mathrm{CO}_{2} \\
\text { eq }\end{array}$ & $\begin{array}{c}\text { kg CFC- } \\
11 \text { eq }\end{array}$ & $\begin{array}{c}\mathrm{kg} \mathrm{SO}_{2} \\
\text { eq }\end{array}$ & $\begin{array}{c}\mathrm{kg} \mathrm{PO}_{4} \\
\text { eq }\end{array}$ & $\begin{array}{c}\mathrm{kg} \\
\mathrm{C} 2 \mathrm{H} 4\end{array}$ & MJ* & MJ* & & & $\mathrm{m}^{3}$ & $\mathrm{~kg}$ & $\mathrm{~kg}$ \\
\hline $\begin{array}{c}\mathrm{kg} \mathrm{CO}_{2} \\
\text { eq }\end{array}$ & $\begin{array}{c}\text { kg CFC- } \\
11 \text { eq }\end{array}$ & $\begin{array}{c}\mathrm{mol} \mathrm{H}^{\mathrm{H}} \\
\mathrm{eq}^{*}\end{array}$ & $\begin{array}{l}\mathrm{kg} \mathrm{N} \\
\mathrm{eq}^{*}\end{array}$ & & MJ & $\mathrm{MJ}$ & MJ & MJ & Gal* & & $\mathrm{kg}$ \\
\hline $\begin{array}{c}\mathrm{kg} \mathrm{CO}_{2} \\
\text { eq }\end{array}$ & $\begin{array}{c}\text { kg CFC- } \\
11 \text { eq }\end{array}$ & $\begin{array}{c}\mathrm{kg} \mathrm{SO}_{2} \\
\text { eq }\end{array}$ & $\begin{array}{c}\mathrm{kg} \mathrm{PO}_{4} \\
\text { eq }\end{array}$ & $\begin{array}{c}\mathrm{kg} \\
\mathrm{C} 2 \mathrm{H} 4\end{array}$ & MJ & MJ & MJ & MJ & $\mathrm{m}^{3}$ & $\mathrm{~kg}$ & $\mathrm{~kg}$ \\
\hline $\begin{array}{c}\mathrm{kg} \mathrm{CO}_{2} \\
\text { eq }\end{array}$ & $\begin{array}{c}\text { kg CFC- } \\
11 \text { eq }\end{array}$ & $\begin{array}{c}\mathrm{kg} \mathrm{SO}_{2} \\
\mathrm{eq}\end{array}$ & $\begin{array}{c}\mathrm{kg} \mathrm{PO}_{4} \\
\text { eq }\end{array}$ & $\begin{array}{c}\mathrm{kg} \\
\text { Ethen } \\
\text { eq }\end{array}$ & MJ & $\mathrm{MJ}$ & MJ & MJ & $\mathrm{m}^{3}$ & $\mathrm{~kg}$ & $\mathrm{~kg}$ \\
\hline $\begin{array}{c}\mathrm{kg} \mathrm{CO}_{2} \\
\text { eq }\end{array}$ & $\begin{array}{l}\text { kg CFC- } \\
11 \text { eq }\end{array}$ & $\begin{array}{c}\mathrm{kg} \mathrm{SO}_{2} \\
\text { eq }\end{array}$ & $\begin{array}{c}\mathrm{kg} \mathrm{PO}_{4} \\
\text { eq }\end{array}$ & $\begin{array}{c}\mathrm{kg} \\
\text { Ethen } \\
\text { eq }\end{array}$ & MJ & $\mathrm{MJ}$ & MJ & MJ & $\mathrm{m}^{3}$ & $\mathrm{~kg}$ & $\mathrm{~kg}$ \\
\hline $\begin{array}{c}\mathrm{kg} \mathrm{CO}_{2} \\
\text { eq }\end{array}$ & $\begin{array}{c}\text { kg CFC- } \\
11 \text { eq }\end{array}$ & $\begin{array}{c}\mathrm{kg} \mathrm{SO}_{2} \\
\mathrm{eq}\end{array}$ & $\begin{array}{c}\mathrm{kg} \mathrm{PO}_{4} \\
\text { eq }\end{array}$ & $\begin{array}{c}\mathrm{kg} \\
\mathrm{C} 2 \mathrm{H} 4\end{array}$ & MJ & $\mathrm{MJ}$ & MJ & MJ & $\mathrm{m}^{3}$ & $\mathrm{~kg}$ & $\mathrm{~kg}$ \\
\hline $\begin{array}{c}\mathrm{kg} \mathrm{CO}_{2} \\
\text { eq }\end{array}$ & $\begin{array}{l}\text { kg CFC- } \\
11 \text { eq }\end{array}$ & $\begin{array}{c}\mathrm{kg} \mathrm{SO}_{2} \\
\text { eq }\end{array}$ & $\begin{array}{c}\mathrm{kg} \mathrm{PO}_{4} \\
\text { eq }\end{array}$ & $\begin{array}{c}\mathrm{kg} \\
\text { Ethen } \\
\text { eq }\end{array}$ & MJ & $\mathrm{MJ}$ & MJ & MJ & $\mathrm{m}^{3}$ & $\mathrm{~kg}$ & $\mathrm{~kg}$ \\
\hline $\begin{array}{c}\mathrm{kg} \mathrm{CO}_{2} \\
\text { eq }\end{array}$ & $\begin{array}{c}\text { kg CFC- } \\
11 \text { eq }\end{array}$ & $\begin{array}{c}\mathrm{kg} \mathrm{SO}_{2} \\
\text { eq }\end{array}$ & $\begin{array}{c}\mathrm{kg} \mathrm{PO}_{4} \\
\text { eq }\end{array}$ & $\begin{array}{c}\mathrm{kg} \\
\text { Ethen } \\
\text { eq }\end{array}$ & MJ & $\mathrm{MJ}$ & MJ & MJ & $\mathrm{m}^{3}$ & $\mathrm{~kg}$ & $\mathrm{~kg}$ \\
\hline $\begin{array}{c}\mathrm{kg} \mathrm{CO}_{2} \\
\text { eq }\end{array}$ & $\begin{array}{c}\text { kg CFC- } \\
11 \text { eq }\end{array}$ & $\begin{array}{c}\mathrm{mol} \mathrm{H}^{+} \\
\text {eq }^{*}\end{array}$ & $\begin{array}{l}\mathrm{kg} \mathrm{N} \\
\text { eq* }\end{array}$ & & MJ & $\mathrm{MJ}$ & & & $\mathrm{kg}$ & $\mathrm{kg}$ & $\mathrm{kg}$ \\
\hline $\begin{array}{c}\mathrm{kg} \mathrm{CO}_{2} \\
\text { eq }\end{array}$ & $\begin{array}{c}\text { kg CFC- } \\
11 \text { eq }\end{array}$ & $\begin{array}{c}\mathrm{mol} \mathrm{H}^{+} \\
\mathrm{eq}^{*}\end{array}$ & $\begin{array}{l}\mathrm{kg} \mathrm{N} \\
\text { eq* }\end{array}$ & & & & & & L & & \\
\hline $\begin{array}{c}\mathrm{kg} \mathrm{CO}_{2} \\
\text { eq }\end{array}$ & $\begin{array}{c}\text { kg CFC- } \\
11 \text { eq }\end{array}$ & $\begin{array}{c}\mathrm{kg} \mathrm{SO}_{2} \\
\text { eq }\end{array}$ & $\begin{array}{c}\mathrm{kg} \mathrm{PO}_{4} \\
\text { eq }\end{array}$ & $\begin{array}{c}\mathrm{kg} \\
\mathrm{C} 2 \mathrm{H} 2 \\
\text { eq }\end{array}$ & MJ & $\mathrm{MJ}$ & MJ & MJ & L & $\mathrm{kg}$ & $\mathrm{kg}$ \\
\hline $\begin{array}{c}\mathrm{kg} \mathrm{CO}_{2} \\
\mathrm{eq}\end{array}$ & $\begin{array}{c}\text { kg CFC- } \\
11 \text { eq }\end{array}$ & $\begin{array}{c}\mathrm{kg} \mathrm{SO}_{2} \\
\mathrm{eq}\end{array}$ & $\begin{array}{c}\mathrm{kg} \mathrm{PO}_{4} \\
\text { eq }\end{array}$ & $\begin{array}{c}\mathrm{kg} \mathrm{C}_{2} \mathrm{H}_{4} \\
\mathrm{eq}\end{array}$ & MJ & $\mathrm{MJ}$ & MJ & MJ & $\mathrm{m}^{3}$ & $\mathrm{~kg}$ & $\mathrm{~kg}$ \\
\hline
\end{tabular}

Table 36: Characterization Factors in Insulation EPDs

2. Some EPDs fail to include the one or multiple of the categories outlined in Section 5.1.4 Impact Categories. In all EPDs that were analyzed, global warming potential, acidification, and eutrophication are always declared. The problems occur in the other impact categories, especially the non-renewable and renewable resources categories. This was seen mostly in the Cladding (shown in Table 38), and to a lesser extent, the Flooring categories. 
Impact Categories (ISO 21930 8.2.2)

\begin{tabular}{|c|c|c|c|c|c|c|c|c|c|c|c|}
\hline 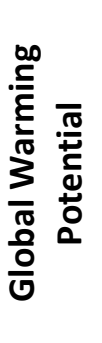 & 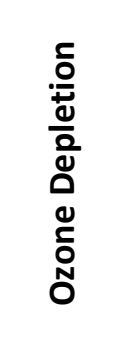 & 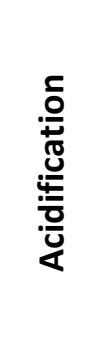 & $\begin{array}{l}\frac{}{0} \\
\frac{0}{0} \\
\frac{U}{2} \\
\frac{1}{0} \\
\frac{0}{0} \\
\frac{2}{2} \\
\end{array}$ & 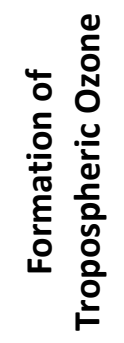 & 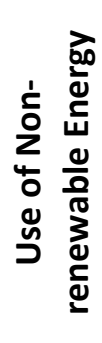 & 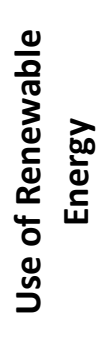 & 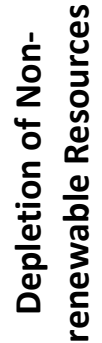 & 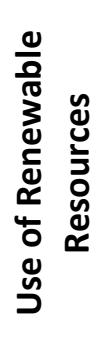 & 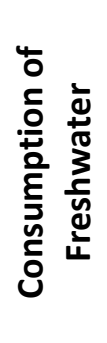 & 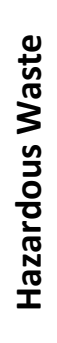 & 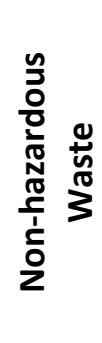 \\
\hline $\begin{array}{l}\mathrm{kg} \mathrm{CO}_{2} \\
\text { eq }\end{array}$ & $\begin{array}{c}\text { kg CFC- } \\
11 \text { eq }\end{array}$ & $\begin{array}{c}\mathrm{kg} \mathrm{SO}_{2} \\
\text { eq }\end{array}$ & $\begin{array}{c}\mathrm{kg} \mathrm{PO}_{4} \\
\text { eq }\end{array}$ & $\begin{array}{c}\mathrm{kg} \mathrm{C}_{2} \mathrm{H}_{4} \\
\text { eq }\end{array}$ & MJ & MJ & & & $\mathrm{m}^{3}$ & $\mathrm{~kg}$ & $\mathrm{Kg}$ \\
\hline $\begin{array}{c}\mathrm{kg} \mathrm{CO}_{2} \\
\text { eq }\end{array}$ & $\begin{array}{c}\text { kg R-11 } \\
\text { eq }\end{array}$ & $\begin{array}{c}\mathrm{kg} \mathrm{SO}_{2} \\
\mathrm{eq}\end{array}$ & $\begin{array}{c}\mathrm{kg} \mathrm{PO}_{4} \\
\text { eq }\end{array}$ & $\begin{array}{c}\mathrm{kg} \mathrm{C}_{2} \mathrm{H}_{4} \\
\text { eq }\end{array}$ & MJ & MJ & & & $\mathrm{m}^{3}$ & & \\
\hline $\begin{array}{c}\mathrm{kg} \mathrm{CO}_{2} \\
\text { eq }\end{array}$ & $\begin{array}{c}\text { kg CFC- } \\
11 \text { eq }\end{array}$ & $\begin{array}{c}\mathrm{kg} \mathrm{SO}_{2} \\
\text { eq }\end{array}$ & $\begin{array}{c}\mathrm{kg} \mathrm{PO}_{4} \\
\text { eq }\end{array}$ & $\begin{array}{c}\mathrm{kg} \mathrm{C}_{2} \mathrm{H}_{4} \\
\text { eq }\end{array}$ & MJ & MJ & MJ & MJ & $\mathrm{m}^{3}$ & $\mathrm{~kg}$ & kg \\
\hline $\begin{array}{l}\mathrm{kg} \mathrm{CO}_{2} \\
\mathrm{eq}\end{array}$ & $\begin{array}{c}\text { kg CFC- } \\
11 \text { eq }\end{array}$ & $\begin{array}{c}\mathrm{kg} \mathrm{SO}_{2} \\
\mathrm{eq}\end{array}$ & $\begin{array}{c}\mathrm{kg} \mathrm{PO}_{4} \\
\text { eq }\end{array}$ & $\begin{array}{c}\mathrm{kg} \mathrm{C}_{2} \mathrm{H}_{4} \\
\mathrm{eq}\end{array}$ & MJ & MJ & MJ & MJ & & & \\
\hline $\begin{array}{c}\mathrm{kg} \mathrm{CO} 2 \\
\text { eq }\end{array}$ & $\begin{array}{c}\text { kg CFC- } \\
11 \text { eq }\end{array}$ & $\begin{array}{c}\mathrm{kg} \mathrm{SO}_{2} \\
\text { eq }\end{array}$ & $\begin{array}{c}\mathrm{kg} \mathrm{PO}_{4} \\
\text { eq }\end{array}$ & $\begin{array}{c}\mathrm{kg} \mathrm{C}_{2} \mathrm{H}_{4} \\
\text { eq }\end{array}$ & MJ & MJ & & & $\mathrm{m}^{3}$ & $\mathrm{~kg}$ & $\mathrm{~kg}$ \\
\hline $\begin{array}{c}\mathrm{kg} \mathrm{CO}_{2} \\
\text { eq }\end{array}$ & $\begin{array}{c}\text { kg CFC- } \\
11 \text { eq }\end{array}$ & $\begin{array}{c}\mathrm{kg} \mathrm{SO}_{2} \\
\text { eq }\end{array}$ & $\begin{array}{c}\mathrm{kg} \mathrm{PO}_{4} \\
\text { eq }\end{array}$ & $\begin{array}{c}\mathrm{kg} \mathrm{C}_{2} \mathrm{H}_{4} \\
\text { eq }\end{array}$ & MJ & MJ & MJ & MJ & $\mathrm{m}^{3}$ & $\mathrm{~kg}$ & $\mathrm{~kg}$ \\
\hline $\begin{array}{c}\mathrm{kg} \mathrm{CO}_{2} \\
\text { eq }\end{array}$ & $\begin{array}{c}\text { kg CFC- } \\
11 \text { eq }\end{array}$ & $\begin{array}{c}\mathrm{kg} \mathrm{SO}_{2} \\
\text { eq }\end{array}$ & $\begin{array}{c}\mathrm{kg} \mathrm{PO}_{4} \\
\text { eq }\end{array}$ & $\begin{array}{c}\mathrm{kg} \\
\text { NMVOC }\end{array}$ & & & & & & & \\
\hline $\begin{array}{c}\mathrm{kg} \mathrm{CO}_{2} \\
\text { eq }\end{array}$ & $\begin{array}{c}\text { kg CFC- } \\
11 \text { eq }\end{array}$ & $\begin{array}{c}\mathrm{kg} \mathrm{SO}_{2} \\
\mathrm{eq}\end{array}$ & $\begin{array}{c}\mathrm{kg} \mathrm{PO}_{4} \\
\text { eq }\end{array}$ & $\begin{array}{c}\mathrm{kg} \mathrm{C}_{2} \mathrm{H}_{4} \\
\text { eq }\end{array}$ & MJ & MJ & MJ & MJ & & & \\
\hline $\begin{array}{c}\mathrm{kg} \mathrm{CO} 2 \\
\text { eq }\end{array}$ & $\begin{array}{c}\text { kg CFC- } \\
11 \text { eq }\end{array}$ & $\begin{array}{c}\mathrm{kg} \mathrm{SO}_{2} \\
\text { eq }\end{array}$ & $\begin{array}{c}\mathrm{kg} \mathrm{PO}_{4} \\
\text { eq }\end{array}$ & $\begin{array}{c}\mathrm{kg} \mathrm{C}_{2} \mathrm{H}_{4} \\
\text { eq }\end{array}$ & MJ & MJ & MJ & MJ & & & \\
\hline $\begin{array}{c}\mathrm{kg} \mathrm{CO}_{2} \\
\text { eq }\end{array}$ & $\begin{array}{c}\text { kg CFC- } \\
11 \text { eq }\end{array}$ & $\begin{array}{c}\mathrm{kg} \mathrm{SO}_{2} \\
\text { eq }\end{array}$ & $\begin{array}{c}\mathrm{kg} \mathrm{PO}_{4} \\
\text { eq }\end{array}$ & $\begin{array}{c}\mathrm{kg} \mathrm{C}_{2} \mathrm{H}_{4} \\
\text { eq }\end{array}$ & MJ & MJ & & MJ & $\mathrm{m}^{3}$ & $\mathrm{~kg}$ & $\mathrm{~kg}$ \\
\hline $\begin{array}{c}\mathrm{kg} \mathrm{CO}_{2} \\
\text { eq }\end{array}$ & $\begin{array}{c}\text { kg CFC- } \\
11 \text { eq }\end{array}$ & $\begin{array}{c}\mathrm{kg} \mathrm{SO}_{2} \\
\mathrm{eq}\end{array}$ & $\begin{array}{c}\mathrm{kg} \mathrm{PO}_{4} \\
\text { eq }\end{array}$ & $\begin{array}{c}\mathrm{kg} \mathrm{C}_{2} \mathrm{H}_{4} \\
\text { eq }\end{array}$ & MJ & MJ & MJ & MJ & $\mathrm{m}^{3}$ & $\mathrm{~kg}$ & $\mathrm{~kg}$ \\
\hline $\begin{array}{c}\mathrm{kg} \mathrm{CO}_{2} \\
\text { eq }\end{array}$ & $\begin{array}{c}\text { kg CFC- } \\
11 \text { eq }\end{array}$ & $\begin{array}{c}\mathrm{kg} \mathrm{SO}_{2} \\
\text { eq }\end{array}$ & $\begin{array}{c}\mathrm{kg} \mathrm{PO}_{4} \\
\text { eq }\end{array}$ & $\begin{array}{c}\mathrm{kg} \mathrm{C}_{2} \mathrm{H}_{4} \\
\text { eq }\end{array}$ & MJ & MJ & & & $\mathrm{m}^{3}$ & $\mathrm{~kg}$ & kg \\
\hline $\begin{array}{c}\mathrm{kg} \mathrm{CO}_{2} \\
\text { eq }\end{array}$ & $\begin{array}{c}\text { kg CFC- } \\
11 \text { eq }\end{array}$ & $\begin{array}{c}\mathrm{kg} \mathrm{SO}_{2} \\
\mathrm{eq}\end{array}$ & $\begin{array}{c}\mathrm{kg} \mathrm{PO}_{4} \\
\text { eq }\end{array}$ & $\begin{array}{c}\mathrm{kg} \mathrm{C}_{2} \mathrm{H}_{4} \\
\text { eq }\end{array}$ & MJ & MJ & MJ & MJ & $\mathrm{m}^{3}$ & $\mathrm{~kg}$ & $\mathrm{~kg}$ \\
\hline $\begin{array}{c}\mathrm{kg} \mathrm{CO}_{2} \\
\text { eq }\end{array}$ & & & & & & & & & & & \\
\hline
\end{tabular}

Table 37: Characterization Factors in Cladding EPDs

\subsubsection{Cut-Off Rules}

Cut-off rules eliminated more Cladding and Flooring EPDs than Insulation EPDs, as many of the PCRs had different cut-off rule requirements (required to be identical for comparability as per ISO 14025). However, there is a major problem with using cut-off rules to access two EPDs' comparability: it's not always known whether or not the cut-off rules are applied. EPDs cannot be compared if they have two different sets of cut-off rules, but if both EPDs do not have the cut-off rules applied as part of their LCA, they should be comparable. Under the current governing standards and guidelines for EPDs, stating whether the cut-off rules were applied is not mandatory or suggested, and very few of the EPDs analyzed disclosed whether cut-off rules 
were applied. Theoretically, the percentage of comparisons in each of the product categories should rise, especially in categories where small percentages of material, mass and energy are not used, and where hazardous materials are not part of the product's life cycle.

\subsubsection{Mandatory Content for Comparison}

The mandatory inclusion of content for comparison eliminated some comparisons in two of the product categories. In the Insulation category, the non-comparisons came from one EPD not declaring the date of publication, which in turn blurs the period of validity. In the Cladding category, the non-comparisons came from documents that were labelled as "Environmental Product Declarations", but did not follow or mention ISO 14025. These documents did not meet the criteria in this section such as PCR identification, period of validity, or information on which life cycle stages are not considered. In fact, the products would reference ISO 14021, a standard for self-declared environmental claims, which means they are not EPDs.

\subsubsection{Comparison of Non-Critical Content Requiring Caution}

There are eight elements of Non-Critical Content Requiring Caution in the EPD Comparison Matrix. The extent of their comparability is summarized in Figure 7 and will be discussed in the following sections. 


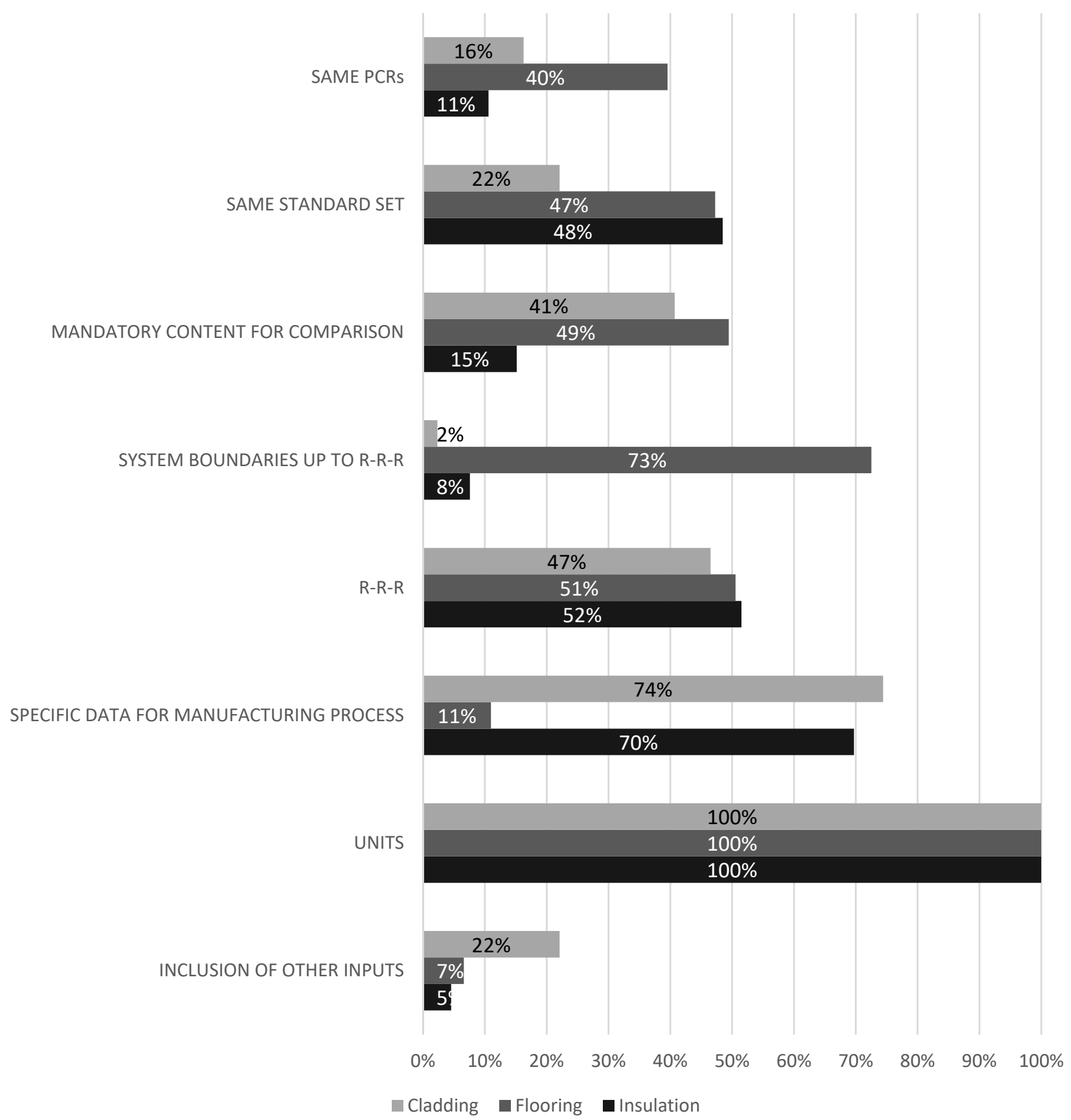

Figure 7: EPD Comparison Matrix Non-Critical Content Requiring Caution Results

\subsubsection{Same PCRs}

The Flooring product category seems to have a greater level of consistency than Cladding and Insulation in terms of the PCRs used. This can be attributed to the anecdotal use of sustainability claims in the flooring market; it was one of the early adopters of environmental product claims based on the fact that the products were installed indoors and had significant 
effects on human health (VOCs). Cladding products used 5 different PCRs, Flooring used 3 different PCRs, and Cladding used 5 different PCRs.

\subsubsection{Same Standard Set}

The Same Standard Set category is not expected to be $100 \%$, as there will be some that cannot be compared based on the fact that EN 15804 is a European-only standard, and will not apply to EPDs from North America. However, the deficiency in the Cladding section can be attributed to one of two factors. First, more EPDs did not follow EN 15804 in the Cladding category than the two others. Comparisons in the Cladding column both followed EN $1580422 \%$ of the time, compared to $47 \%$ for Flooring and $48 \%$ for Insulation. Secondly, some "EPDs" in the Cladding category did not follow ISO 14025 (marketed as EPDs, but not actually EPDs, explained previously in Section 4.3.1).

\subsubsection{Mandatory Content for Comparison}

The discrepancy in the Mandatory Content for Comparison section for Insulation is due to many of the EPDs in the product category not including Additional Environmental Information in the declaration, which was true for half of the EPDs. The other two product categories had more scattered exclusions compared to that of Insulation but had a greater number of EPDs including all mandatory content.

\subsubsection{System Boundaries, Reuse-Recovery-Recycling}

The consistency in the Flooring product category can be seen again in the System Boundaries up to Reuse-Recovery-Recycling Potential section, as the EPDs were generally consistent in declaring the same life cycle stages in a full cradle-to-grave scope. Products in the Insulation and Cladding categories did not do this as frequently as those in Flooring. However, when taking the use of the Reuse-Recovery-Recycling Potential stage into account, all sections were at approximately the same level of consistency.

\subsubsection{Specific Data for Manufacturing}

Even with Flooring being the most consistent category in earlier categories, it is substantially lower in the Specific Data for Manufacturing section. This is due to half of the EPDs (7) in this section not mentioning whether the manufacturing is specific or generic, and two used specific data. Even in the Insulation and Cladding categories, many of the deficiencies within this section arise from the declaration either not stating or being unclear about the use of specific data for the manufacturing process. 
6.3.2.6. Units

All EPDs in every product category used the same units in their declarations (SI). There were rare instances where an EPD would be written using SI units but declare a certain impact category in an IP unit, such as gallons in Freshwater, which is easily convertible.

\subsubsection{Other Inputs}

In regards to the Inclusions of Other Inputs which include the period of validity, reference service life, LCA methodology, mentions of comparability with other EPDs, there is no correlation to reason why this section is low for all three product categories. However, the Flooring category is the most consistent of the three categories, even though it does not have the highest percentage for comparison: all EPDs declared the period of validity and the reference service life, which the other categories did not do.

\subsubsection{Summary of Results}

This section is a summarization of the results from the Scoring portion of the EPD Comparison Matrix. This will cover the amount of comparisons that were deemed successful. Additionally, this section will cover the number of comparisons that had the same PCR, and within that, the number that would be theoretically not comparable, albeit having the same PCR.

It is noteworthy that while the same PCR is intended to allow comparison of EPDs, this was not always the case; conversely, several EPDs based on different PCRs were, in fact, comparable.

Table 39 summarizes the results from the EPD Comparison Matrix. The table outlines the total number of comparisons completed for each product category and quantifies which comparisons were valid and invalid. The comparisons are split into two different categories: comparisons that were valid and had same/different PCRs, and comparisons that were invalid with same/different PCRs.

\begin{tabular}{|c|c|c|c|c|}
\hline \multirow{2}{*}{\multicolumn{2}{|c|}{ Comparisons }} & \multicolumn{3}{|c|}{ Product Category } \\
\hline & & Insulation & Flooring & Cladding \\
\hline \multicolumn{2}{|l|}{ Total Comparisons } & 66 & 91 & 91 \\
\hline \multirow[b]{2}{*}{ Valid Comparisons } & Same PCRs & $4(6 \%)$ & $20(22 \%)$ & $0(0 \%)$ \\
\hline & $\begin{array}{l}\text { Different } \\
\text { PCRs }\end{array}$ & $3(5 \%)$ & $0(0 \%)$ & $\mathbf{0}(0 \%)$ \\
\hline \multirow{2}{*}{ Invalid Comparisons } & Same PCRs & $2(3 \%)$ & $16(18 \%)$ & $14(15 \%)$ \\
\hline & $\begin{array}{l}\text { Different } \\
\text { PCRs }\end{array}$ & $57(86 \%)$ & $55(60 \%)$ & 77 (85\%) \\
\hline
\end{tabular}

Table 38: EPD Comparison Matrix Results 
There are both expected and unexpected results arising from the comparison of EPDs in the Comparison Matrix. Stated broadly, the diversity of underlying PCRs (both valid and invalid) is not surprising, especially in the Insulation and Cladding categories where there are a greater number of PCRs used (five) as opposed to the Flooring category (three). However, it is not known whether the lower amount of PCRs available in the Flooring category is due to a greater level of harmonization within the product category, a lack of development for new PCRs, or by chance from the EPDs sourced for the comparison exercise.

The large proportion of invalid comparisons from two EPDs with different PCRs (the last row in Table 37) is an expected result. EPDs with different PCRs are deemed incomparable by ISO 14025 , and the reasoning for this becomes evident through the comparison process. When one or both of the EPDs being compared are either missing information or declare components in the EPD that much be identical for comparison in different ways, comparison is not permissible.

The number of valid comparisons with different PCRs (the second data row in Table 37) is somewhat surprising. Due to harmonization efforts in the industry, especially with standards such as EN 15804 (the purpose of which is to aid comparison by acting as a "core PCR"), it was hypothesized that there would be a greater level of comparison within this specific category. However, only one product category (Insulation) exhibited valid comparisons from different PCRs. All EPDs that were compared and fell into this category all followed EN 15804. By contrast, $48 \%$ of comparisons in Insulation followed EN 15804, 47\% in Flooring, and 22\% in Cladding. According to this metric, there possibly should have been more comparison in the Insulation category, possibly an equal amount of comparison in the Flooring category, and in the Cladding category to a lesser extent.

An unexpected result from this exercise is the relationship between valid and invalid comparisons between EPDs with the same PCR. The fact that there are invalid comparisons from two EPDs with the same PCR is concerning, as ISO 14025 permits such comparison and is written to enable this.

In the Cladding category, comparisons that had the same PCR but were deemed invalid was due to two crucial categories within the EPD Comparison Matrix (described in Section 4.3.1): Impact Category Characterization Factors (for all comparisons), or Cut-Off Rules (only for a minority of comparisons). In the Impact Category Characterization Factors section, some EPDs did not declare some of the key impact categories identified or declared them with a characterization factor that could not be "converted" (discussed in detail and defined in Section 
4.1.4 and 4.3.1): global warming potential, ozone depletion, acidification, eutrophication, formation of tropospheric ozone, non-renewable energy use, renewable energy use, nonrenewable resource use, renewable resource use, consumption of freshwater, hazardous waste, and non-hazardous waste.

The Crucial Categories that eliminated comparison between two EPDs with the same PCR can be seen in Table 40 below. The number in each cell represents the amount of invalid comparisons that failed each specific crucial comparison category. Comparisons may have been eliminated from non-compliance with multiple categories.

\section{Crucial Categories}

Total Invalid Comparisons with Same PCR

Same Functional/Declared Unit
System Boundaries Separated or
Same
Impact Category Characterization
Factors
Mandatory Content for Comparison
Cut-Off Rules

\section{Product Category}

\begin{tabular}{l|ll} 
Insulation $\quad$ Flooring $\quad$ Cladding
\end{tabular}

Table 39: EPDs with Same PCR, Invalid Comparison Results

The reason for this lack of alignment of EPDs using the same PCR is unknown, although there are some possibilities that have been identified through this process. Firstly, it is a possibility that the PCR is not of a sufficient level of quality. It could be that the PCR is not written stringently enough, or does not mandate specific characteristics that would render comparison possible. Secondly, the EPD may not be written to the level of quality it must be, or the LCA process behind the EPD may be conducted in a way that does not follow the PCR. This leads to discrepancies in the text of the EPD or the data from the LCA between EPDs with the same PCR. Thirdly, the verification process may not be stringent enough to identify problems within EPDs. At the time of publication, two EPDs written to the same PCR should be comparable, and the verification process should be an avenue to identify deficiencies that can invalidate comparison. Finally, this could be due to a combination of the above characteristics identified. 


\section{Using the Product Category Rule Comparison Matrix}

This section describes the process of evaluating whether two PCRs are comparable in the identified product categories related to construction projects described in Section 5.1. Outlined the method used to complete the PCR Comparison Matrix and the key results and discussion arising from the completion of the matrix.

\subsection{Comparison Methodology}

The PCR Comparison Matrix was completed using the chart as described in Section 5. The chart was completed row by row, by choosing a PCR and filling in all required information along the row of the Inputs section, rather than column by column.

PCRs analyzed in the matrix for each product category were generated from the EPD Comparison Matrix. All PCRs used by the selected EPDs were analyzed in the PCR Comparison Matrix. PCRs that are either expired or have been replaced that were present in the EPD Comparison Matrix were also considered for comparison. In total, five PCRs were compared in the Insulation product category, three PCRs in the Flooring category, and five PCRs in the Cladding category. Some overlap exists where the same PCR was used for two different product categories.

All information within the EPD Comparison Matrix Inputs section was sourced directly from the PCR.

The Comparison section was completed using results from the Inputs section. Similar to the EPD Comparison Matrix, factorial comparisons were constructed in order to compare every PCR within the matrix to every other PCR (i.e. $A$ vs $B, A$ vs $C, A$ vs $D, B$ vs $C, B$ vs $D, C$ vs $D$ ). Either a "yes" or "no" was inserted for each factorial comparison based on the information within the PCR. Some sections require a "yes" or "no" based on both PCRs both including information. An example of this is in the System Boundaries category, where the two PCRs being compared are required to declare the same system boundary (both declare Transport to Landfill, "yes"). Other scenarios are more precise such as in the Data Quality Requirements section, where both PCRs being compared have to use specific data in order to get a "yes".

The Summarization section, and therefore the Scoring section is automatically calculated when the "yes" and "no" cells are completed within the Comparison section. PCRs that can be compared can then be seen at the culmination of the Scoring section. 


\subsection{Results and Discussion}

\subsubsection{Comparison of Crucial Content}

There are seven elements of Crucial Content in the PCR Comparison Matrix. The extent of their comparability is summarized in Figure 8 below and will be discussed in the following sections.

PRODUCT CATEGORY DEFINIITION AND DESCRIPTION

FUNCTIONAL UNIT SYSTEM BOUNDARY

$$
\begin{aligned}
& \text { CUT-OFF RULES } \\
& \text { DATA QUALITY } \\
& \text { ALLOCATION RULES } \\
& \text { INVENTORY INDICATORS, } \\
& \text { IMPACT CATEGORIES, } \\
& \text { LCIA CHARACTERIZATION } \\
& \text { FACTORS, LCIA METHODS }
\end{aligned}
$$

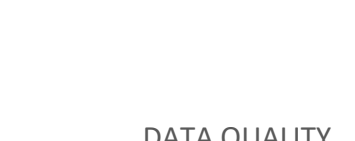

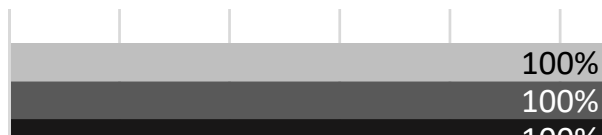

$100 \%$

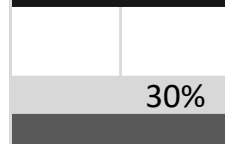

$30 \%$

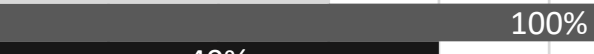

$40 \%$

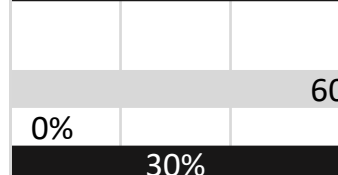

$30 \%$

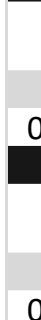

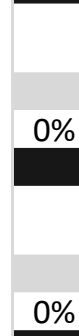

$30 \%$

$$
10 \%
$$
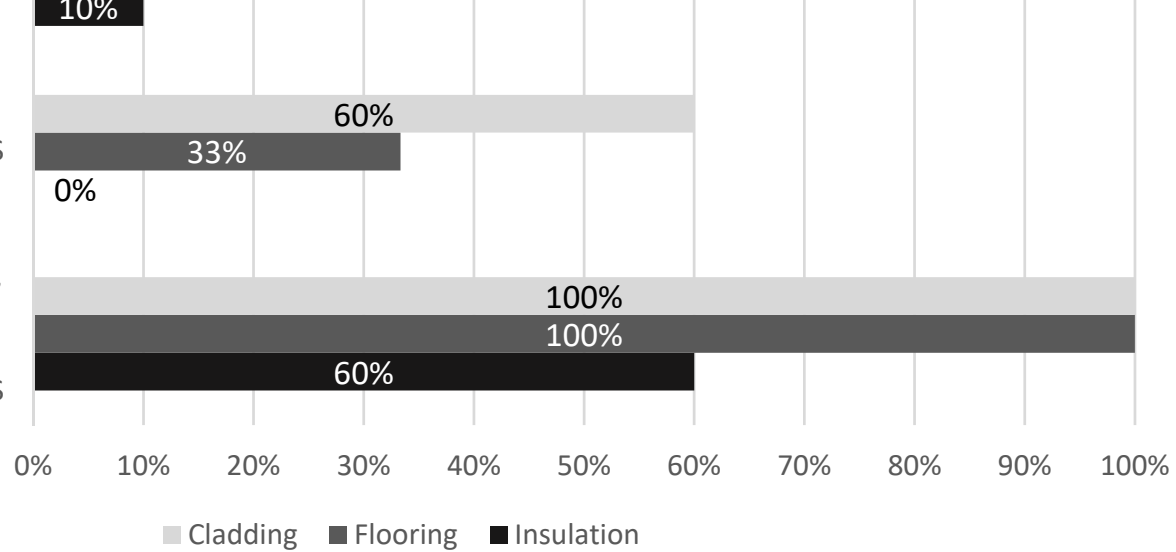

Figure 8: PCR Comparison Matrix Crucial Comparison Results

\subsubsection{Product Category Definition and Description}

The PCRs compared were consistent in the product category definition and description, which outlines what products should use the PCR for developing their EPD. PCRs were either 
targeted directly to the product categories identified in this thesis (cladding, flooring, or insulation) or were targeted to all construction products and services.

\subsubsection{Functional Unit}

One of the major deficiencies in the functional unit came from PCRs targeted at all construction products and services being intentionally vague in the definition of the declared unit. These PCRs cannot be specific in what they mandate to be used as a declared unit because of the wide range of products the PCR accommodates. In these cases, the PCR defines the declared unit as mass (i.e. $1 \mathrm{~kg}$ ), volume $\left(1 \mathrm{~m}^{3}\right)$, area $\left(1 \mathrm{~m}^{2}\right)$, length $(1 \mathrm{~m})$, or an item (1 brick). This is dissimilar from PCRs written for a specific product category, such as flooring, where all PCRs use $1 \mathrm{~m}^{2}$ of flooring material for the functional unit.

Insulation had a special case with some of the PCRs. There were two of the five EPDs analyzed that stated the declared unit as either: $1 \mathrm{~m}^{2}$ of insulation material with a thickness that gives a design thermal resistance of $1 \mathrm{~m}^{2} \cdot \mathrm{K} / \mathrm{W}$ or $1 \mathrm{~m}^{3}$ of insulation material. These PCRs are not comparable with other PCRs written specifically for insulation material that state the functional unit as the $1 \mathrm{~m}^{2}$ option. This is because in some scenarios, one EPD uses $1 \mathrm{~m}^{2}$ and the other uses $1 \mathrm{~m}^{3}$ and neither provide a ratio or factor to convert the results to be comparable to one another.

\subsubsection{System Boundaries}

This section requires the description of the system boundaries to be exact. For example, in the Raw Material Acquisition stage, the processes that can be included are extraction and processing of raw materials (e.g. mining processes), biomass production and processing, reuse of products or materials from a previous product system, and generation of electricity, steam and heat from primary energy resources, including extraction, refining and transport thereof. Two PCRs need to have the exact same system boundary for each stage, as they are required to be identical. A PCR missing only one of these processes would be excluded from the comparison, which would explain the deficiency across the product categories in this section. An example of two system boundaries that are not comparable is illustrated in Table 41. 
PCR A (Raw Material Acquisition Stage)

Extraction and processing of raw materials, biomass production and processing and recycling processes of secondary materials from a previous product system, but not including those processes that are part of the waste processing in the previous product system, referring to the polluter pays principle Generation of electricity, steam and heat from primary energy resources, also including their extraction, refining and transport. This also includes energy needed for raw material supply and energy for manufacturing in core process

Energy recovery and other recovery processes from secondary fuels, but not including those processes that are part of waste processing in the previous product system

Processing up to the end-of-waste state or disposal of final residues including any packaging not leaving the factory gate with the product
PCR B (Raw Material Acquisition Stage)

Extraction/Production of raw materials
Average transport of raw materials from extraction/production to manufacturer

\section{Table 40: PCR System Boundary Comparison}

The two system boundaries for PCR A and PCR B are very similar but not exactly identical, which is one example of an invalid comparison. For example, PCR A includes disposal of residues and packaging not leaving the factory gate, where PCR B does not.

\subsubsection{4. $\quad$ Cut-Off Rules}

Similar to the System Boundaries section, cut-off rules must be exactly identical for comparison. A PCR with cut-off rules that exclude $1 \%$ of mass and $1 \%$ of energy is not comparable to a PCR that excludes $2 \%$ of mass and $2 \%$ of energy. Small differences in the cut-off rules across all PCRs affected comparison in every product category.

\subsubsection{Data Quality}

Data Quality focuses on the temporal, geographical, and technological requirements of the data used in the LCA. Similar to the previous sections described, small differences in the requirements for any of the three requirement categories caused the deficiencies in comparison. 
Two temporal examples taken directly from the text of two Insulation PCRs that were not deemed identical include: "Data sets used for calculation shall have been updates within the last 10 years for generic data, last 5 years for specific data. Data sets shall be based on 1 year averaged data", and "Specific data no older than 3 years. Generic data no older than 10", where the main difference is the stipulation of the age of specific data.

Differences of this type can be seen for both the geographical and technological requirements of the data used in the LCA as stipulated in the PCR.

\subsubsection{Allocation Rules}

Similar to the previous sections, small differences in the allocation rules, such as specific rules based on combined multi-outputs (i.e. one raw material acquisition process producing multiple materials) can cause deficiencies in comparison. Two examples of excerpts from allocation rules in PCRs is illustrated in Table 42.

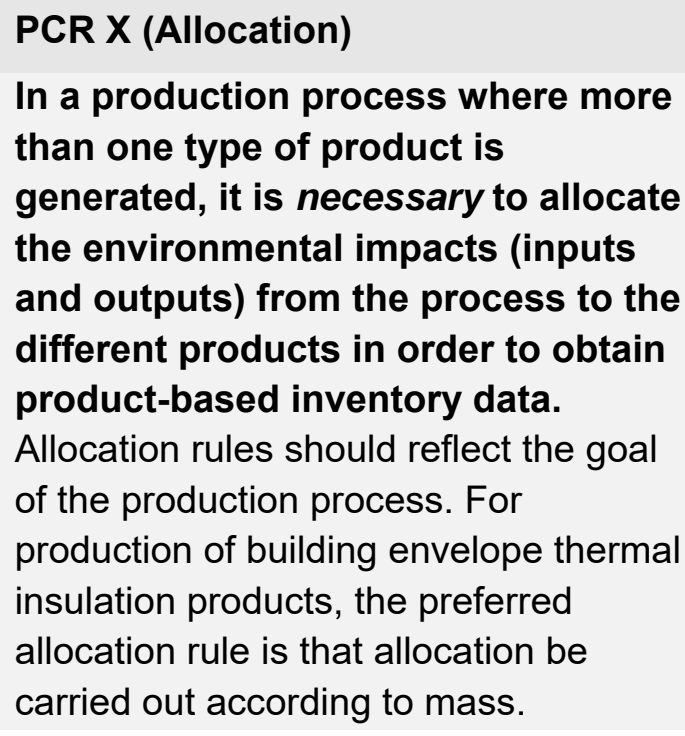

\section{PCR Y (Allocation)}

Most industrial processes produce more than the intended product. Normally more than one input flow is needed to produce one product and sometimes products are co-produced with other products. As a rule, the material flows between them are not distributed in a simple way. Intermediate and discarded products can be recycled to become inputs for other processes. When dealing with systems involving multiple products and recycling processes, allocation should be avoided as far as possible. Where unavoidable, allocation should be considered carefully and should be justified.

\section{Table 41: PCR Allocation Rules Comparison}

In this example, the PCRs do not share the exact same allocation rules (see bold text), and therefore are incomparable. This is only one cursory example of the allocation rules that are published in a PCR (allocation sections are multiple pages in length), and to analyze them in this excerpt fashion is not advisable, Table 42 is only intended for illustrative purposes. There multiple other scenarios where the allocation rules do not match in PCR X and PCR Y, most of which is due to PCR X including information where PCR Y does not, or vice versa. 


\subsubsection{7. $\quad$ Other Inputs}

The Other Inputs section, which includes inventory indicators, impact categories, LCIA characterization factors, and LCIA methods, was consistent in the Flooring and Cladding categories. The non-comparisons present in the Insulation category are due to a PCR requiring that photochemical ozone creation is stated in $\mathrm{kg} \mathrm{C}_{2} \mathrm{H}_{2}$ eq (ethyne), rather than $\mathrm{kg} \mathrm{C}_{2} \mathrm{H}_{4}$ eq (ethene).

It should be noted that a few of the PCRs that were analyzed included a set of characterization factors for North America ( $\mathrm{mol} \mathrm{H}+$ eq, $\mathrm{kg} \mathrm{N} \mathrm{eq,} \mathrm{kg} \mathrm{O}_{3} \mathrm{eq}$ ), and a set for Europe ( $\mathrm{kg} \mathrm{SO}_{2}$ eq, $\mathrm{kg} \mathrm{N}$ eq, $\mathrm{kg} \mathrm{C}_{2} \mathrm{H}_{4} \mathrm{eq}$ ), and it is up to the publisher of the EPD to pick which to use. These PCRs were not marked as incomparable to a PCR that would only include a "European" set of characterization factors.

\subsubsection{Comparison of Non-Critical Content Requiring Caution}

There are eight elements of Non-Critical Content Requiring Caution in the PCR Comparison Matrix. The extent of their comparability is summarized in Figure 9 below and will be discussed in the following sections.

\subsubsection{Manufacturing Data Both Specific}

The deficiencies in this section were a result of the PCR not stating any specific or generic requirements for the manufacturing data in the LCA (present in the Insulation product category). Another possibility of a deficiency in this category would be a PCR not requiring the use of specific data for manufacturing processes. This was seen in the EPD Comparison Matrix, where declarations used generic data for the manufacturing stage, but was not present in any of the PCRs analyzed.

\subsubsection{2. $\quad$ Adherence to Same Standards}

All PCRs analyzed in the Cladding category followed both ISO 14025 and EN 15804. The noncomparisons in the Flooring and Insulation categories arise from PCRs that follow ISO 14025 but do not follow EN 15804.

\subsubsection{Same LCA Type}

This category is consistent across all product categories, all PCRs mandate that the LCA must be attributional, and not consequential. 
MANUFACURING DATA

BOTH SPECIFIC

ADHERANCE TO SAME STANDARDS

LCA TYPE SAME?

DECLARATION OF MATERIALS AND SUBSTANCES THAT

AFFECT HUMAN HEALTH/ENVIRONMENT

ADDITIONAL INFORMATION REQUIREMENTS

UNITS

DATA SOURCES

METHODS OF DATA COLLECTION
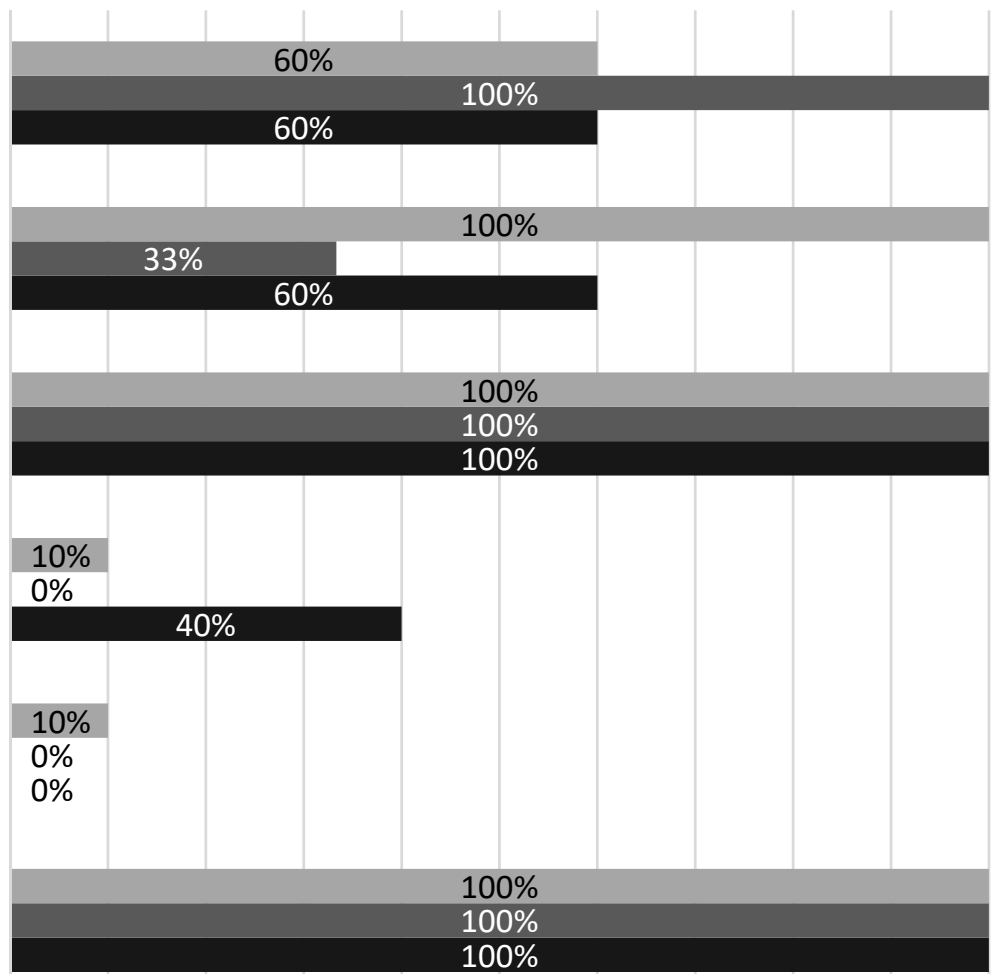

$60 \%$

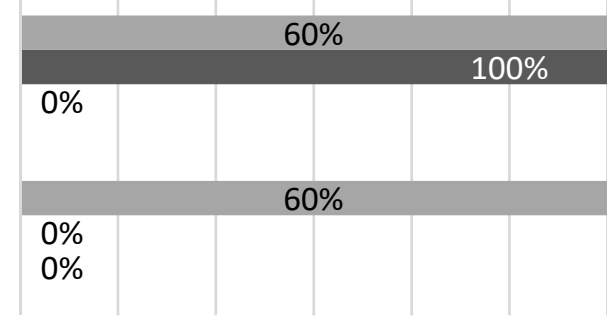

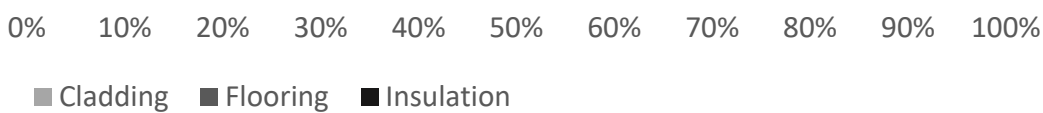

Figure 9: PCR Comparison Matrix Non-Critical Content Requiring Caution Comparison Results

\subsubsection{Hazardous Substances}

Hazardous substances that must be declared in the EPD were inconsistent across all product categories. It should be noted that provisions for declaring hazardous substances were included at an appropriate level, but the exact provisions and wording was inconsistent from PCR to PCR.

\subsubsection{Additional Information Requirements}

Additional information requirements related to environmental effects was inconsistent across all product categories. Similar to the hazardous substances section, it should be noted that 
provisions for declaring additional environmental information were included at an appropriate level, but the exact provisions and wording was inconsistent from PCR to PCR.

\subsubsection{Units}

All PCRs required the same system of measurement $(\mathrm{SI})$ across all product categories.

\subsubsection{Data Sources}

The Data Sources section focuses on a hierarchal order of generic data sources or the criteria that can be used to determine whether a generic data source is appropriate. Equivalency in this category was achieved through the PCR's reference to CEN/TE 15941 - Sustainability of construction works - Environmental product declarations - Methodology for the selection and use of generic data. Most incomparable scenarios arose when PCRs either did not include a discussion of generic data sources or failed to mention CEN/TE 15941 for the use of generic data.

\subsubsection{Methods of Data Collection}

Methods of data collection focus the requirements or suggested means of primary data collection. All of the inconsistencies in this section across all product categories was a result of the PCRs not including specific provisions regarding primary data collection.

\subsubsection{Summary of Results}

Table 43 below summarizes the results from the PCR Comparison Matrix. The table outlines the total number of comparisons completed for each product category and quantifies which comparisons were valid and invalid. None of the PCR comparisons completed were valid due to a combination of failures within the Crucial Content section, discussed in Section 8.2.1. In the Insulation and Cladding categories, most non-comparisons were due to deficiencies in all sections except the product category definition and the characterization factor sections. In the Flooring category, the non-comparisons were due to small differences in the system boundaries, cut-off rules, data quality requirements, and allocation rules of the PCRs. 


\begin{tabular}{|c|c|c|c|}
\hline \multirow[b]{2}{*}{ Comparisons } & \multicolumn{3}{|c|}{ Product Category } \\
\hline & Insulation & Flooring & Cladding \\
\hline Total Comparisons & 10 & 3 & 10 \\
\hline Valid Comparisons & $\mathbf{0}(0 \%)$ & $\mathbf{0}(0 \%)$ & $\mathbf{0}(0 \%)$ \\
\hline Invalid Comparisons & $10(100 \%)$ & $3(100 \%)$ & $10(100 \%)$ \\
\hline \multicolumn{4}{|c|}{ Table 42: PCR Comparison Matrix Results } \\
\hline \multicolumn{4}{|c|}{$\begin{array}{l}\text { The results of all PCRs in each product category not being comparable to one another is very } \\
\text { unexpected. It was hypothesized that there would be some incomparability, but at least some if } \\
\text { not all PCRs that were based on EN } 15804 \text { would be comparable. }\end{array}$} \\
\hline \multicolumn{4}{|c|}{$\begin{array}{l}\text { The Crucial Categories that eliminated comparison between PCRs can be seen in Table } 44 \\
\text { below. The number in each cell represents the amount of comparisons that failed each specific } \\
\text { crucial comparison category. Comparisons may have been eliminated from non-compliance with } \\
\text { multiple categories. }\end{array}$} \\
\hline
\end{tabular}

Product Category

\section{Crucial Categories}

\begin{tabular}{l|ll} 
Insulation & Flooring $\quad$ Cladding
\end{tabular}

Total Invalid Comparisons

\section{0}

Product Category Definition and

Description

Functional/Declared Unit

System Boundaries

Data Quality Requirements

Cut-Off Rules

Allocation Rules

Life Cycle Impact Assessment

Requirements

\begin{tabular}{ccc}
$\mathbf{0}(0 \%)$ & $\mathbf{0}(0 \%)$ & $\mathbf{0}(0 \%)$ \\
\hline $\mathbf{6}(60 \%)$ & $\mathbf{0}(0 \%)$ & $\mathbf{7}(70 \%)$ \\
$\mathbf{6}(60 \%)$ & $\mathbf{3}(100 \%)$ & $\mathbf{4}(40 \%)$ \\
\hline $\mathbf{9}(90 \%)$ & $\mathbf{3}(100 \%)$ & $\mathbf{4}(40 \%)$ \\
$\mathbf{7}(70 \%)$ & $\mathbf{3}(100 \%)$ & $\mathbf{4}(40 \%)$ \\
$\mathbf{1 0}(100 \%)$ & $\mathbf{1}(33 \%)$ & $\mathbf{4}(40 \%)$ \\
$\mathbf{4}(40 \%)$ & $\mathbf{0}(0 \%)$ & $\mathbf{0}(0 \%)$
\end{tabular}

Table 43: PCR Invalid Comparison Results

It seems the only category that is consistently comparable is the product category definition and description category. The results for crucial category incomparability are scattered, and there isn't a dominant reason that can be easily identified as to why this is the case. However, this 
incomparability raises a few questions: would the comparison of PCRs be suggested at this time? Is there not enough consistency or harmonization between PCRs to compare them in a meaningful way? From the results of this study, it seems that comparison between EPDs is more relevant in the current climate of declarations than comparing the PCRs behind them. As harmonization efforts increase in the future, comparing PCRs may be viable for establishing large-scale comparability schemes within product databases.

A Simplified EPD Comparison and Simplified PCR Comparison were initially intended to be developed in the following section, Development of a Simplified Comparison Template. However, due to the results of the PCR Comparison Matrix, it would appear that there is no need for a simplified comparison method for PCRs if comparability does not exist. 


\section{Development of a Simplified Comparison Template}

Based on lessons learned from the application of the EPD and PCR Comparison Matrices discussed previously, a simplified approach has been developed to facilitate a more streamlined comparison approach. The Simplified EPD Comparison Template developed and the rationale for not developing a simplified PCR comparison will be discussed in the following sections.

\subsection{Simplified EPD Comparison Template}

The Simplified EPD Comparison Matrix was built by questioning which sections of the original EPD Comparison Matrix were appropriate for determining whether two EPDs could be compared or not in a building project context. Ideally, this Simplified Comparison would be used as a "first-pass" tool: a practitioner could use the Simplified Template in lieu of the full Comparison Template to determine comparability and aid the comparison process, and use the full Comparison Template for a deeper comparison if desired or required.

The Simplified EPD Comparison Template has three major alterations from the EPD Comparison Matrix:

1. Reduction of Input and Comparison cells to be filled from 64 to 49 (refer to Table 45);

2. Comparison cells which are required to be filled have been adjusted from all manual to only three manual cells, and;

3. Comparison is now tiered: the most important information for comparison is in the chart first. Therefore, incomparable EPDs can be identified after i.e. 6 cells instead of 64 . This is demonstrated in Figure 10.

These measures are intended to both simplify and expedite comparison for designers using EPDs as decision making tools. 


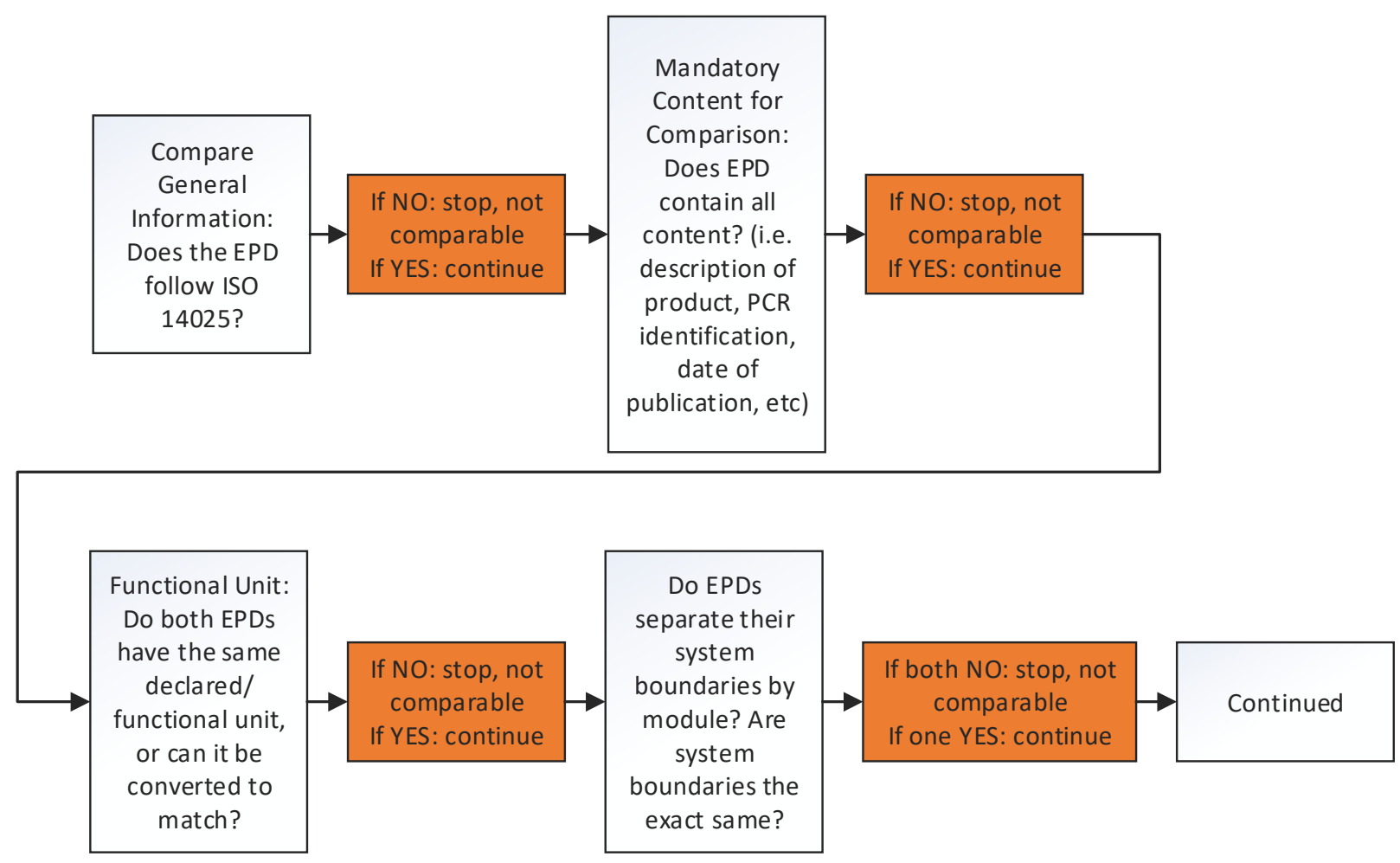

Figure 10: Simplified EPD Comparison Template: Tiered Comparison Demonstration

It is organized in the same manner as the original EPD Comparison Template with the categories (referring to alteration 1 above) omitted based on an evaluation using three simple criteria: information was deemed not necessary for the comparison; information that was deemed superfluous for the comparison, and; or information that was deemed too complex for its minimal effect on the comparison.

Table 45 outlines which of the major components were eliminated from the EPD Comparison Matrix to create the Template, and the justification or reasoning behind each elimination. 


\section{Component Removed}

Adherence to ISO 21930 (EN 15804 modified)

\section{Justification/Reasoning}

Deemed superfluous as it is not required by any standard. EN 15804 has been modified to not have any effect on comparison results, as it may still have importance in EPD comparison.

All mandatory content that must be included, but is not crucial for comparison

- Identification and Description of Organization

- Product ID (model number)

- Name and address of program operator

Not necessary for comparison.

- Additional environmental information

- Information on where explanatory information may be obtained

- Statement that EPDs from different program operator's may not be comparable

Data Quality Requirements, except for the Manufacturing/Production Stage

Too complex of a process for minimal effect on comparison.

Cut-Off Rules

Too complex of a process for minimal effect on comparison.

Units

Superfluous (was not a factor in the EPD Comparison Matrix results).

Period of Validity

Superfluous.

Superfluous. It is still included in the template,

Service Life Time Period as it has importance for EPDs with annualized impacts. It is not a column that is required to be filled.

LCA Methodology

Not necessary for comparison.

Mentions of Comparability

\section{Table 44: Omissions from Simplified EPD Comparison Template}

There are two possible ways that the Simplified EPD Template can be used. The first way to use this Template is the manual version, which only includes the Inputs section. This is a much simpler version that provides the user with the ability to compare EPDs manually by filling out the section in a fashion as they so desire. The second way to use this Template is the automatic version. This version of the Simplified EPD Comparison Template is similar to the original EPD Comparison Matrix in that it has all the same categories: Inputs, Comparison, Summarization, 
and Impact Category Results. However, all cells in the latter three sections are fully automated where it is possible, making the tool more user-friendly than the full Comparison Matrix.

In addition to this, there are instructions and explanatory information for each of the columns within the Inputs and Comparison sections in both versions of the Template. An excerpt from the Simplified EPD Comparison Template showing this is presented in Table 46 below.

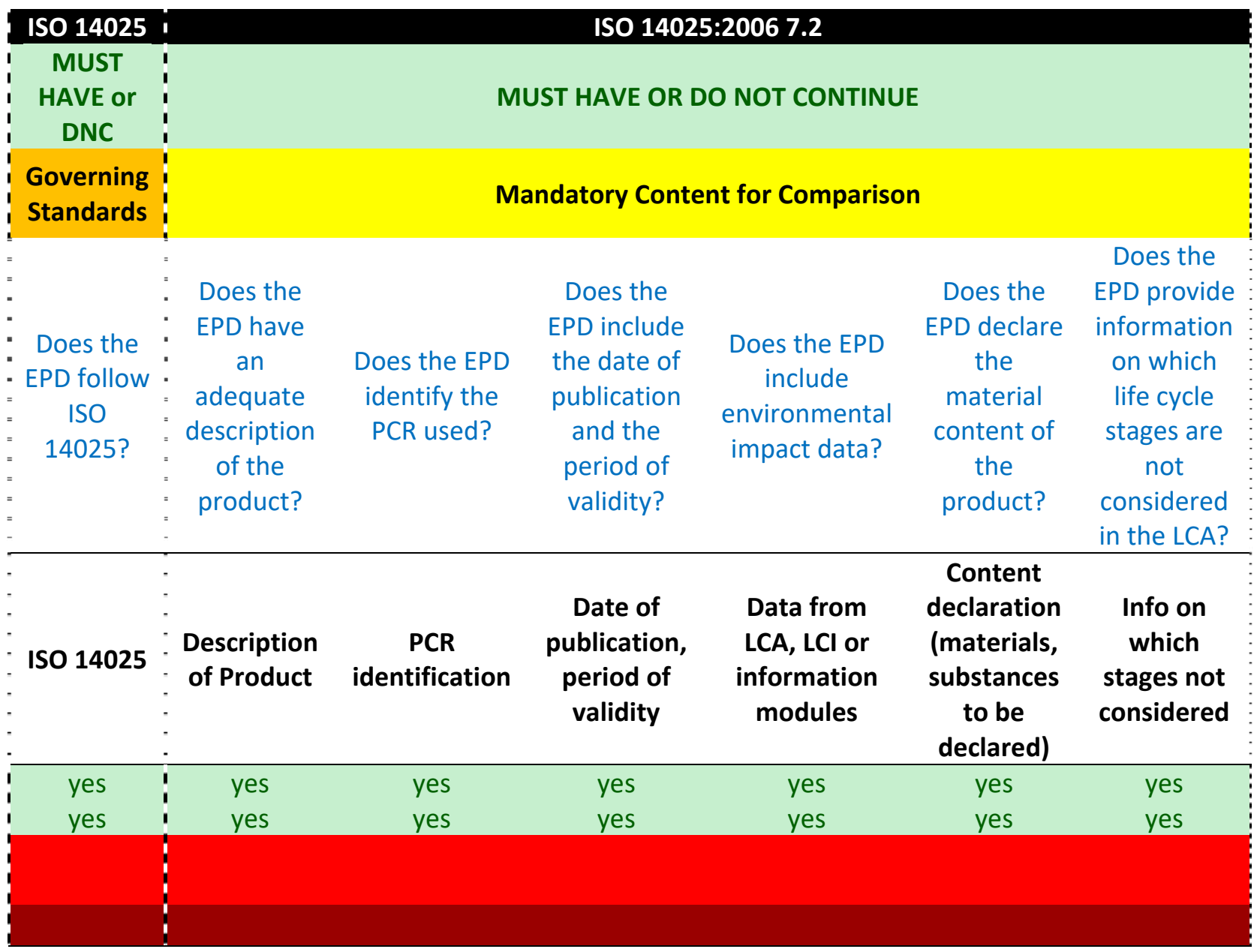

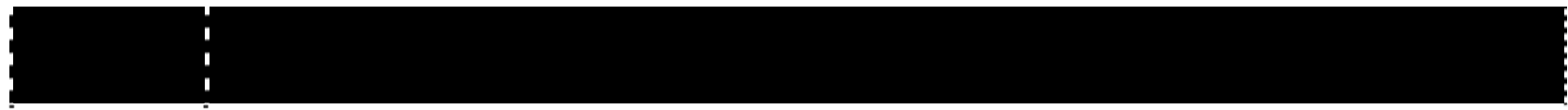

Do both

EPDs : Do both EPDs include the each of the respective mandatory content items for follow ISO 14025 ?

! yes : yes yes yes yes yes

Table 45: Simplified EPD Comparison Template Excerpt

The columns that remain in the Simplified EPD Comparison Template from the EPD Comparison Matrix are described below. The first grouping of columns are columns that the 
EPD must include. Essentially, these columns were identified as important enough to preclude any comparison if they are not included or followed as part of the EPD. If any EPD doesn't pass these two sections, the comparison cannot continue. Each of the elements will be followed by the section in a relevant standard where the context for the column was sourced, and a full description of the column. The phrase "Adapted" is used in lieu of a referenced standard if the criterion was developed as part of this research.

1. Governing Standards (ISO 14025): Does the EPD follow ISO 14025?

2. Mandatory Content for Comparison, Crucial for Comparison (ISO 14025 7.2): Does the EPD include all of the following components: Description of product; PCR identification; date of publication and period of validity; data from LCA, $\mathrm{LCl}$, or other information modules; content declaration (materials and substances to be declared); and information on which stages are not considered/declared.

This second grouping is focused on the comparison of EPDs, rather than an evaluation of their validity in the first grouping.

1. Functional Unit (ISO 14025 6.7.1(b)): In Inputs, what is the functional/declared unit used in the EPD? In Comparison, do both EPDs have the same functional unit, or if not, is it possible the results can be extrapolated $\left(1000 \mathrm{~m}^{2} \mathrm{vs} 1 \mathrm{~m}^{2}\right)$ or converted $\left(1000 \mathrm{~m}^{2} \mathrm{vs}\right.$ $\left.1 \mathrm{ft}^{2}\right) ?$

\section{System Boundaries:}

- In Inputs, does the EPD declare the stages listed: Raw Material Acquisition, Transportation to Manufacturer, Manufacturing, Transport to Site, Construction/Installation, Use, Maintenance, Repair, Refurbishment, Operational Energy Use, Operational Water Use, De-construction, Transport to Landfill, Waste Processing, Disposal, Reuse-Recovery-Recycling Potential. In Comparison, do both EPDs declare each stage? (ISO 14025 6.7.1(b), EN 15804 6.2)

- In Inputs, is the environmental impact data from the LCA separated by, at least, module? (A1-A3, A4-A5, B, C, D as described in Section 7.3.1.2). In Comparison, do both EPDs separate their environmental impact data? (Adapted)

- Are the system boundaries for both EPDs being compared exactly the same? (This column is only completed in the Comparison section) (Adapted) 
3. Manufacturing Data Quality (EN 15804 6.3.6): In Inputs, does the EPD use specific data for the manufacturing (or foreground) processes? In Comparison, do both EPDs use specific data for their manufacturing (or foreground) processes?

\section{Impact Categories:}

- In Inputs, what is the characterization factor used in each of the following impact categories: global warming potential, ozone depletion potential, acidification, eutrophication, formation of tropospheric ozone, use of non-renewable energy, use of renewable energy, use of non-renewable resources, use of renewable resources, consumption of fresh water, hazardous waste, and non-hazardous waste. In Comparison, do both EPDs use the same characterization factor for each respective impact category, or, can the characterization factor be "converted" to match (as described in Section 4.1.4)? (ISO 21930 8.2.2)

- In Inputs, were any of the characterization factors in the EPD converted? In Comparison, did either of the EPDs being compared convert a characterization factor? (Adapted)

5. Service Life Time Period [only included for EPDs with annualized impacts] (ISO 21930 8.2.5): In Inputs, what is the service life time period of the product? In Comparison, do both EPDs use the same service life time period?

6. File Path, Notes: Miscellaneous items for the authors benefit, what is the file path of the EPD (if electronic), and are there any notes on the EPD that does not fit into any of the prior sections.

The Summarization and Impact Category Results sections work exactly like described in Sections 4.2, 4.3, and 4.4, but have accommodated the reduced content of the Simplified EPD Comparison Matrix. The full versions of both Simplified EPD Comparison Templates, manual and automatic, are available in Appendix B.

\subsection{Simplified PCR Comparison Template}

As described in Section 7.2.3, a simplified PCR Comparison Template was initially planned to be included as part of this thesis but was ultimately not developed due to the lack of comparability within construction product PCRs. However, it should be noted that comparison between PCRs may not be appropriate now, but could possibly be a good technique to assess future harmonization efforts. 


\section{Discussion and Recommendations}

This section focuses on the issues associated with both EPDs and PCRs at the current time, and their use in the construction industry as a material sourcing tool. Suggestions for the direction of future research and specific topics for further exploration are also provided.

\subsection{Issues Associated with Environmental Product Declarations}

Through the literature review, a number of general deficiencies regarding the use of EPDs were uncovered. These included the lack of availability of products with EPDs, the transparency of the "black box" LCA process, and the distance of transportation for products that carry EPDs. However, there are a number of more specific and detailed concerns regarding EPDs.

A particularly concerning aspect regarding EPDs is the fact that there were multiple occasions where two EPDs with the same underlying PCR were not comparable. In this study, $58 \%$ of comparisons (33 of 57) completed with the same PCR were found to be invalid through the EPD Comparison Matrix. Detailed results of these invalidations are presented in Tables 37 and 38 in Section 6.3.3.

There are a number of possibilities that can explain these invalidations.

1. The instructions or verbiage in the PCR is of an insufficient level to ensure that two different authors can use the same PCR and write comparable EPDs without question.

2. The execution or authoring of the EPD is not of an appropriate level of detail or completion and therefore invalidates the comparison.

3. The third-party verification of the EPD is not stringent enough to exclude or reject EPDs that would not be comparable to any other EPD written with the same PCR.

Below, Table 47 identifies the two invalid comparisons that the same EPD in the Insulation product category of the EPD Comparison Matrix and lists the reasoning behind the invalidation.

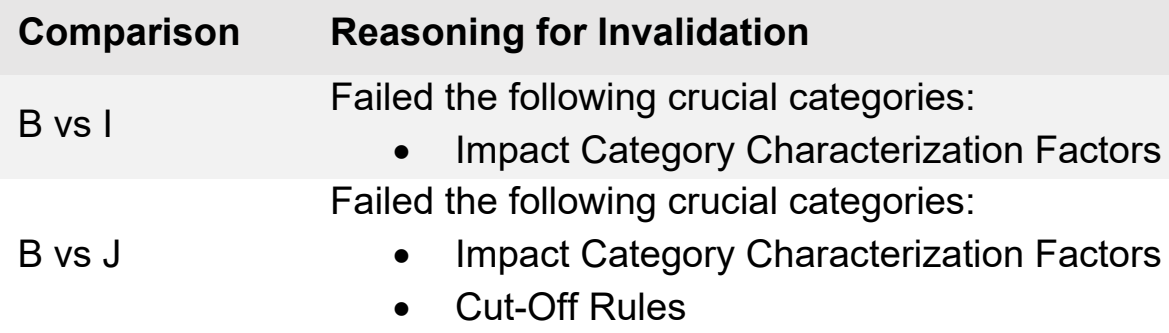

Table 46: Reasoning for Invalid Comparisons in EPD Comparison Matrix, Insulation Category 
$B$ vs $\mathrm{J}$ failed two out of five crucial categories in the EPD Comparison Matrix. The Impact Category Characterization Factors used in each EPD are shown in Table 48 below.

Impact Category
Global Warming Potential
Ozone Depletion Potential
Acidification
Eutrophication
Formation of Tropospheric Ozone
Use of Non-Renewable Energy
Use of Renewable Energy
Use of Non-Renewable Resources
Use of Renewable Resources
Consumption of Freshwater
Hazardous Waste
Non-Hazardous Waste

B

$\mathrm{kg} \mathrm{CO} 2$ eq kg CFC-11 eq $\mathrm{mol} \mathrm{H}+$ eq, converted to $\mathrm{SO}_{2}$ eq $\mathrm{kg} \mathrm{N}$ eq, converted to $\mathrm{kg}$ $\mathrm{PO}_{4}{ }^{3-}$ eq Not declared MJ MJ

MJ

MJ

gal, converted to $\mathrm{m}^{3}$

$\mathrm{kg}$

kg

\section{J}

$\mathrm{kg} \mathrm{CO} 2$ eq kg CFC-11 eq mol H+ eq, converted to $\mathrm{SO}_{2}$ eq $\mathrm{kg} \mathrm{N}$ eq, converted to $\mathrm{kg}$ $\mathrm{PO}_{4}{ }^{3-}$ eq Not declared Not declared Not declared Not declared Not declared $\mathrm{m}^{3}$

Not declared Not declared

Table 47: Impact Category Characterization Factor Invalidation Comparison, B vs J

In the above table, it is evident that the invalid comparison is due to some of the impact categories identified are not declared (shown in the bold red text).

In the PCR, there are two sections that focus on environmental impacts: one that provides the characterization factors to be employed to calculate selected environmental impacts, and another that lists a number of environmental impacts that an EPD "will" include. Table 49 shows the first section, and Table 50 shows the second. 


\begin{tabular}{|c|c|c|c|}
\hline \multicolumn{2}{|c|}{$\begin{array}{l}\text { Units for Characterization Factors of } \\
\text { Environmental Attributes for North } \\
\text { America }\end{array}$} & \multicolumn{2}{|c|}{$\begin{array}{l}\text { Units for Characterization Factors of } \\
\text { Environmental Attributes for Europe }\end{array}$} \\
\hline Impact Category & $\begin{array}{l}\text { Characterization } \\
\text { Factor }\end{array}$ & Impact Category & $\begin{array}{l}\text { Characterization } \\
\text { Factor }\end{array}$ \\
\hline $\begin{array}{l}\text { Global Warming } \\
\text { Potential }\end{array}$ & $\mathrm{kg} \mathrm{CO} \mathrm{Cq}_{2}$ & $\begin{array}{l}\text { Global Warming } \\
\text { Potential }\end{array}$ & $\mathrm{kg} \mathrm{CO} \mathrm{Cq}_{2}$ \\
\hline $\begin{array}{l}\text { Ozone Depletion } \\
\text { Potential }\end{array}$ & kg CFC-11 eq & $\begin{array}{l}\text { Ozone Depletion } \\
\text { Potential }\end{array}$ & kg CFC-11 eq \\
\hline Acidification & mol H+ eq & Acidification & $\mathrm{kg} \mathrm{SO}_{2} \mathrm{eq}$ \\
\hline Eutrophication & kg N eq & Eutrophication & $\mathrm{kg} \mathrm{PO}_{4}{ }^{3-} \mathrm{eq}$ \\
\hline $\begin{array}{l}\text { Smog Creation } \\
\text { Potential }\end{array}$ & $\mathrm{kg} \mathrm{O}_{3} \mathrm{eq}$ & $\begin{array}{l}\text { Photochemical } \\
\text { Ozone Creation } \\
\text { Potential }\end{array}$ & $\mathrm{kg} \mathrm{C}_{2} \mathrm{H}_{4}$ eq \\
\hline
\end{tabular}

Table 48: Impact Category Characterization Factors from PCR, for B vs J Comparison

\section{Environmental Aspects that "will" be Included}

Use of material and energy resources:

- Use of non-renewable material resources

- Use of renewable material resources

- Use of non-renewable primary energy (units: megajoules):
o Fossil oil
o Natural gas
o Coal
o Uranium

- Use of renewable primary energy (units: megajoules):
o Hydropower
o Wind power
o Solar power
o Biomass

Use of water

Impact category indicator will include, but not be limited to, results for:

- Climate change. Emission of greenhouse gases.

- Destruction/depletion of ozone layer. Emission of ozone-depleting gases.

- Acidification of land and water sources. Emission of acidifying gases.

- Eutrophication. Emission of substances contributing to eutrophication potential.

- Formation of photochemical oxidants. Emission of gases that contribute to the creation of ground-level ozone.

Waste to disposal

- Non hazardous waste $(\mathrm{kg})$

- Hazardous waste $(\mathrm{kg})$

Waste to energy 
Both EPDs fail to meet the PCR's requirements set out in the section regarding the environmental impacts that "will" be included in every single EPD that uses this PCR. B only fails to declare the formation of tropospheric ozone (referred to as "smog creation potential" or "photochemical ozone creation potential" in the PCR), while $\mathrm{J}$ fails to mention this and others, such as use of materials, energy, and generation of waste. This specific downfall leads to the incomparability within these two EPDs, even though they follow the same PCR. The reason for this is clear: both EPDs did not follow the requirements of the PCR. However, it is hard to give a concrete reason as to why, other than present a few possibilities:

1. The authoring of the EPDs is of an insufficient level, as this very critical category is not followed according to the PCR.

2. The PCR is not clear enough for the author in emphasizing the categories that must be included.

3. The verification process of the EPDs is of an insufficient level, as these EPDs has been published with this critical category partially omitted, albeit at varying levels.

4. Or, a combination thereof.

For the second crucial category, cut-off rules, Table 51 shows the PCR's requirements alongside their application in both EPDs.

\section{PCR Requirements}

A process or activity that contributes no more than $2 \%$ of the total mass and $1 \%$ of the total energy use may be omitted from the inventory analysis, except that:

6.3.1 Omissions of any material flows that may have a relevant contribution to the selected impact categories of the products underlying the Environmental Declaration will be justified, if applicable, by a sensitivity analysis.

The sum of the excluded material flows must not exceed $5 \%$ of mass, energy or environmental relevance.

\section{B}

$\mathbf{J}$
Air emissions, water discharges and solid waste were allocated considering the production rates, all unit processes were No
mention. considered, thus no cut off principles were applied 
Cut-off rules are typically stated in EPDs in four differing ways: (1) the EPD will list the cut-off rules (similar to how it is written in the PCR Requirements column in Table 49 above) and declare whether or not they were applied; (2) the EPD will list the cut-off rules but not disclose if they were applied or not; (3) the EPD will disclose that no cut-off rules were applied without mentioning what the cut-off rules actually are (similar to B in Table 49); and (4) the EPD will have no mention of cut-off rules whatsoever (similar to $\mathrm{J}$ in Table 49). With a category that has this much bearing on comparability, it is concerning that there are these many ways to disclose the cut-off rules with neither option being comparable to another (situation-dependent).

The PCR's requirements for the cut-off rules to be applied in the LCA are very clear. B seems to acknowledge the cut-off rules exist and declares that they were not applied as part of the LCA process. However, what the cut-off rules exactly are is not known unless the user also reads the PCR. J does not mention them at all, so it is not known both what they are, and if they were applied. There are only two scenarios in which comparability can be assured regarding cut-off rules in EPDs: (1) both EPDs state cut-off rules were not applied (no bearing on whether the set of cut-off rules is exactly the same or not); or (2) one or both of the EPDs state cut-off rules were applied, and both list the same exact set of cut-off rules.

One current deficiency regarding EPDs, which has been discussed in academic literature (Modahl, Askham, Lyng, 2013) and has been reinforced by this exercise is the use of the terms "identical" and "equivalent" in ISO 14025 when referring to comparability. The standard requires items in an EPD to be "identical" or "equivalent" for comparison, but never defines what these terms exactly entail in the standard. This leaves it to the reader to determine what "identical" and "equivalent" both entail when comparing EPDs, which is problematic. In this thesis, items that were required to be "identical" and "equivalent" were judged with a logical and reasoned approach on a case-by-case basis, where "identical" items needed to be exactly the same (or converted to be exactly the same, i.e. in the case of functional units) and "equivalent" items needed to be moving towards the same end-goal with a similar method. The problem is that this particular approach could potentially differ from other practitioner's approaches due to a lack of definition in these terms.

Hunsager, Bach, and Breuer (2014) present an interesting discussion on verification. As discussed earlier in this section, it is possible problems may arise with the verification process of EPDs, given that some EPDs from the same PCR were incomparable according to the EPD Comparison Matrix. In ISO 14025 Section 8.1.1, program operators are required to use an "appropriate verification process", which could potentially allow for internal verification. It is only 
for EPDs that are aimed at consumers where third-party verification is required. However, these definitions also have inherent failures. EPDs meant for business-to-business communication (i.e. not third-party) can easily be publicly available and used comparatively in their product documentation. Additionally, there are some programs that offer LCA calculation and EPD verification services, where the program operator can act as both the LCA author and EPD verifier. It is not known if these are the reasons for the deficiencies regarding EPDs with the same PCR, but it is a grey area that is worth addressing in the standard.

There is another inherent problem with EPDs exacerbated by the uncertainty of verification. One of the obstacles designers will face at some point will be skepticism in the trustworthiness of the manufacturer's data. Whether or not this thought is founded in truth, there will always be cases when EPDs are being published from manufacturers that are unfamiliar to the project designers. The only document that could alleviate this lack of trust is the Project Report, which includes a detailed overview of the product's LCA. However, the user is never able to see the entire Project Report, and this is confidential as it contains trade secrets.

There are three steps that can, at the very least, alleviate some potential concerns designers have. First, there needs to be more LCA information given to consumers in EPDs, as it seems like the most important component for comparison. Second, if more information is not possible (i.e. providing more information may potentially expose trade secrets) there must be more clarity of information, transparent as possible without becoming a "fire sale" of trade secrets. A relatively large number of EPDs did not explicitly state the relevant data quality characteristics of the data used in the LCA as per ISO 14025, including "coverage, precision, completeness, representativeness, consistency, reproducibility, sources, and uncertainty". Doing so may help designers make better material sourcing decisions and become more informed about the products they use. Third, this information needs to be consistent across all EPDs.

\subsection{Issues Associated with Product Category Rules}

One of the main purposes of completing the PCR exercise was determining whether there was a possibility for large-scale comparison. If two PCRs were comparable based on the PCR Comparison Matrix, then hypothetically all EPD written under those two PCRs would be comparable to one another. However, no comparison could be seen after the exercise had been completed. Instead of drawing effort towards finding comparison within PCRs, it may be a more efficient and productive to find ways to identify poor PCRs that should be avoided, or conversely, develop a guideline to identify PCRs of good quality. In this discussion, the crucial 
categories used in the PCR Comparison Matrix that contributed to invalid comparison will be analyzed, classifying the good and bad within each category and the direction each category should move in the future.

The first aspect is the Functional/Declared Unit. Many of the invalid comparisons in the EPD Comparison Matrix in this category were due to two different natures of PCRs: one written for a generic, broad category (like construction products) and one for a specialized category (only for insulation products, or only for flooring products, etc). The generic, broad PCR cannot provide a specific functional or declared unit as there are too many products that fall under its scope. Instead, they mandate that declared units of mass (i.e. $1 \mathrm{~kg}$ ), volume (i.e. $1 \mathrm{~m}^{3}$ ), area (i.e. $1 \mathrm{~m}^{2}$ ), length (i.e. $1 \mathrm{~m}$ ), or an item (i.e. 1 brick) be used, instead of a specific unit, like in the case of insulation, a $1 \mathrm{~m}^{2}$ piece of insulation of appropriate thickness to provide a thermal resistance of $1 \mathrm{~m}^{2} \cdot \mathrm{K} / \mathrm{W}$. In this case, the EPD written to the generic PCR cannot be compared to the EPD written to the specific PCR, which is used most often within a product category. Generic PCRs are great for filling in gaps where PCRs do not exist for niche products or services. However, for popular items such as the product categories analyzed in this thesis (insulation, flooring, cladding), there is no need to use a generic PCR where specific PCRs exist and can ensure comparability in the functional/declared unit category.

Non-comparison in the System Boundary category was due to problems evaluating "identical" and "equivalent" requirements in ISO 14025, as already discussed in Section 9.1. All system boundaries across the PCRs analyzed were equivalent, all stages within this category had very similar components and goals, but were not identical (e.g. one PCR had an extra small substage that the other one did not) so they are then deemed incomparable. Similar to the issues associated with EPDs, definition on the terms "identical" and "equivalent" within an ISO standard would help clear up issues regarding PCRs.

The Data Quality Requirements in the PCR Comparison Matrix focused on the temporal, technological, and geographical requirements for the data used for the LCA. A surprisingly substantial amount of non-comparison in this category was due to PCRs omitting one or multiple of these requirements in their document. Other non-comparison was due to differences, mainly in the geographical requirement section, where PCRs are written for a specific country. For example, one PCR's geographical requirements include "Companies operating in Germany shall use the GaBi database for energy, transport and auxiliaries if intending to register EPDs in the German national EPD database... Companies operating outside of Germany may use other databases with transparent documentation required for mutual recognition, but shall not be 
included in the German national EPD database." It is hard to avoid these types of differences within PCRs and they should be expected. A best practice guideline for attempting to identify a good PCR would be to ensure that at the very least, temporal, technological, and geographical requirements are all included.

For a design practitioner, analyzing the cut-off rules and allocation rules may be too strenuous of an undertaking for minimal benefit in terms of comparison. Cut-off rules and allocation rules implemented in all PCRs analyzed in this research are all very similar. The non-comparison within this section came from small differences, such as $1 \%$ vs $2 \%$ for cut-off rules (discussed in Section 8.2.2), or verbiage differences within the text for allocation rules (again, relevant to the "identical" vs "equivalent" discussion throughout this thesis).

Differences in the last category (including impact categories and characterization factors) were due to PCRs choosing different characterization factors than others. For example, PCRs choosing to disclose acidification as $\mathrm{mol} \mathrm{H}+$ eq rather than the more popular $\mathrm{kg} \mathrm{SO}_{2}$ eq (see discussion on North America vs Europe in Section 8.2.1.7). Table 52 below shows a list of "best practice" impact categories and characterization factors when analyzing or picking PCRs to ensure the highest level of comparability in this category.

$\begin{array}{ll}\text { Impact Category } & \text { Characterization Factor } \\ \text { Global Warming Potential } & \mathrm{kg} \mathrm{CO}_{2} \mathrm{eq} \\ \text { Ozone Depletion Potential } & \mathrm{kg} \mathrm{CFC}-11 \mathrm{eq} \\ \text { Acidification } & \mathrm{kg} \mathrm{SO}_{2} \mathrm{eq} \\ \text { Eutrophication } & \mathrm{kg} \mathrm{PO}_{4}{ }^{3-} \mathrm{eq} \\ \text { Photochemical Ozone Creation Potential } & \mathrm{kg} \mathrm{C}_{2} \mathrm{H}_{4} \mathrm{eq} \\ \text { Use of Non-Renewable and Renewable Energy } & \mathrm{MJ} \\ \text { Use of Non-Renewable and Renewable Resources } & \mathrm{MJ} \\ \text { Freshwater Consumption } & \mathrm{m}^{3} \\ \text { Non-Hazardous and Hazardous Waste } & \mathrm{kg}\end{array}$

Table 51: Best Practice Impact Categories and Characterization Factors

PCRs that are currently being developed, or will be developed in the future should use the Guidance for Product Category Rule Development as a reference (see Section 2.5.1 for a full description of the document). Topics covered in the document include preparation for development, a review of the elements within a PCR, the PCR review process, publishing and maintaining a PCR, using a PCR, and best practices for developing and managing PCRs. 
Two specific areas to focus on that would help inform some level of harmonization in North America and globally are the alignment of PCRs and the creation of unified PCRs, both of which are recommended in the Guidance for Product Category Rule Development.

Alignment is a process that focuses on aligning data rules, LCA rules, and procedures to become consistent in PCRs across product categories. This ensures that EPDs that are created with strict adherence to an aligned PCR will be comparable. Essentially, this entails the coming together of program operators who have PCRs in a specific product category to establish "mutual intelligibility" between their own PCRs. This may include adapting or updating their current PCR to become similar enough to be comparable to one another as per the comparability requirements established in ISO 14025.

A unified PCR is one single overarching PCR that is developed from existing PCRs and aims to serve as the basis for all new EPD claims. It is usually based on existing PCRs of the same product category, is developed by the involvement of multiple program operators who have vested interest in alignment within their product category and PCRs, and supersedes all individual PCRs. The unified PCR can contain flexibility for regional or technological differences, the main strength of alignment.

Program operators in North America should work to either: a) begin the alignment process with others in the same product category to establish comparability between PCRs, or b) either adapt their PCR to consent to the unified PCR or plan the development of a unified PCR if one does not already exist.

\subsection{Use as an Evaluator of Harmonization}

One of the intended uses for the comparison matrices developed as part of this research is the ability to analyze the success of current harmonization techniques, and help test or build future harmonization schemes. The suitability for this can be seen through the comparisons of EPDs that follow EN 15804, a European standard intended to harmonize EPDs by acting as a PCR. The results of the comparisons of EPDs and PCRs, which both follow EN 15804, can provide insight to how well the harmonization technique is working, and the areas in which the standard, or the workflow from standard to published EPD/PCR, can be improved. Tables 53 and 54 show the results from these comparisons. 


\section{Product Category}

$\begin{array}{lll}\text { Insulation } & \text { Flooring } & \text { Cladding }\end{array}$

Total Comparisons (between

EPDs following EN 15804)

Total Valid Comparisons

Same Functional/Declared Unit

System Boundaries Separated or

Same

Impact Category Characterization

Factors

Mandatory Content for Comparison

Cut-Off Rules
28

$20(49 \%)$

$\mathbf{0}(0 \%)$

$\mathbf{0}(0 \%)$

$0(100 \%)$

$8(20 \%)$
28

$\begin{array}{lll}7(34 \%) & 0(33 \%) & 18(95 \%)\end{array}$

19

$0(0 \%)$

$0(0 \%)$

O $(0 \%)$

O (1\%)

7 (31\%)
$0(0 \%)$

$15(79 \%)$

Table 52: Invalidations in Comparisons, EPDs Both Following EN 15804

There are three main areas where poor definitions or misalignment invalidated comparisons between two EPDs both following EN 15804:

- Same Functional/Declared Unit: This is most prevalent in the insulation category, where most of the invalidations arose from comparisons where an EPD used a unit of $1 \mathrm{~m}^{2}$ of insulation material with a thickness that gives a design thermal resistance of $1 \mathrm{~m}^{2} \cdot \mathrm{K} / \mathrm{W}$, compared to an EPD which used $1 \mathrm{~m}^{3}$ of insulation material.

- Impact Category Characterization Factors: This largely resulted from EPDs not declaring environmental impacts in some of the impact categories named in this chart. In almost all cases, this is from one of the following impact categories: use of non-renewable energy, use of renewable energy, consumption of fresh water, hazardous waste, nonhazardous waste.

- Cut-Off Rules: These originated from small differences within the cut-off rules. In almost all cases, mass, and energy cut-off rules the same. Where differences occurred were in the environmental cut-off rules (e.g. "particular care should be taken to include material and energy flows with environmental impact" vs "should be included if it is less than $1 \%$ and environmentally relevant) and overall (i.e. total) cut-off rules of the entire LCA. In a few cases, cut-off rules were not disclosed in the EPD. 


\section{Product Category}

$\begin{array}{lll}\text { Insulation } & \text { Flooring } & \text { Cladding }\end{array}$

Total Comparisons (EN 15804)

Total Valid Comparisons

Product Category Definition and

Same

Functional/Declared Unit

System Boundaries

Data Quality Requirements

Cut-Off Rules

Allocation Rules

Life Cycle Impact Assessment

Requirements
6

0

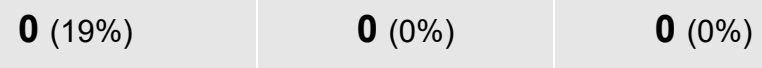

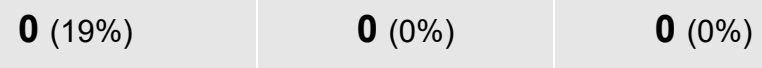

$\mathbf{0}(0 \%) \quad-\quad 0 \quad 0 \%)$

$4(60 \%) \quad-\quad 7(70 \%)$

$3(60 \%) \quad-\quad 4(40 \%)$

$3(90 \%) \quad-\quad 4(40 \%)$

$5(70 \%) \quad-\quad 4(40 \%)$

$6(100 \%) \quad-\quad 4(40 \%)$

$\begin{array}{lll}3(40 \%) & - & 0\end{array}$

Table 53: Invalidations in Comparisons, PCRs Both Following EN 15804

There are six key areas where poor definitions or misalignment invalidated comparisons between two PCRs both following EN 15804:

- Functional/Declared Unit: PCRs state the functional/declared unit in two different ways:

(1), to use a mass, volume, area, length, or an item, but does not specify what to use (e.g. open-ended PCRs for all construction products); (2), a specific area/volume of product (e.g. $1 \mathrm{~m}^{2}$ of flooring material). Invalidations arose when (1) was compared to (2), or (2) was compared to (2) with differing areas/volumes.

- System Boundaries: Invalidations in this category were a result from differing descriptions of what was required to be included in each life-cycle module. For example, PCR A requires the following: transportation from the production gate to the construction site; storage of products, including the provision of heating, cooling, humidity control, etc, and; transport of waste generated from the construction site. PCR B only requires transport from production gate to construction site. This is deemed an invalid comparison.

- Data Quality Requirements: Invalidations in this category were mostly due to PCRs not stating one or more of the temporal, technological, or geographic requirements. In the Insulation category, 6 of 6 comparisons stated temporal requirements, 1 of 6 stated technological requirements, and 2 of 6 stated geographic requirements. 
- Cut-Off Rules: These invalidations were due to small inconsistencies, similar to the phenomenon described in response to Table 52 above.

- Allocation Rules: Similar to cut-off rules, invalidations were due to small inconsistences. These mostly arose from requirements based on the country where the PCR originated (i.e. German requirements from one PCR that are not present in another American PCR).

- Life Cycle Impact Assessment Requirements: In the Insulation category, one PCR mandated that photochemical ozone creation, also referred to as formation of tropospheric ozone, be declared in ethyne $\left(\mathrm{C}_{2} \mathrm{H}_{2}\right)$. The other EPDs mandated it be declared in ethylene $\left(\mathrm{C}_{2} \mathrm{H}_{4}\right)$.

There are two reasons that could explain these invalidations. First, EN 15804 is possibly too lenient on allowing program operators to write specialized PCRs based on EN 15804 that don't fulfill its true purpose. This is seen in areas where provisions are added to PCRs for geographic reasons, such as allocation rules. This is also seen in areas where small changes are made, such as in cut-off rules, which may not have a large influence on the quantified environmental impacts. Second, this comparison methodology "expects too much" and there is a disconnect between how EN 15804 is supposed to work and ISO 14025's requirements for comparability. For example, EN 15804 states the functional/declared unit shall be in one of the following unit types: an item (e.g. 1 brick), a mass (e.g. 1 kg), a length (e.g. $1 \mathrm{~m}$ ), an area (e.g. $1 \mathrm{~m} 2$ ), or a volume (e.g. m3). Some PCRs written for all construction products reflect this statement, however, PCRs which follow EN 15804 but are written for specific product categories usually give a specific functional unit. This is a problem in product categories like insulation, where EPDs use functional units of unconvertable volumes (described following Table 52).

\subsection{Suggested Areas of Policy Improvement to Improve Comparability}

1. Consistent Functional Units: Most of the invalidations in regards to functional units arose from the use of broadly written PCRs for products that have PCRs written for the specific product category, which may be more suitable for use when authoring an EPD. Two steps can be taken to address this deficiency: one, mandate the adoption of specialized PCRs where appropriate in the industry, and two, improve the alignment of PCRs to create mutual intelligibility between specialized PCRs or unified PCRs to act as regional or global PCRs rather than national PCRs. 
2. Impact Category Characterization Factors: EN 15804 mandates that impact assessment is carried out for the following impact categories (e.g. global warming potential, ozone depletion) and resource use categories (e.g. fresh water consumption, non-renewable energy use, hazardous waste). However, some of these categories are not declared within some of the EPDs that followed EN 15804 analyzed in this research. This is due to inadequate authoring and verification processes. Improvements to policies, related to stricter guidelines for authoring and verification and the inclusion of specific impact categories and the use of consistent characterization factors, would be desirable in this respect.

3. Cut-Off and Allocation Rules: A large portion of EPD and PCR comparisons were excluded from comparison based on these two parameters. From a designer's perspective, it is not apparent if a difference between, for example, an energy exclusion of $2 \%$ vs $1 \%$ deserves to preclude a comparison, especially when cut-off rules are not applied in many instances for construction products. Additionally, it was found that cut-off rules were not discussed in many of the EPDs analyzed as part of this research, even though they are required to be identical for comparison as per ISO 14025. Similarly, a small geographic difference may not be enough to preclude a PCR comparison. It is not apparent how necessary it is for both these sets of rules to be identical, considering the potential low effect it has on the comparison. A reanalysis of the inclusion of these parameters as identical vs equivalent (in addition to definitions for identicalness and equivalency) should be taken into account in future versions of EPD standards, especially with the importance of harmonized comparisons as more manufacturers adopt and start producing EPDs. 


\section{Future Research}

EPD use is set to increase globally as part of its implementation into sustainable rating systems, including LEED® v4. This provides an opportunity for a greater level of research in regards to EPDs on construction projects than presently exists. Future research in this area has three lines of investigation, first to develop a greater understanding of their use on construction projects, second to enhance the comparison process, and third to develop techniques to aid harmonization or to assess harmonization efforts within the industry.

The first line of investigation is based on the fact that this thesis analyzed the only building possible; there has only been one LEED ${ }^{\circ}$ v4 building in Canada to have used EPDs as part of the design process. This rating system is currently at a relatively low level of adoption, but the date at which it must be used on new $L E E D \otimes$ projects will pass shortly after the time of publication (October 2016). As the use of EPDs on design projects increases, it would be useful for additional case studies to be completed to validate findings, correct the conclusions drawn, or identify other aspects of using EPDs as a design tool not found in this case study.

The second line of investigation would build on the comparison research presented in this thesis and create a more intuitive, automated experience for design practitioners. There have been previous attempts to compare PCRs and EPDs, and this thesis is the first instance in where the gap is attempting to be bridged between research and potential use in industry. However, a toolkit based heavily on user experience that can, for instance, automate conversions between characterization factors or break down environmental impacts into easy-to-use informative charts could potentially help increase the effectiveness EPDs have on a construction project.

The third line of investigation addresses the fact that harmonization is one of the key problems holding back EPDs and their use as comparative tools. This research does not focus strictly on harmonization techniques or principles, instead identifying key areas harmonization should address. Some areas of research include developing improved harmonization techniques, or ways that harmonization techniques can be evaluated. The toolkit developed as part of this thesis, in its current or a future version, can also help inform or identify whether harmonization techniques are successful, and can be adapted for future improvements and policy changes to EPDs. 


\section{Conclusions}

There are eight major conclusions that have been drawn from this research;

1. The effect EPDs have on the project timeline is a key concern for contractors, while the limited transparency of EPD development is a concern for designers. The integrative design process was critical to the success of the case study project, as it allowed for the early sourcing and purchasing of materials and comprehensive review of project specifications.

2. Having EPD credits within sustainable rating systems is an excellent incentive for manufacturers to create EPDs for their products and designers to implement the products in construction projects.

3. Life cycle inventory methodology, environmental indicators, and life cycle inventory background databases are the key areas required for harmonization within EPDs. These factors can be analyzed within a comparison tool similar to the one provided in Sections 4-7.

4. A methodology or tool to compare EPDs and PCRs is not only the preferred method to assess the comparability of two different environmental claims but can be an effective tool to assess the overall harmonization within entire product categories. Additionally, this can help identify areas for harmonization or evaluate different harmonization techniques.

5. There is a relatively high level of incomparability between EPDs with the same PCR based on the stipulations within international standards, despite this being a requirement of resources.

6. There is a greater level of comparability within product categories that have been investigating the environmental impacts of their products for a greater period of time, such as flooring.

7. There is a general lack of valid comparison between EPDs with different PCRs.

8. Comparability between PCRs is non-existent in the product categories analyzed. Most of this stems from either the use of broad PCRs (general construction products and services) as opposed to specific PCRs specialized for a specific product category (like insulation materials), or technical and regional differences, which are difficult to avoid. 


\section{Appendix A: Interview Questions}

This appendix includes the full question list that framed the semi-structured interviews. Each question set for each of the three interviewee groups is provided below

\section{Interview Questions for Project Team}

(Note to REB: only the questions (and not the possible answers) will be provided to participants in advance of the interview)

\section{For All Participants}

$<$ Review the consent form together>

Do you have any questions or concerns regarding this consent form?

Having reviewed this, do you consent to participate in this interview?

\section{For Client/Owner}

Thank you! This interview is intended to explore a series of topics related to Environmental Product Declarations, specifically their use in LEED\& v4, and your project experience on the CaGBC National Office.

1. What was your experience with environmental product declarations before CaGBC National?

2. Why did you request the use of environmental product declarations in your building? (open-ended question)

3. On a scale of 1 to 10 , with 1 being minimum, 4 being a little worse than average, 6 being a little better than average, and 10 maximum, how would you rate your:

a. Knowledge of EPDs

b. Understanding of LEED $®$ v4

4. After the use of environmental product declarations on this project, what are your thoughts on:

a. The degree in which EPDs are relied upon

b. The extent to which this reliance is appropriate

5. Would you insist on the use of environmental product declarations in your future work, specifically: (please explain)

a. LEED® projects?

b. Non-LEED $®$ projects? 
(Prompt) Thank participants for their time and offer to share the results of the survey and copy of the eventual article with them.

\section{For Project Designer/Architect}

Thank you! This interview is intended to explore a series of topics related to Environmental Product Declarations, specifically their use in LEED\& v4, and your project experience on the CaGBC National Office in Vancouver.

1. Did you decide to take on Material and Resources credit Building Product Disclosure and Optimization - Environmental Product Declarations?

a. If yes, what was your main motivation behind pursuing the credit?

i. Relatively easy credit to qualify

ii. High target of certification which required exploration in other credit categories

iii. Owner/client requests for sustainable materials

iv. Owner/client requests for disclosed materials

v. Any other motivations

b. If no, please explain why.

2. Was the credit obtained successfully?

3. What was your experience with environmental product declarations or material sourcing before CaGBC National?

a. If yes, what were the goals of the project in which you were part of?

b. What were the specific goals related to the use of EPDs on the project?

i. Influenced by the owner?

ii. Influenced by a rating system?

iii. Any other influences?

4. Did you conduct more material research, less, or about the same when using environmental product declarations?

a. Was this research ultimately helpful to the project outcome?

b. Was it deemed necessary for the LEED $®$ credit?

5. How would you rank your concerns with the following aspects of environmental product declarations, before and after CaGBC National? (Please rate as "very high", "high", “neutral”, "low", "very low", "unchanged") 
a. Variability between product category rules:

i. In general?

ii. Of a specific product category?

b. Variability between methodologies used to conduct the life cycle assessment of a product?

c. Availability of Type III Environmental Product Declarations?

i. In general?

ii. From small- to medium-sized manufacturers?

6. On a scale of 1 to 10 , with 1 being not stringent at all, 4 being a little worse than average, 5 being a little better than average, and 10 being very stringent, how stringent was your design team in discovering the origins of the environmental product declaration? Specifically:

a. Ensuring products have the same product category rules for comparisons?

b. Generic or specific data for Life Cycle Assessments?

C. Third party verification?

7. Did you find that environmental product declarations helped your team make informed decisions on which products to use?

a. Was there added depth in the discussions of which products were chosen?

b. Did this ultimately help or hinder the experience of sourcing materials and products?

8. Do you feel comfortable enough to use materials with environmental product declarations in your future work?

9. After the use of environmental product declarations on this project, what are your thoughts on:

a. The degree in which EPDs are relied upon

b. The extent to which this reliance is appropriate

10. Would you insist on the use of environmental product declarations in your future work, specifically: (please explain)

a. LEED尺 projects?

b. Non-LEED® projects?

11. Do you have any other thoughts on EPDs not mentioned in any of the above questions? (open-ended) 
(Prompt) Thank participants for their time and offer to share the results of the survey and copy of the eventual article with them.

\section{For Contractor}

Thank you! This interview is intended to explore a series of topics related to materials which have associated Environmental Product Declarations, specifically your experience with the materials used on (insert project title here).

1. What was your experience with environmental product declarations before CaGBC National?

2. Did the use of materials with environmental product declarations have any bearing on the timeline of the project?

3. Did the use of materials with environmental product declarations have any bearing on the project cost?

4. Were there any products with declarations which had unusual specification requirements that were atypical?

5. How have specifications changed before EPDs having a role in the project and after?

6. After the use of environmental product declarations on this project, what are your thoughts on:

a. The degree in which EPDs are relied upon

b. The extent to which this reliance is appropriate

7. Would you insist on the use of environmental product declarations in your future work, specifically: (please explain)

a. LEED® projects?

b. Non-LEED $®$ projects?

(Prompt) Thank participants for their time and offer to share the results of the survey and copy of the eventual article with them. 


\section{Appendix B: Comparison Matrices, Templates, and Documents Used Within}

Included in this appendix is:

1. The completed EPD and PCR Comparison Matrices (EPD.PCR_Comparison_Matrices_2016.08.11.xlsx)
a. All EPDs used in the EPD Comparison Matrix
b. All PCRs used in the PCR Comparison Matrix

2. The blank Simplified EPD Comparison Template (Simplified_EPD_Comparison_Template_2016.08.11.xlsx)

All documents are provided on the compact disc affixed to the inside back cover of this thesis. 


\section{References}

Ball, J. (2002). Can ISO 14000 and eco-labelling turn the construction industry green? Building and Environment, 37(4), 421-428.

Bergman, R., \& Taylor, A. (2011). EPD - Environmental product declarations for wood products - An application of life cycle information about forest products. Forest Products Journal, 61(3), 192-201.

Buildings Performance Institute Europe. Europe's Buildings Under the Microscope: A countryby-country review of the energy performance of buildings. 2011.

Del Borghi, A. (2012). LCA and communication: Environmental Product Declaration. International Journal of Life Cycle Assessment, 18(2), 293-295.

DIALOG, LEED® v4 Case Study: CaGBC Vancouver Office Project Design, 2015. Webinar presentation.

EN 15804:2012+A1:2013, Sustainability of construction works - Environmental product declarations - Core rules for the product category of construction products, British Standards Institute, 2013.

Environment and Climate Change Canada, Canadian Environmental Sustainability Indicators Greenhouse Gas Emissions. 2016.

Erlandsson, M., Ekvall, T., Jelse, K., Lindfors, L., Gustavsson, M., Karlsson, P., ... Zetterberg, L. (2013). Robust LCA: PCR guide for construction products and works - Specifications to and evaluation of EN 15804 Bibliographic data. Stockholm.

Fet, A. M., Skaar, C., \& Magerholm Fet, A. (2006). Eco-labeling, Product Category Rules and Certification Procedures Based on ISO 14025 Requirements. The International Journal of Life Cycle Assessment, 11(1), 49-54.

Fet, A. M., Skaar, C., \& Michelsen, O. (2009). Product category rules and environmental product declarations as tools to promote sustainable products: Experiences from a case study of furniture production. Clean Technologies and Environmental Policy, 11(2), 201-207.

Frischknecht, R., Wyss, F., Knöpfel, S. B., \& Stolz, P. (2015). Life cycle assessment in the building sector: analytical tools, environmental information and labels. The International Journal of Life Cycle Assessment, 421-425. Guidance for Product Category Rule Development. (2013). Ingwersen, W., Subramanian, V., editors. The Product Category Rule Guidance Development Initiative. Version 1.0. http://www.pcrguidance.org

Haapio, A., Viitaniemi, P. (2008). A critical review of building environmental assessment tools. Environmental Impact Assessment Review, 28(7), 462-482.

Hunsager, E. A., Bach, M., \& Breuer, L. (2014). An institutional analysis of EPD programs and a global PCR registry. The International Journal of Life Cycle Assessment, 19(4), 786-795. 
IHS, Global construction outlook, IHS Economics, 2013. Web.

Ingwersen, W. W., \& Stevenson, M. J. (2012). Can we compare the environmental performance of this product to that one? An update on the development of product category rules and future challenges toward alignment. Journal of Cleaner Production, 24, 102-108.

Ingwersen, W. W., \& Subramanian, V. (2014). Guidance for product category rule development: process, outcome, and next steps. The International Journal of Life Cycle Assessment, 19(3), 532-537.

Innovation, Science and Economic Development Canada. Corporate Social Responsibility: SME Sustainability Roadmap. 2015. Accessed via: https://www.ic.gc.ca/eic/site/csrrse.nsf/eng/rs00585.html.

ISO 14021:1999, Environmental labels and declarations - Self-declared environmental claims (Type II environmental labelling), International Organization for Standardization, 1999.

ISO 14024:1999, Environmental labels and declarations - Type I environmental labelling Principles and procedures, International Organization for Standardization, 1999.

ISO 14025:2006, Environmental labels and declarations - Type III environmental declarations Principles and procedures, International Organization for Standardization, 2006.

ISO 14044:2006, Environmental management - Life cycle assessment - Requirements and guidelines, International Organization for Standardization, 2006.

ISO 21930:2007, Sustainability in building construction - Environmental declaration of building products, International Organization for Standardization, 2006.

ISO/DIS 21930, Sustainability in buildings and civil engineering works - Core rules used for environmental declaration of construction products and services used in any type of construction works, International Organization for Standardization, N.p.

Jönsson, Å. (2000). Tools and methods for environmental assessment of building productsmethodological analysis of six selected approaches. Building and Environment, 35(3), 223238.

LEED® Reference Guide for Building Design and Construction, U.S. Green Building Council, 2014, pp. 513-523.

Levine, M., D. Ürge-Vorsatz, K. Blok, L. Geng, D. Harvey, S. Lang, G. Levermore, A. Mongameli Mehlwana, S. Mirasgedis, A. Novikova, J. Rilling, H. Yoshino, 2007: Residential and commercial buildings. In Climate Change 2007: Mitigation. Contribution of Working Group III to the Fourth Assessment Report of the Intergovernmental Panel on Climate Change [B. Metz, O.R. Davidson, P.R. Bosch, R. Dave, L.A. Meyer (eds)], Cambridge University Press, Cambridge, United Kingdom and New York, NY, USA.

Means, P., Guggemos, A. (2015) Framework for the Life Cycle Assessment (LCA) based environmental decision making during the conceptual design phase for commercial buildings. International Conference on Sustainable Design, Engineering and Construction, 
$118,802-812$.

Minkov, N., Schneider, L., Lehmann, A., \& Finkbeiner, M. (2015). Type III Environmental Declaration Programmes and harmonization of product category rules: status quo and practical challenges. Journal of Cleaner Production, 94, 235-246.

Modahl, I. S., Askham, C., Lyng, K. A., Skjerve-Nielssen, C., \& Nereng, G. (2013). Comparison of two versions of an EPD, using generic and specific data for the foreground system, and some methodological implications. International Journal of Life Cycle Assessment, 18(1), 241-251.

O'Conner, J., Meil, J., Baer, S., Koffler, C. (2012) LCA in construction: status, impact, and limitations. Ottawa: Athena Sustainable Materials Institute.

Ortiz, O., Castells, F., Sonnemann, G. (2008) Sustainability in the construction industry: A review of recent developments based on LCA. Construction and Building Materials, 23(1), 28-39.

PD CEN/TR 15941, Sustainability of construction works - Environmental product declarations Methodology for selection and use of generic data, British Standards Institution, 2010.

Subramanian, V., Ingwersen, W., Hensler, C. (2011). PCR Comparison Template. Unpublished draft.

Subramanian, V., Ingwersen, W. (2012). Comparing product category rules from different programs: Learned outcomes towards global alignment. International Journal of Life Cycle Assessment, 12(7), 892-903.

Zackrisson, M., Rocha, C., Christiansen, K., \& Jarnehammar, A. (2008). Stepwise environmental product declarations: ten SME case studies. Journal of Cleaner Production, 16(17), 1872-1886. 